\title{
Evolution of active galactic nuclei
}

\author{
A. Merloni ${ }^{*}, 1$ and S. Heinz ${ }^{\dagger, 2}$ \\ ${ }^{1}$ Max-Planck-Institut für Extraterrestrische Physik, Giessenbachstr., D-85741, Garching, \\ Germany \\ ${ }^{2}$ Astronomy Department, University of Wisconsin-Madison, Madison, WI 53706
}

April 20, 2012

\begin{abstract}
Supermassive black holes (SMBH) lurk in the nuclei of most massive galaxies, perhaps in all of them. The tight observed scaling relations between SMBH masses and structural properties of their host spheroids likely indicate that the processes fostering the growth of both components are physically linked, despite the many orders of magnitude difference in their physical size. This chapter discusses how we constrain the evolution of SMBH, probed by their actively growing phases, when they shine as active galactic nuclei (AGN) with luminosities often in excess of that of the entire stellar population of their host galaxies. Following loosely the chronological developments of the field, we begin by discussing early evolutionary studies, when AGN observed at various wavelengths represented beacons of light probing the most distant reaches of the universe and were used as tracers of the large scale structure ("Cosmography"). This early study turned into a more mundane enterprise of AGN "Demography", once it was realized that the strong evolution (in luminosity, number density) of the AGN population hindered any attempt to derive cosmological parameters from AGN observations directly. Following a discussion of the state of the art in the study of AGN luminosity functions, we move on to discuss the "modern" view of AGN evolution, one in which a bigger emphasis is given to the physical relationships between the population of growing black holes and their environment ("Cosmology"). This includes observational and theoretical efforts aimed at constraining and understanding the evolution of scaling relations, as well as the resulting limits on the evolution of the SMBH mass function. Physical models of AGN feedback and the ongoing efforts to isolate them observationally are discussed next. Finally, we touch upon the problem of when and how the first black holes formed and the role of black holes in the high-redshift universe.
\end{abstract}

\section{Index Terms}

Active Galactic Nuclei (AGN), Quasars, Radio Galaxies, Supermassive Black Holes, Eddington Luminosity, Accretion, Jets, Scaling relations, AGN Number Counts, AGN Luminosity Functions, AGN Clustering, Cosmic X-ray Background, Black Hole Mass Function, AGN Feedback, Galaxy Evolution, Black Hole Formation

\footnotetext{
*am@mpe.mpg.de

†heinzs@astro.wisc.edu
} 


\section{Keywords}

\section{List of Abbreviations}

AGN: Active Galactic Nucleus

AU: Astronomical Unit

BAL: Broad Absorption Line

BHAR: Black Hole Accretion Rate

CSS: Compact Steep Spectrum

CXRB: Cosmic X-ray Background

DM: Dark Matter

FR I/II: Fanaroff-Riley class I/II

GPS: Gigahertz Peak Spectrum

ICM: Intra-Cluster Medium

IGM: Intra-Group Medium

LDDE: Luminosity Dependent Density Evolution

LADE: Luminosity And Density Evolution

LF: Luminosity Function

LLAGN: Low Luminosity Active Galactic Nuclei

PLE: Pure Luminosity Evolution

PDE: Pure Density Evolution

QSO: Quasi-Stellar Object

SED: Spectral Energy Distribution

SFR: Star Formation Rate

SMBH: Super-Massive Black Hole

\section{A historical perspective on AGN research}

The study of astrophysical black holes, as it has developed over the last five decades, is driven by three main rationales and goals. Because the mere existence of black holes is the most far-reaching implication of the theory of General Relativity (together with the Big Bang cosmological theory), they can first of all be used to test theories of gravitation in the strong field regime. Secondly, astrophysical black holes are revealed to us through emission processes taking place in accretion flows and relativistic jets, both originating in the black hole's deep potential well, and they offer a unique opportunity of studying interesting and complex astrophysical problems, involving extreme physical conditions, relativistic magnetohydrodynamics, and radiative effects. Thirdly, black hole formation and evolution might play an important role in a broader cosmological context, affecting the formation and the evolution of the structures they live in, such as galaxies, groups and clusters.

During the first golden ag $₫ 1$ of black hole astrophysics, efforts were focused on finding proof of the existence of black holes and to define their basic interactions with the environment (accretion

\footnotetext{
${ }^{1}$ This definition was introduced by the Caltech graduate Bill Press (Thorne, 1994) to identify the years between the early '60s and the early '70s.
} 


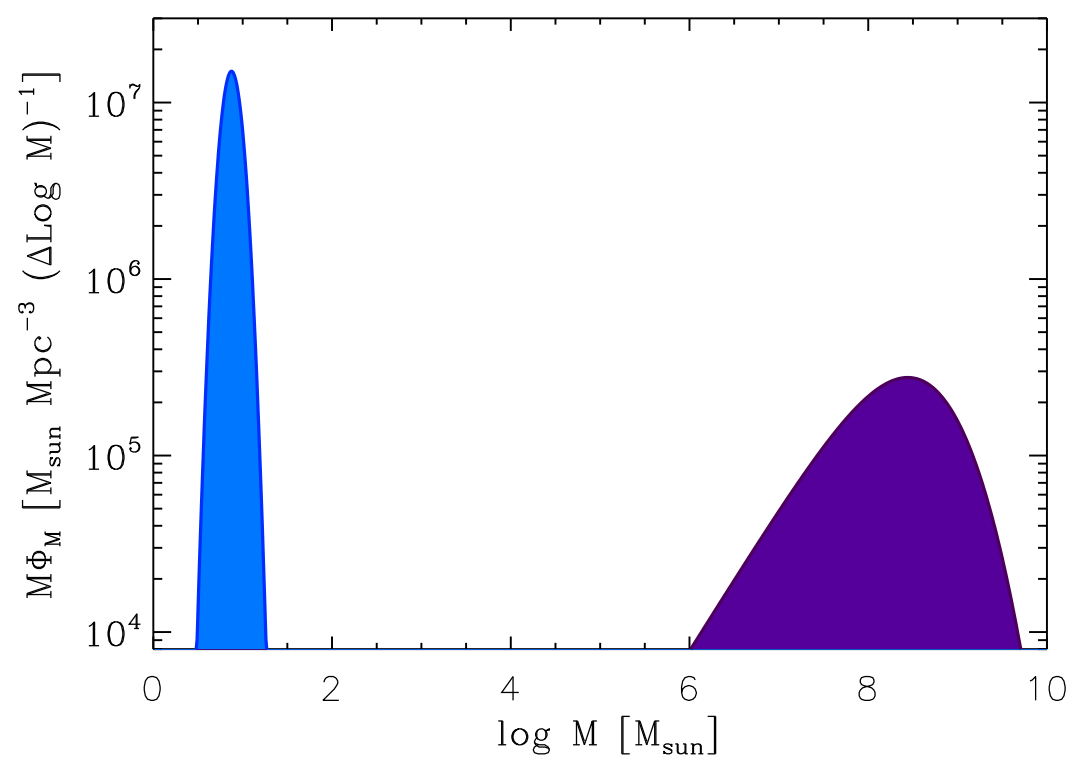

Figure 1: The local black hole mass function, plotted as $M \times \phi_{M}$ in order to highlight the location and height of the two main peaks in the distribution. The stellar mass black hole peak has been drawn assuming a log-normal distribution with mean mass equal to 5 solar masses, width of 0.1 dex and a normalization yielding a density of about $1.1 \times 10^{7} M_{\odot} \mathrm{Mpc}^{-3}$ (Fukugita \& Peebles, 2004 ), which is about $7 \times 10^{-5}$ times the critical density of the universe. The supermassive black hole peak, instead, contributes to an overall density of about $4.2 \times 10^{5} \mathrm{M}_{\odot} \mathrm{Mpc}^{-3}$, or a fraction only $2.7 \times 10^{-6}$ of the critical density (see section 3.1 for details).

and relativistic jet theory). Such goals only touched on the first two of the rationales listed above. The history of the development of black hole physics (both theoretical and observational) in these years has been beautifully laid out by Kip Thorne in his book "Black Holes and time warps: Einstein's outrageous legacy" (Thorne, 1994), where the reader can find a more complete set of references and biographical notes, together with the historical accounts presented elsewhere in this volume (see E. Perlman: "Active galactic nuclei").

Beginning at about the turn of the 21st century, black hole astrophysicists have acknowledged the relevance of their subject of study for a broader community of cosmologists and extragalactic astronomers, thanks to the multiple lines of evidence pointing towards a fundamental role played by black holes in galaxy evolution.

In fact, black holes in the local universe come in two main families according to their size, as recognized by the strongly bi-modal distribution of the local black hole mass function (see Fig. 1). While the height, width and exact mass scale of the stellar mass peak should be understood as a by-product of stellar (and binary) evolution, and of the physical processes that make supernovae and gamma-ray bursts explode, the supermassive black hole peak in this distribution is the outcome of the cosmological growth of structures and of the evolution of accretion in the nuclei of galaxies, likely modulated by the mergers these nuclear black holes will experience as a result of the hierarchical galaxy-galaxy coalescences. 
This picture of the local demographics of black holes has been made possible by the discovery of tight scaling relations between the central black hole mass and various properties of their host spheroids (velocity dispersion, $\sigma_{*}$, stellar mass, $M_{*}$, luminosity, etc.) that characterize the structure of nearby inactive galaxies (Magorrian et al. 1998; Gebhardt et al. 2000; Ferrarese \& Merritt 2000; Häring \& Rix 2004; Gültekin et al. 2009, see Figure 21).

These correlations are a result of the search for local QSO relics via the study of their dynamical influence on the surrounding stars and gas made possible by the launch of the Hubble Space Telescope. They have revolutionized the way we understand the physical link between the evolution of galaxies and active galactic nuclei (AGN2).

In addition, it is now understood that supermassive black hole (SMBH) growth is due mainly to radiatively efficient accretion over cosmological times, taking place during active phases (see $\S 3.1$ below). This, together with the understanding of a near universal presence of black holes in galactic centers has led to the suggestion that most, if not all, galaxies went through a phase of nuclear activity in the past, during which a strong physical coupling (generally termed "feedback") might have established a long-lasting link between host and black hole properties.

Such a renewed interest for AGN in a cosmological context requires a good understanding of the evolutionary properties of this class of objects. The fact that AGN and quasars were a strongly evolving class of astronomical sources became evident very soon after their discovery, as we will discuss at length in the following sections. Nonetheless, the appreciation that such an evolution could not only mirror, but also influence that of galaxies, groups and clusters, only became commonplace after the discovery of the above mentioned scaling relations.

In this chapter we will focus on the current knowledge of AGN evolution. Following loosely the chronological developments of the field, we will begin by discussing the "first generation" of AGN evolutionary studies ( $(2)$, during which AGN observed at various wavelengths represented beacons of light probing the most distant reaches of the universe and were used as tracers of the structures themselves.

This short-lived epoch of AGN "Cosmography" quickly gave way to a more mundane enterprise of AGN "Demography", once it was realized that the strong evolution (in luminosity, number density, etc.) of the AGN population hindered any serious attempt to derive cosmological parameters from AGN observations directly. The attention then moved to the study of the evolution of active galactic nuclei by means of determinations of their luminosity functions. An update on the most recent works on the luminosity functions of AGN selected in different ways from different electromagnetic bands will also be given in section 2 , which will be closed by a brief discussion of AGN clustering as a natural complementary cosmographic tool ( $\$ 2.2$ ).

We will then move to discuss the "modern" view of AGN evolution, one in which a bigger emphasis is given to the physical relationships between the population of growing black holes and their environments. We call this the "Cosmology" phase of AGN studies, to highlight the close link between these subject areas that has been established in recent years. We will first discuss observational and theoretical efforts aimed at constraining and understanding the evolution of the scaling relations, as well as the resulting limits on the evolution of the SMBH mass function

\footnotetext{
${ }^{2}$ In this chapter, we will use both the term AGN and QSO/quasar to indicate actively growing supermassive black holes, implying no real physical distinction between the two, apart from one based on the total emitted luminosity: while AGN can be used for any objects, QSO/quasar usually identify those with bolometric luminosity $\log L_{\mathrm{bol}}>46$ in cgs units.
} 


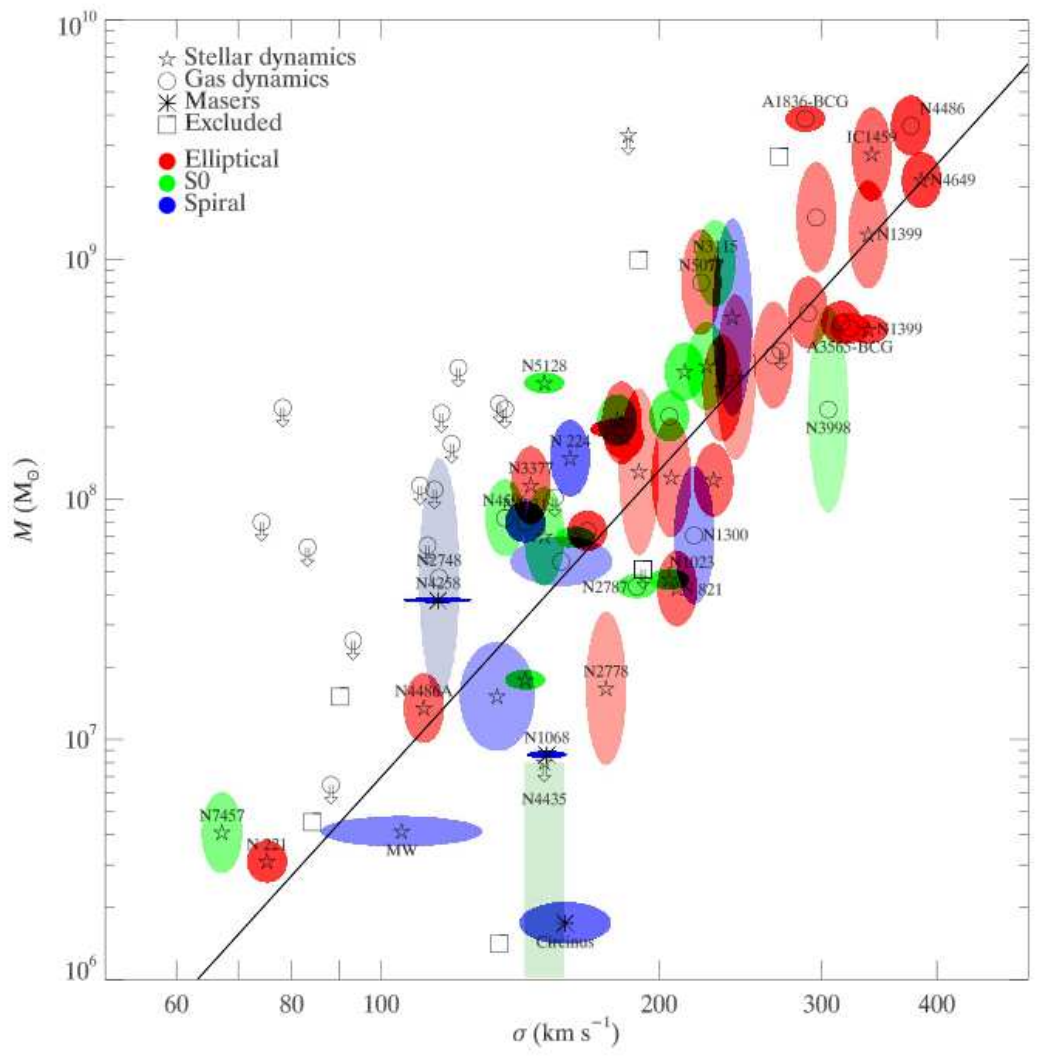

Figure 2: The $M_{\mathrm{BH}}-\sigma_{*}$ relation for galaxies with dynamical $\mathrm{BH}$ mass measurements. The symbols indicate the method of BH mass measurement: stellar dynamical (pentagrams), gas dynamical (circles), masers (asterisks). Arrows indicate $3 \sigma$ confidence upper limits to the $\mathrm{BH}$ mass. The color of the error ellipse indicates the Hubble type of the host galaxy: elliptical (red), S0 (green), and spiral (blue). The saturation of the colors in the error ellipses or boxes is inversely proportional to the area of the ellipse or box. Squares are galaxies not included in the fit. This is shown as a solid line for the best fit relation to the full sample: $M_{\mathrm{BH}}=$ $10^{8.12} M_{\odot}\left(\sigma_{*} / 200 \mathrm{~km} \mathrm{~s}^{-1}\right)^{4.24}$ (adopted from Gültekin et al. 2009). 
(§ 3), and we will then present physical models of AGN feedback and the ongoing efforts to isolate them observationally in section 4 .

Finally, in the last section of this chapter $(\S[$ ) we will touch upon the problem of when and how the first black holes form and the role of black holes in the high redshift universe.

Before all this, however, a small diversion is in place. To be consistent, the very notion of an evolutionary study of any particular class of objects, not only in astrophysics, requires a definition of the non-evolving substratus that allows us to first identify an object as a member of the class, the evolution of which one wishes to study. To make just a simple example drawn from astronomical research, the evolution of galaxies is very much complicated by the never-ending morphological and photometric transformation of the different populations, so that a non-trivial element of any such study is the identification of progenitors and offspring along the Hubble sequence (see the Chapter R. Buta: "Galaxy Morphology" in this volume).

\subsection{Redshift evolution in AGN Spectral Energy Distributions}

The overall spectral energy distribution (SED) of AGN extends over many decades in frequency, and is the result of a number of different emission processes acting at different physical scales. We refer the reader to Chapter "Active Galactic Nuclei" by E. Perlman in this book for a thorough discussion of these processes and of the main characteristic of AGN SED.

The observational appearance of an active galactic nucleus is determined not only by its intrinsic emission properties, but also by the nature, amount, dynamical and kinematic state of any intervening material along the line of sight. AGN obscuration is a crucial factor for our general understanding of the AGN phenomenon. For example, in the traditional unificationby-orientation schemes different classes of AGN are explained on the basis of the line-of-sight orientation with respect to the axis of rotational symmetry of the system (see e.g. Antonucci 1993; Urry \& Padovani 1995, and references therein).

At odds with such simple schemes, evidence for a variation of the fraction of obscured AGN as a function of luminosity has been mounting recently (Ueda et al., 2003; Steffen et al., 2003; Simpson, 2005; Hasinger, 2008). The fraction of absorbed AGN, defined in different and often independent ways, appears to be lower at higher nuclear luminosities. This might be considered a signature of AGN feedback (in the "quasar" mode, see $\S 4.6$ below), in that powerful sources are able to clean up their immediate gaseous environments, responsible for the nuclear obscuration, more efficiently.

After accounting for such a clear luminosity-dependence, it is currently unclear whether the overall incidence of obscuration and extinction in the nuclear regions of a galaxy evolve with redshift. This would be expected if, for example, nuclear obscuration were causally linked to the overall amount of gas within galaxies, a quantity that increases obviously with redshift.

What we are interested in here, however, is any possible evidence of redshift evolution (or lack thereof) of the intrinsic AGN spectral properties, i.e. those characterizing the emission processes associated with the major mode of radiative energy release.

$\mathrm{X}$-ray emission is ubiquitous in AGN, and is a very effective way for selecting accreting black holes due both to the minimal contamination of star-forming processes and due to the decreasing importance of obscuration at increasing X-ray energies. Unfortunately, the exact mechanism responsible for AGN X-ray emission and its physical location are not fully understood yet (see Chapter "Active Galactic Nuclei" by E. Perlman in this book). Still, as a very general diagnostic, 

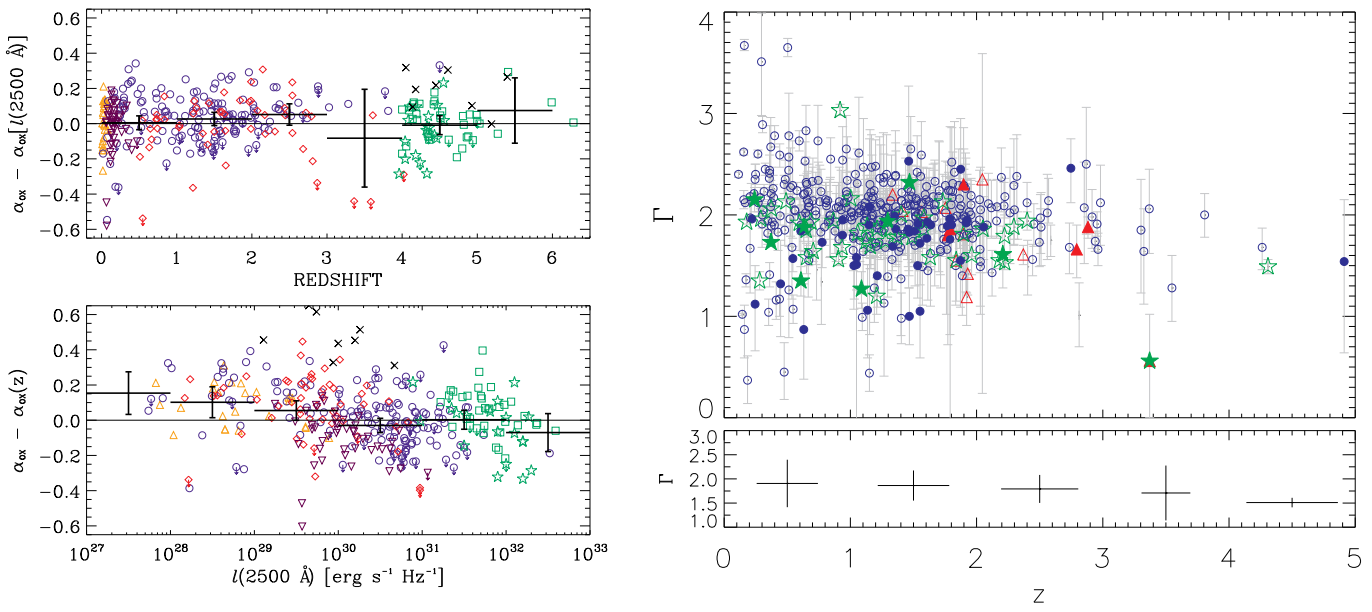

Figure 3: Left: $\alpha_{\text {ox }}$ residuals as a function of redshift (top panel) and luminosity density at $2500 \AA$ (bottom panel). The overlaid error bars denote the mean and the $3 \sigma$ standard deviation of the mean of the residuals. Limits are denoted with arrows. The systematic residuals in the lower plot indicate that $\alpha_{\text {ox }}$ cannot be dependent on redshift alone (adopted from Steffen et al. 2006); Right: X-ray photon index $(\Gamma)$ vs. redshift $z$. Blue circles represent radio quiet, non-BAL (Broad Absorption Line) quasars, green stars represent radio loud quasars, and red triangles represent BAL quasars. The bottom plot shows the weighted mean $\Gamma$ values for bins of width $\Delta z=1$. No clear sign of evolution in the average X-ray spectral slope of AGN is detected over more than $90 \%$ of the age of the universe (from Young et al. 2009).

the "X-ray loudness", usually characterized by the $\alpha_{\text {ox }}$ parameter, i.e. the slope of the spectrum between $2500 \AA=5 \mathrm{eV}$ and $2 \mathrm{keV}: \alpha_{\mathrm{ox}}=0.3838 \log \left(F_{2 \mathrm{keV}} / F_{2500}\right)$ can be used to characterize the fraction of bolometric light carried away by high-energy X-ray photons. Recent studies of large samples of both X-ray and optical selected AGN have clearly demonstrated that $\alpha_{\mathrm{ox}}$ is itself a function of UV luminosity (see e.g. Steffen et al. 2006; Young et al. 2009). However, no redshift evolution can be discerned in the data, as shown in the left panel of Figure 3 .

Moreover, large collecting-area X-ray telescopes allow a more precise determination of the Xray spectra of AGN, which are usually characterized by a power-law, upon which emission lines and absorption features are superimposed. Up to the highest redshift where reliable spectral analysis of AGN can be performed, no clear sign of evolution in the X-ray spectral slope $\Gamma$ has been detected (see the right panel of Figure 3).

Similarly, while the narrow iron $\mathrm{K} \alpha$ emission line, the most prominent feature in AGN X-ray spectra, is clearly dependent on luminosity (the so-called Iwasawa-Taniguchi effect Iwasawa \& Taniguchi 1993), it shows no sign of evolution in its equivalent width with redshift, at least up to $z \simeq 1.2$ (Figure 4. Chaudhary et al. 2010, and references therein).

Even more surprising is the lack of evolution in the optical emission line properties of QSOs. The metallicities implied by the relative strength of broad emission lines do not show any significant redshift evolution: They are solar or super-solar, even in the highest redshift QSOs known (see e.g. Hamann \& Ferland 1992), in contrast with the strong evolution of the metallicity in star forming galaxies. 


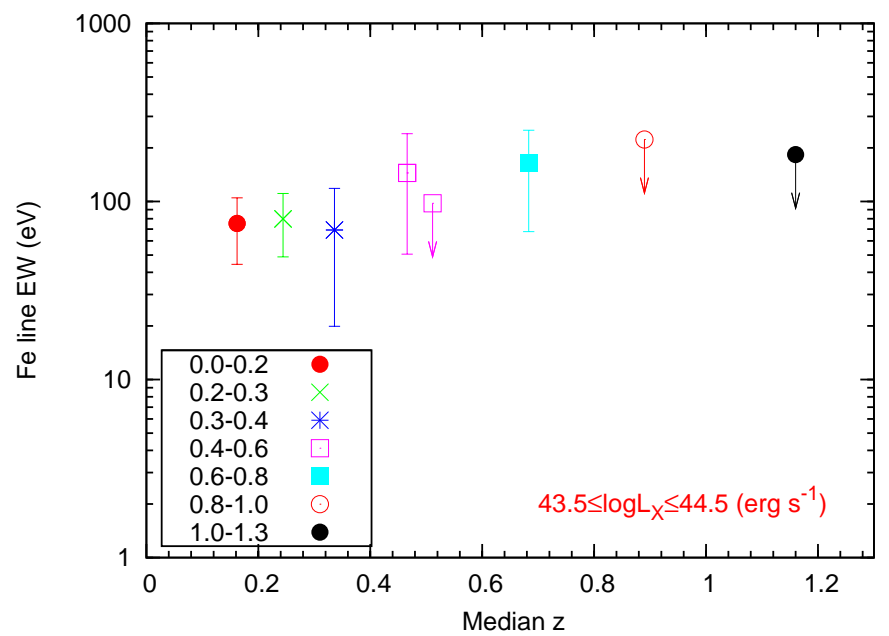

Figure 4: Rest frame equivalent width of the narrow Iron $\mathrm{K} \alpha$ emission line observed in the average spectrum of AGN as a function of redshift. Only objects in a fixed (2-10 keV) luminosity range $10^{43.5}<L_{2-10}<10^{44.5} \mathrm{ergs} / \mathrm{s}$ were considered. From Chaudhary et al. 2010.

A pictorial view of this surprising uniformity is shown in the left panel of figure 5, where the raw spectra from $\sim 17,000$ QSOs extracted from the Sloan Digital Sky Survey (SDSS) are plotted next to each other in a sequence of increasing redshift from bottom to top. The right panel of Fig. 5 shows a direct comparison of stacked QSO spectra in three redshift intervals (Juarez et al., 2009), where it is clear that the flux ratios among the most prominent lines stay almost constant up to the highest redshift probed.

Summarizing, there exists a remarkably uniform set of spectral characteristics that defines active nuclei at all epochs in the history if the universe, at least if we consider objects of a fixed total (bolometric) luminosity. The simplest explanation is that the emission properties from AGN, i.e., those which are (in most cases, at least) set by physical processes taking place within the gravitational sphere of influence of the central black hole, are essentially dictated by the gas and plasma dynamics there, where the central object's gravity dominates. We should then expect them to be relatively insensitive to the cosmological epoch, which instead greatly affects the properties of matter (density, temperature, ionization state, etc.) at the generic outer boundary, i.e., right outside the SMBH gravitational sphere of influence.

\section{Cosmography and Demography}

Accreting supermassive black holes have long been the lighthouses of our observable universe, holding the record of the most distant object known for more than four decades. As such, they have played a key role in the early phases of cosmological investigations.

Already in 1955 the second Cambridge catalog (2C) of unresolved radio sources (the so-called 'radio stars') observed at $81 \mathrm{MHz}$ (3.7 meters) had shown both a remarkable uniformity in the distribution of objects in the sky and an increase in the cumulative number counts (see below) that allowed Ryle \& Scheuer (1955) to unambiguously demonstrate not only their extragalactic 

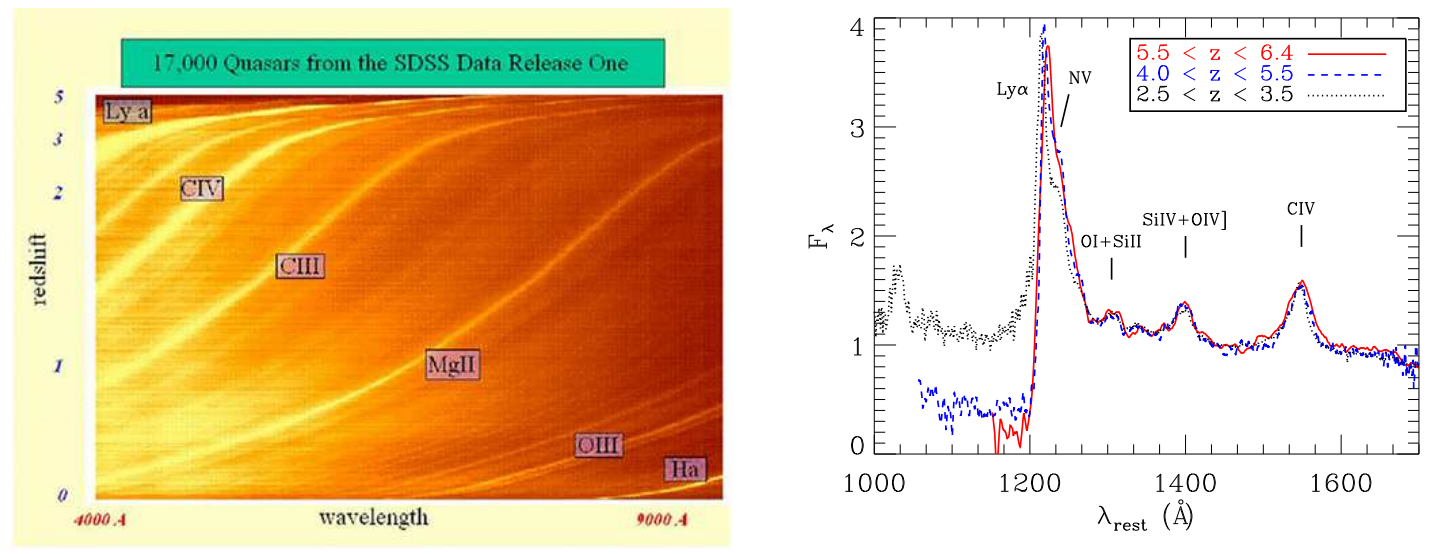

Figure 5: Left: Spectra of 17,000 QSOs from SDSS. Notice the large degree of uniformity in the relative intensity of the main emission features (courtesy of X. Fan); Right Stacked spectra of quasars in different redshift bins. Note that the relative intensity of the metal lines (and in particular the (SiIV+OIV])/CIV ratio) remains constant over the wide redshift interval 2.5jzi 6.4 , indicating that the metallicity in the observed quasars does not evolve with redshift. From Juarez et al. (2009).

origin, but also that the bulk of the source should lie at distances larger than a few tens of Mpc, i.e. well beyond the edge of the optically observable universe at the time.

The dispute over the exact shape of the radio source number count distribution that ensued soon afterwards became a key part of the debate between "steady state" and "evolutionary" models of the universe, lending strong support against stationary universe models (see Figure 6). Just two years after the discovery of quasars (Schmidt, 1963) with their exceedingly large redshifts, A. Sandage wrote: "The objects would seem to be of major importance in the solution of the cosmological problem. They can be found at great distances because of their high luminosity. Studies of the [number counts] curves using lquasars] should eventually provide a crucial test of various cosmological models" (Sandage, 1965). Similar hopes were expressed by Longair the following year (Longair, 1966).

However, as we will discuss in more detail in the following sections, quasars and radio galaxy source counts demonstrated clearly that the populations being studied did evolve strongly with cosmic epoch: the number of quasars per unit comoving volume was clearly larger in the past, so that the information about the geometry of the universe and the cosmological parameters is buried underneath that about the evolution of the AGN themselves.

Progress in characterizing the intrinsic evolution of the QSO population effectively quenched the hope to use black holes as ideal tracers of the structure of the universe, but opened up the study of the evolution of growing supermassive black holes, that we outline below.

\subsection{From Number Counts to Luminosity Functions}

By number counts one typically means the surface density in the sky of a given class of sources as a function of the limiting flux of the observations. In astronomy, this is the simplest observational tool that can be used to study the evolution of a sample of objects (and to test cosmological 


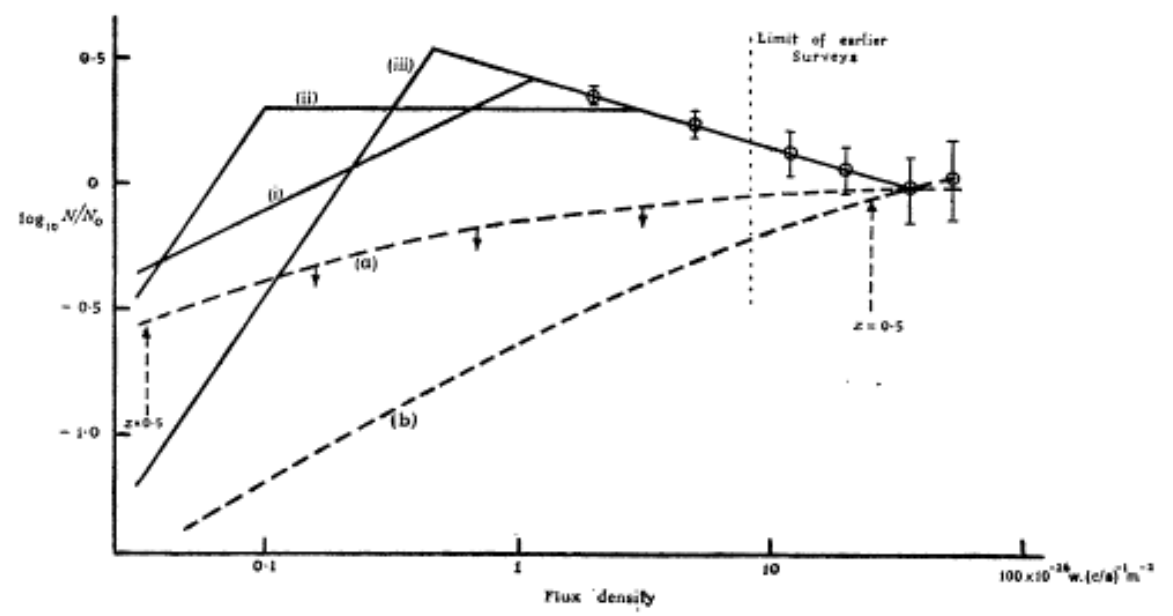

Figure 6: Observed normalized radio number counts from the original 3C catalog at $178 \mathrm{MHz}$ (from Ryle \& Clarke 1961). The observational points (open circles) are extrapolated at low fluxes with three empirical models (i,ii, and iii) made such as not to violate the total lowfrequency radio background available at the time. Dashed lines marked with (a) and (b) denote the counts predicted by the steady state cosmological model assuming two possible luminosity functions, given the observed sources. A clear discrepancy emerged between observations and non-evolving universe models. A more detailed discussion of current constraints on radio sources number counts is given in section 2.1.1 
models).

The space density of sources of different intrinsic luminosities, $L$, is described by the luminosity function (LF), $\phi(L)$, so that $d N=\phi(L) d L$ is the number of sources per unit volume with luminosity in the range $L$ to $L+d L$. Let us, for simplicity, consider the local or nearby (Euclidean) universe uniformly filled with sources with LF $\phi(L)$. If $S$ is the limiting flux that we can detect, sources with luminosity $L$ can be observed out to a distance $r=(L / 4 \pi S)^{1 / 2}$. The number of sources over the solid angle $\Omega$, observable down to the flux $S$ are:

$$
N(>S)=\int \frac{\Omega}{3} r^{3} \phi(L) d L=\frac{\Omega}{3(4 \pi)^{3 / 2}} S^{-3 / 2} \int L^{3 / 2} \phi(L) d L .
$$

Thus, independent of the exact shape of the luminosity function entering in the determination of a normalization constant, the slope of the cumulative number counts of any non-evolving class of sources in a uniform, Euclidean universe should always be equal to $d \log N(>S) / d \log S=$ $-3 / 2$ (if we use magnitudes, $m$, instead of luminosities, then $d \log N(>m) / d m=0.6$ ).

In general, the correct relativistic expression for number counts differs from the Euclidean one because (a) the observed flux density depends upon the spectrum of the source, as the radiation emitted at frequency $\nu_{1}$ is observed at the redshifted frequency $\nu_{0}=\nu_{1} /(1+z)$, and (b) curvature effects modify the volume element per unit redshift, making it smaller with increasing $z$. Overall, for typical source spectra which are not too strongly "inverted" (i.e. with flux density increasing with frequency), the combination of these effects makes it more and more difficult to detect sources at progressively higher redshift and causes number counts to have slopes always shallower than the Euclidean one (see e.g. Longair 2008, chapter 17). As we will see below, strong evolutionary effects (i.e. luminosity functions changing rapidly with time) can counteract such a behavior.

Before proceeding, a brief introduction of common terminology widely adopted in the study of luminosity function evolution is necessary. The simplest general approach describes an evolving luminosity function with the aid of two functions, $f_{1}(z)$ and $f_{\mathrm{d}}(z)$, that take into account the evolution of the luminosity and number density of the sources, respectively:

$$
\phi(L, z)=f_{\mathrm{d}}(z) \phi\left(L / f_{\mathrm{l}}(z), z=0\right) .
$$

In the pure luminosity evolution ( $P L E)$ case $\left(f_{\mathrm{d}}=\right.$ const.), the co-moving number density of sources is constant, but luminosity varies with cosmic epoch; in the pure density evolution (PDE) case $\left(f_{1}=\right.$ const.), but the co-moving density of sources of any luminosity varies.

In the following sections we will discuss the observational state of the art as far as AGN number counts and luminosity functions are concerned, in the radio, X-rays and optical/IR bands. More comprehensive and specialized reviews have, of course, been published. In particular, we refer to the recent work by de Zotti et al. (2010) for a discussion of observations at radio wavelengths, Croom et al. (2009) for optical QSOs and to Brandt \& Hasinger (2005) for X-ray studies.

\subsubsection{The evolution of radio AGN}

Figure 7 shows a compilation of cumulative source number counts from a large number of surveys in different radio bands (data points from Massardi et al. 2010, see references therein). 
On the bottom x-axis, the total radio flux is expressed as $S_{\mathrm{R}} \equiv \nu S_{\nu}$ (where $S_{\nu}$ is the observed radio flux density at any given radio frequency $\nu$ ) in cgs units, while the top axis shows the corresponding radio flux density at $1.4 \mathrm{GHz}$. Overall, the shape of the radio counts is similar in all bands, indicating the relative lack of spectral complexity of radio AGN. This is best seen when normalizing the observed counts to the Euclidean slope, as shown in the top panel.

At bright fluxes, counts rise more steeply than $S^{-3 / 2}$. This was already discovered by the first radio surveys at meter wavelengths (Rvle \& Scheuer, 1955), as we have discussed above, lending strong support for evolutionary cosmological models, as opposed to theories of a steady state universe (see Fig. 6).

At fluxes fainter than about a Jansky $\left(\right.$ or $\approx 10^{-14} \mathrm{ergs} \mathrm{s}^{-1} \mathrm{~cm}^{-2}$ at $1 \mathrm{GHz}$ ) the counts increase less steeply than $S^{-3 / 2}$, being dominated by sources at high redshift, thus probing a substantial volume of the observable universe.

At flux densities above a mJy the population of radio sources is largely composed by AGN. For these sources, the observed radio emission includes the classical extended jet and double lobe radio sources as well as compact radio components more directly associated with the energy generation and collimation near the central engine.

The deepest radio surveys, however, (see e.g. Padovani et al. 2009 and references therein), probing well into the sub-mJy regime, clearly show a further steepening of the counts. The nature of this change is not completely understood yet, but in general it is attributed to the emergence of a new class of radio sources, most likely that of star-forming galaxies and/or radio quiet AGN. Unambiguous solutions of the population constituents at those faint flux levels requires not only identification of the (optical/IR) counterparts of such faint radio sources, but also a robust understanding of the physical mechanisms responsible for the observed emission both at radio and optical/IR wavelengths.

Thus, the complex shape of the observed number counts provides clues about the evolution of radio AGN, as well as on their physical nature, even before undertaking the daunting task of identifying substantial fractions of the observed sources, determining their distances, and translating the observed density of sources in the redshift-luminosity plane into a (evolving) luminosity function. Pioneering work from Longair (1966) already demonstrated that, in order to reproduce the narrowness of the observed 'bump' in the normalized counts around 1 Jy seen in Fig. 7, only the most luminous sources could evolve strongly (in density and/or luminosity) with redshift. This was probably the first direct hint of the intimate nature of the differential evolution AGN undergo over cosmological times.

Indeed, many early investigations of high redshift radio luminosity functions (see, e.g., Danese et al. 1987) demonstrated that neither PLE nor PDE models could explain the observed evolution of radio sources, with more powerful sources (often of FRII morphology) displaying a far more dramatic rise in their number densities with increasing redshift (see also Willott et al. 2001).

Trying to assess the nature of radio AGN evolution across larger redshift ranges requires a careful evaluation of radio spectral properties of AGN. Steeper synchrotron spectra are produced in the extended lobes of radio jets, while flat spectra are usually associated with compact cores. For objects at distances such that no radio morphological information is available, the

\footnotetext{
${ }^{3}$ A Jansky (named after Karl Jansky, who first discovered the existence of radio waves from space) is a flux measure, corresponding to $10^{-23}$ ergs $\mathrm{cm}^{-2} \mathrm{~Hz}^{-1}$
} 


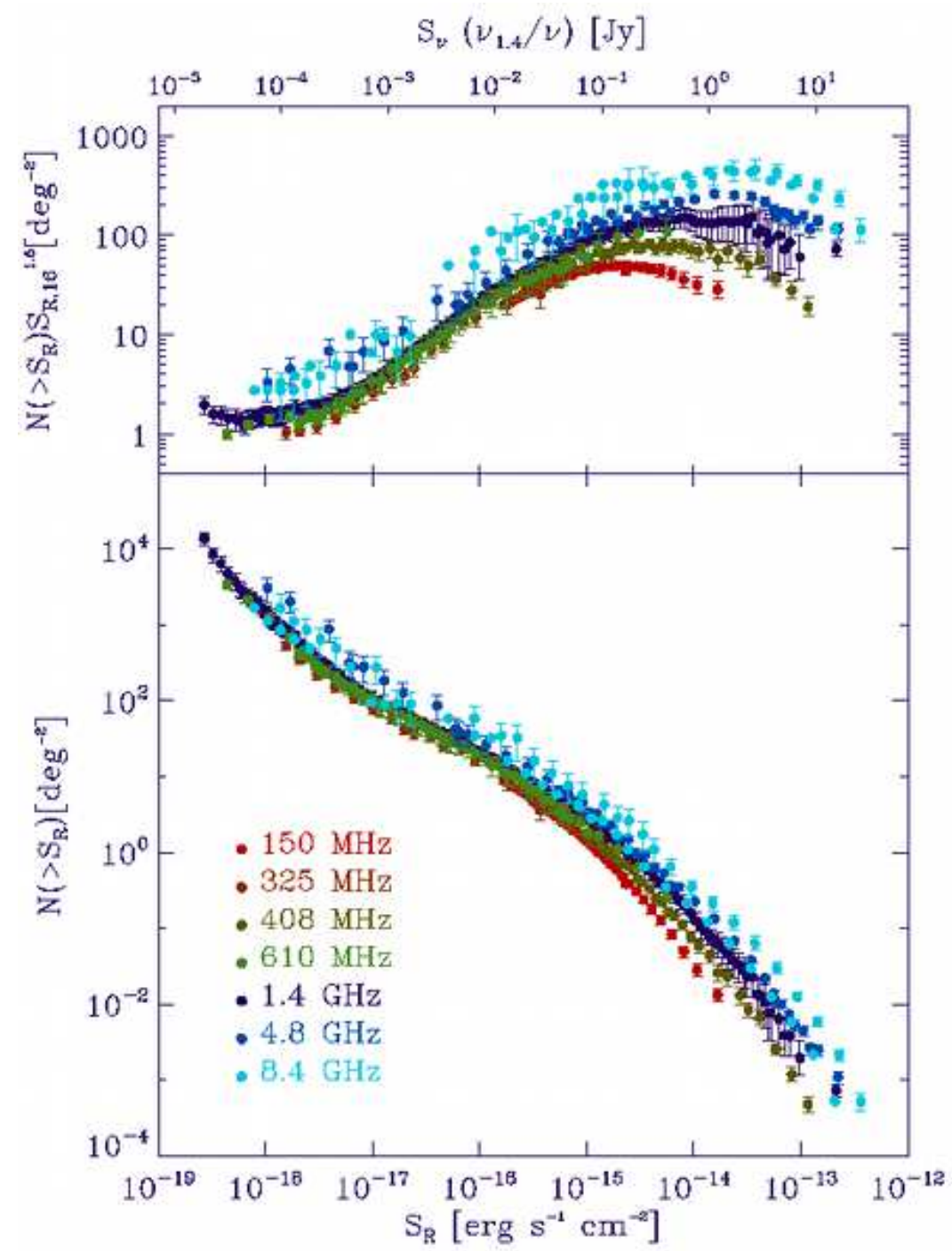

Figure 7: A compilation of cumulative radio source counts (number of objects brighter than a given flux per square degree) in various bands. The observational data are taken from (Massardi et al. 2010 see also references therein). On the bottom horizontal axis the total radio flux $S_{\mathrm{R}}=\nu S_{\nu}$ in CGS units is shown, while the top horizontal axis shows the corresponding flux density in Jansky, where $\nu_{1.4}$ is the frequency of $1.4 \mathrm{GHz}$. The bottom panel shows the observed counts, while the top panel shows the counts after the Euclidean slope has been factored out. 

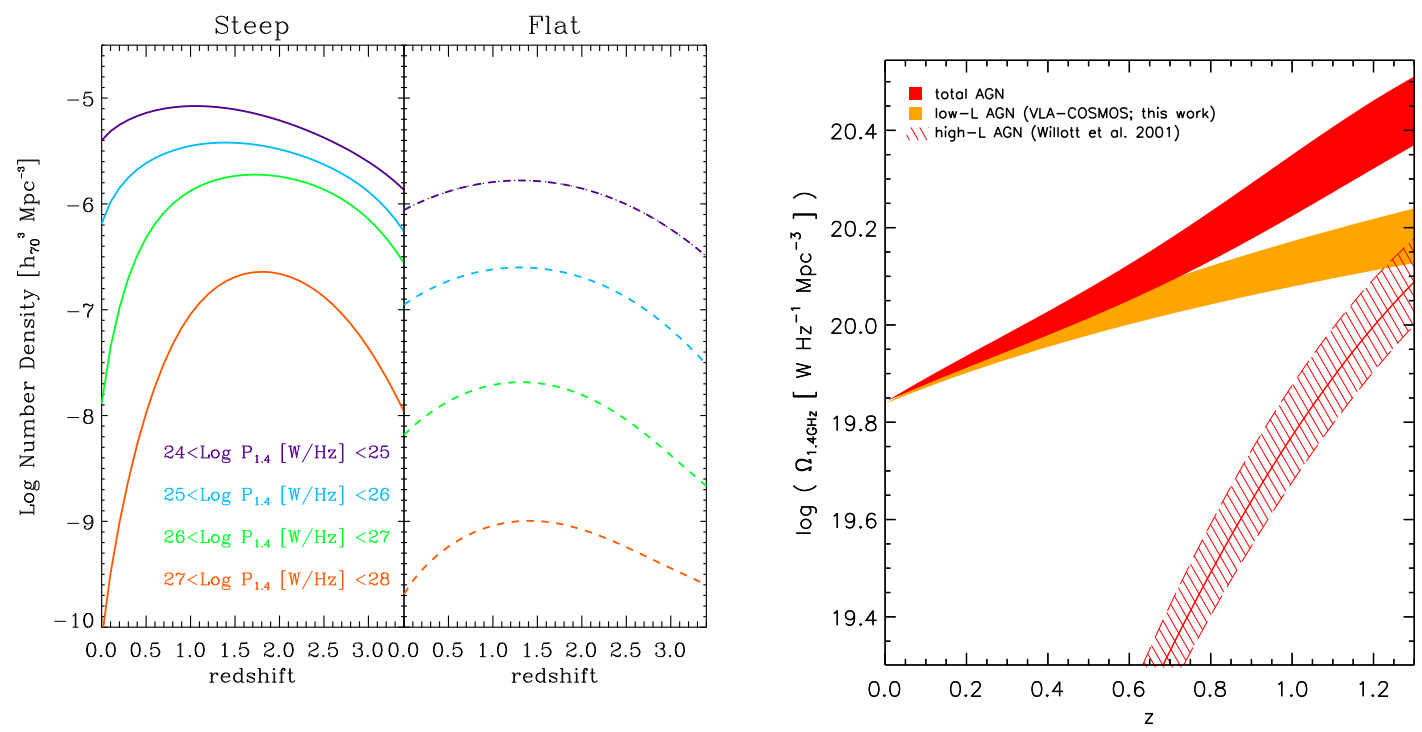

Figure 8: The radio view of AGN downsizing. Left Best fit number density evolution of radio sources of different power, taken from the models of Massardi et al. (2010), for steep and flat spectrum sources in the left and right panels, respectively. Right: Evolution of the comoving 20 $\mathrm{cm}$ integrated luminosity density for VLA-COSMOS AGN (orange curve) galaxies for $z<1.3$. Also shown is the evolution of the high-luminosity radio AGN, adopted from (Willott et al. 2001, hatched region; the thick and dashed lines correspond to the mean, maximum and minimum results, respectively). The evolution for the total AGN population, obtained by co-adding the VLA-COSMOS and high luminosity AGN energy densities, is shown as the red-shaded curve (adopted from Smolčić et al. 2009). 
combination of observing frequency, K-corrections, intrinsic source variability and orientation of the jet with respect to the line of sight may all contribute to severe biases in the determination of the co-moving number densities of sources, especially at high redshift (Wall et al., 2005).

In a very extensive and equally influential work Dunlop \& Peacock (1990) studied the evolution of the luminosity functions of steep and flat spectrum sources separately. They showed that the overall redshift evolution of the two classes of sources were similar, with steep spectrum sources outnumbering flat ones by almost a factor of ten. Uncertainties remained regarding the possibility of a high-redshift decline of radio AGN number densities. The issue is still under discussion, with the most clear evidence for such a decline observed for flat-spectrum radio QSO at $z>3$ (Wall et al., 2005), consistent with the most recent findings of optical and X-ray surveys (see also $\S 5$ below).

Under the simplifying assumption that the overall radio AGN population can be sub-divided into steep and flat spectrum sources, characterized by a power-law synchrotron spectrum $S_{\nu} \propto$ $\nu^{-\alpha}$, with slope $\alpha_{\text {flat }}=0.1$ and $\alpha_{\text {steep }}=0.8$, respectively, a redshift dependent luminosity function can be derived for the two populations separately, by fitting simple models to a very large and comprehensive set of data on multi-frequency source counts and redshift distributions obtained by radio surveys at $\nu<5 \mathrm{GHz}$ (Massardi et al., 2010). The comoving number densities in bins of increasing radio power (at $1.4 \mathrm{GHz}$ ) from the resulting best fit luminosity function models are shown in the left panel of Figure 8.

Radio AGN, both with steep and flat spectrum, show the distinctive feature of a differential density evolution, with the most powerful objects evolving more strongly towards higher redshift, a phenomenological trend that, in the current cosmologist jargon, is called "downsizing".

Recent radio observational campaigns of large multi-wavelength sky surveys have also corroborated this view, by providing a much more detailed picture of low luminosity radio AGN. For example, the work of Smolčić et al. (2009) on the COSMOS field showed that radio galaxies with $L_{1.4 \mathrm{GHz}}<$ few $\times 10^{25} \mathrm{WHz}^{-1}$ evolve up to $z \simeq 1$, but much more mildly than their more luminous counterparts, as shown in the right panel Figure 8 .

\subsubsection{X-ray surveys and the resolution of the X-ray background}

As already mentioned, active galactic nuclei are powerful X-ray emitters. The discovery of the intense cosmic X-ray background radiation (CXRB; Giacconi et al., 1962) in the early 1960's opened up a privileged window for the study of the energetic phenomena associated with accretion onto black holes.

Due to the relative weakness of X-ray emission from stars and stellar remnants (magnetically active stars, cataclysmic variables and, more importantly, X-ray binaries are the main stellar Xray sources), the X-ray sky is almost completely dominated by the evolving SMBH population, at least down to the faintest fluxes probed by current X-ray focusing telescopes. The goal of reaching a complete census of evolving AGN, and thus of the accretion power released by SMBH in the history of the universe has therefore been intertwined with that of fully resolving the CXRB into individual sources. Accurate determinations of the CXRB intensity and spectral shape, coupled with the resolution of this radiation into individual sources, allow very sensitive tests of how the AGN luminosity and obscuration evolve with redshift.

New generations of synthesis models of the CXRB (Gilli et al., 2007; Treister et al., 2009) have quickly followed the publication of increasingly larger and deeper surveys (for the current 


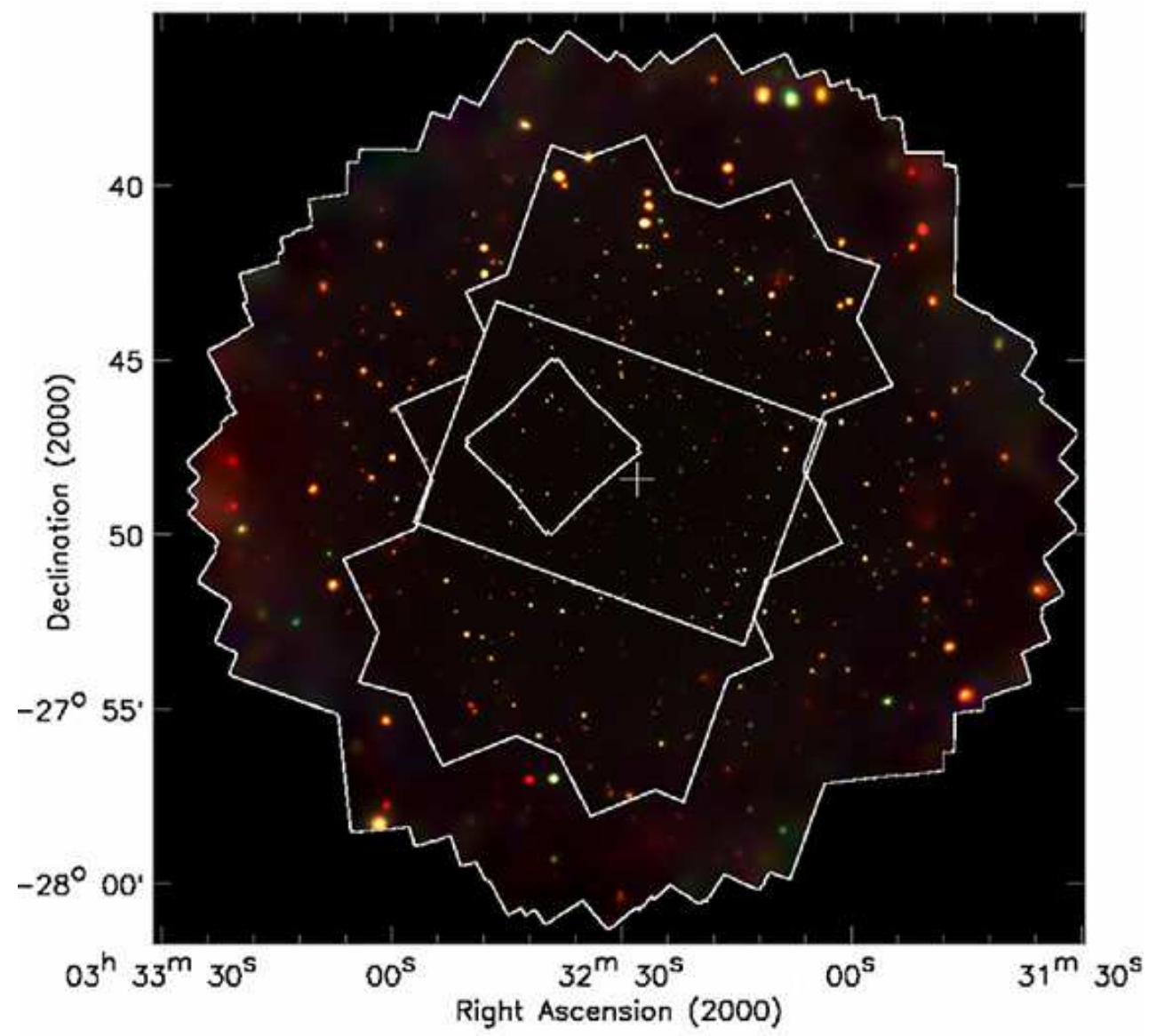

Figure 9: A smoothed, false color image of the deepest X-ray exposure to date, the $4 \times 10^{6}$ second Chandra observation of the Chandra Deep Field South (CDFS). These observations resolve almost the entire CXRB radiation below $\sim 5 \mathrm{keV}$ into individual sources, the vast majority of which are accreting supermassive black holes. From Xue et al. (2011). 
deepest view of the X-ray sky, see Figure 91). Figure 10 shows a recent compilation of the CXRB measurements together with one incarnation of a synthesis model (Treister et al., 2009) of AGN evolution that explains those data. The hard slope of the background spectrum (well described by a power-law with photon index $\Gamma_{\mathrm{CXRB}} \simeq 1.4$ at $E<10 \mathrm{keV}$ ) and the prominent peak at about $30 \mathrm{keV}$ are accounted for by assuming that the majority of active galactic nuclei are in fact obscured.

These new models have progressively reduced the uncertainties in the absorbing column density distribution. When combined with the observed X-ray luminosity functions, they provide an almost complete census of the Compton thin AGN (i.e., those obscured by columns $N_{\mathrm{H}}<$ $\sigma_{\mathrm{T}}^{-1} \simeq 1.5 \times 10^{24} \mathrm{~cm}^{-2}$, where $\sigma_{\mathrm{T}}$ is the Thomson cross section). This class of objects dominates the counts in the lower energy X-ray energy band, where almost the entire CXRB radiation has been resolved into individual sources (Worsley et al., 2005). It should be noted, however, that at the peak energy of the observed CXRB radiation only a small fraction (less than 5\%) of the emission has so far been resolved into individual objects.

CXRB synthesis models, like the one shown in Figure 10 ascribe a substantial fraction of this unresolved emission to heavily obscured (Compton Thick) AGN. However, because of their faintness even at hard X-ray energies, their redshift and luminosity distribution is essentially unknown, and even their absolute contribution to the overall CXRB sensitively depends on the quite uncertain normalization of the unresolved emission at hard X-ray energies. The quest for the physical characterization of this "missing" AGN population, most likely dominated by Compton thick AGN, represents one of the last current frontiers of the study of AGN evolution at X-ray wavelengths.

Putting together the observational data from a large suite of complementary surveys, Figure 11 shows a compilation of X-ray number counts, for both soft (0.5-2 keV) and hard (2-10 $\mathrm{keV}$ ) selected samples.

Given the steep frequency dependence of photo-electric absorption cross sections, the harder the energy band probed the less affected by obscuration the objects under study are. Current technologies provide the best compromise between telescope effective area and energy range in the $2-10 \mathrm{keV}$ band. Indeed, the density of AGN detected in this band is higher than that of $0.5-2 \mathrm{keV}$ selected ones, by at least a factor of 3 .

As for the radio $\log N-\log S$ of Fig. 7, the counts become shallower than the Euclidean slope at intermediate fluxes (about $10^{-14} \mathrm{ergs} \mathrm{s}^{-1} \mathrm{~cm}^{-2}$ ), where the largest relative fraction of the CXRB is produced. Tentative evidence of a steepening at the lowest fluxes might indicate the emergence of a different, non-AGN, population of star-forming galaxies whose X-ray emission is primarily due to stars and stellar remnants.

The deepest surveys so far carried out in the soft X-ray energy range $(0.5-2 \mathrm{keV})$, supplemented by the painstaking work of optical identification and redshift determination of the detected sources have provided the most accurate description of the overall evolution of the AGN luminosity function. Neither PLE nor PDE provide a satisfactory description of the X-ray LF evolution, with a good fit to the data achieved with a "Luminosity Dependent Density Evolution" (LDDE) model, or variations thereof. In their influential work, Hasinger et al. (2005) unambiguously demonstrated that in the observed soft X-ray energy band more luminous AGN peaked at higher redshift than lower luminosity ones (see Fig. 12).

A good enough sampling of the luminosity redshift plane necessary for accurate LF studies 


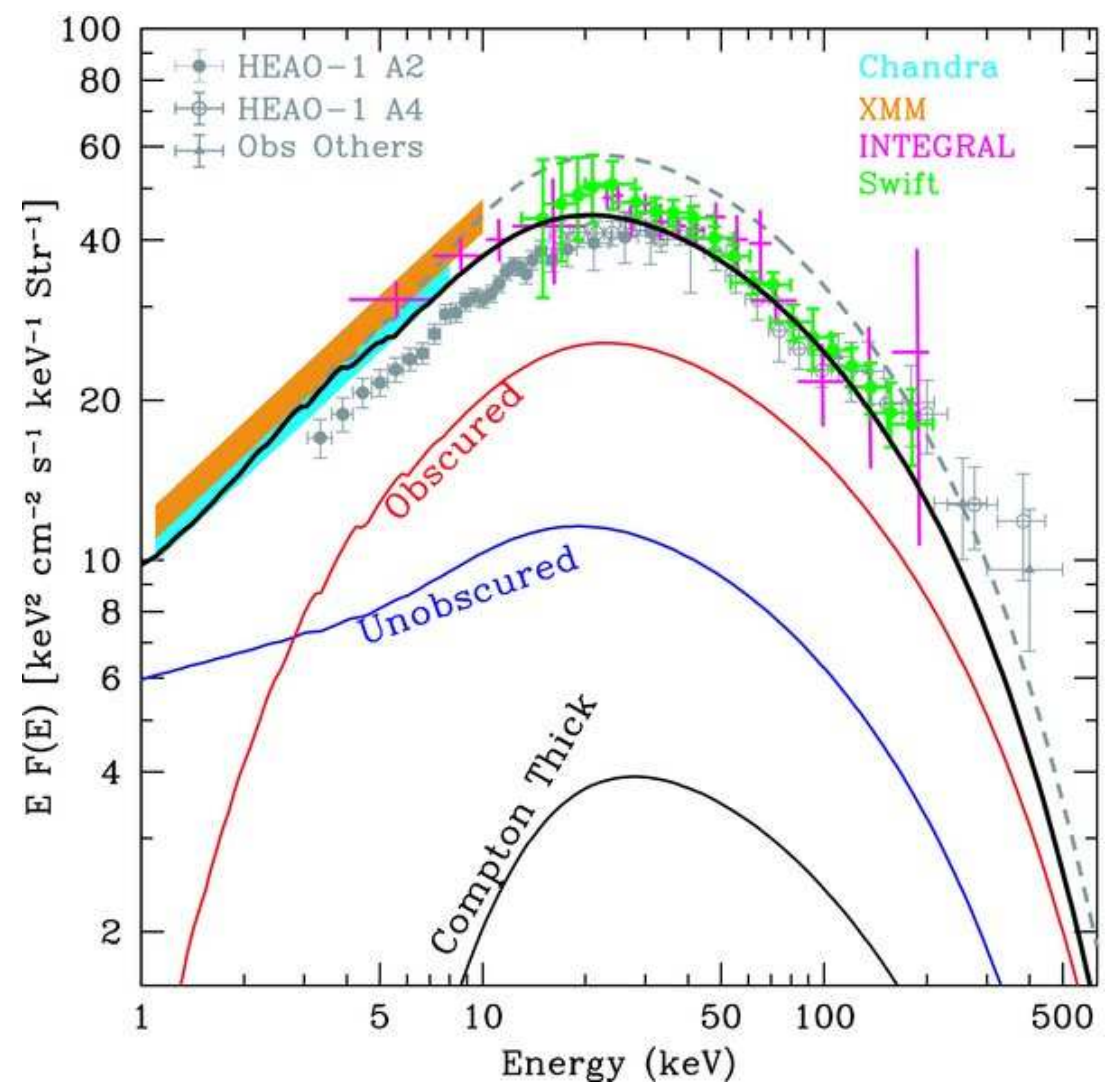

Figure 10: Observed spectrum of the extragalactic CXRB from HEAO 1, Chandra, XMMNewton, INTEGRAL, and Swift data. The solid (red, blue, and black) thin lines show the contribution to this model from unobscured, obscured Compton-thin, and Compton Thick AGNs, respectively. The thick black solid and dashed grey lines are two different CXRB spectral models proposed in the literature depending on the assumed normalization of the HEAO 1; the main difference is the number of Compton Thick AGNs, which is reduced by a factor of 4 if the black solid line is assumed instead of the dashed one. Adopted from Treister et al. (2009), where a complete list of references of the data points shown is presented. 


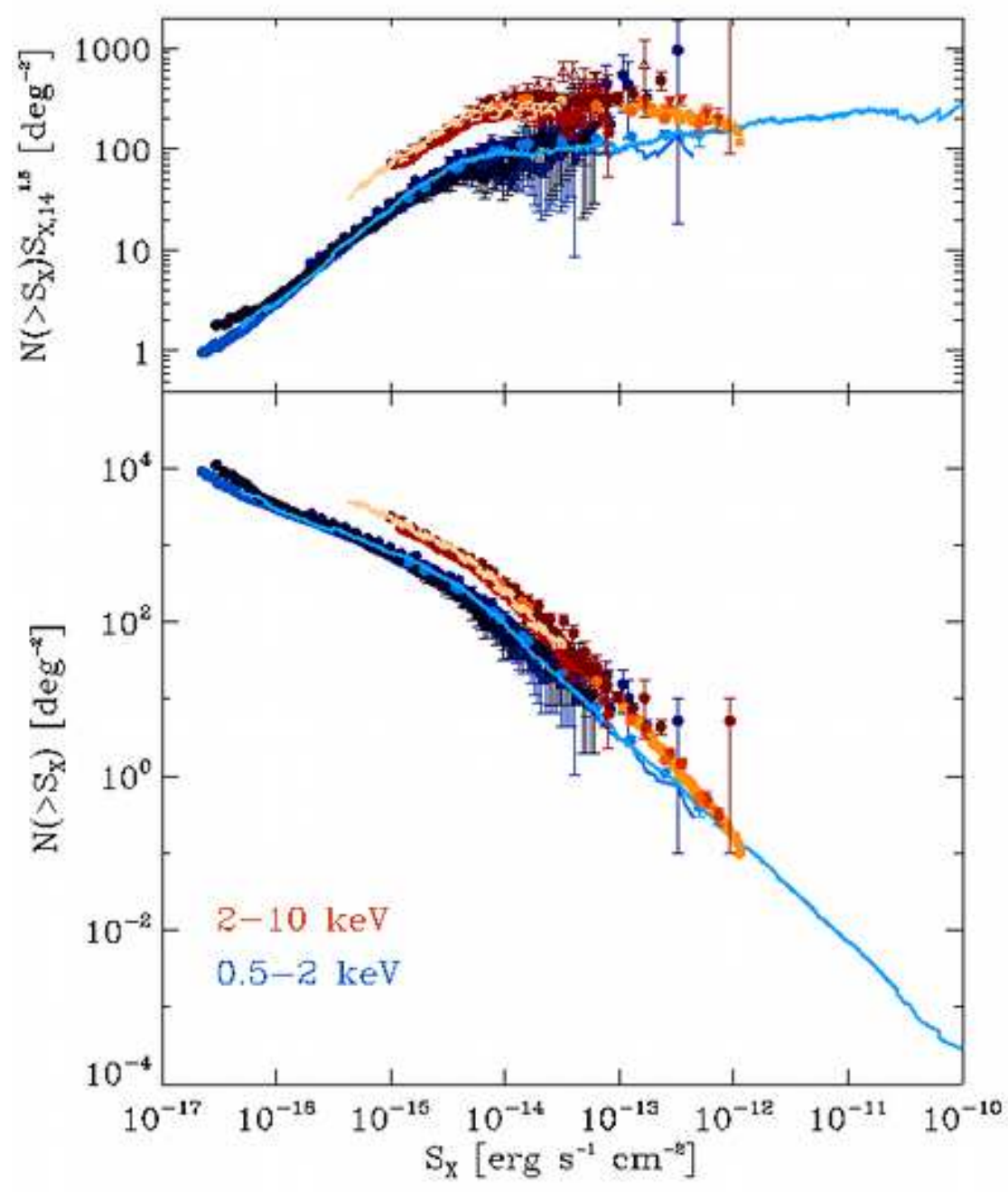

Figure 11: A compilation of cumulative X-ray source counts (number of objects brighter than a given flux per square degree) in the soft $(0.5-2 \mathrm{keV}$, cyan-blue colors) and hard (2-10 keV, red-orange colors) bands. The observational data are taken from Cappelluti et al. (2009) (see references therein) and Mateos et al. (2008). The bottom panel shows the observed counts, while the top panel shows the counts after the Euclidean slope has been factored out. 

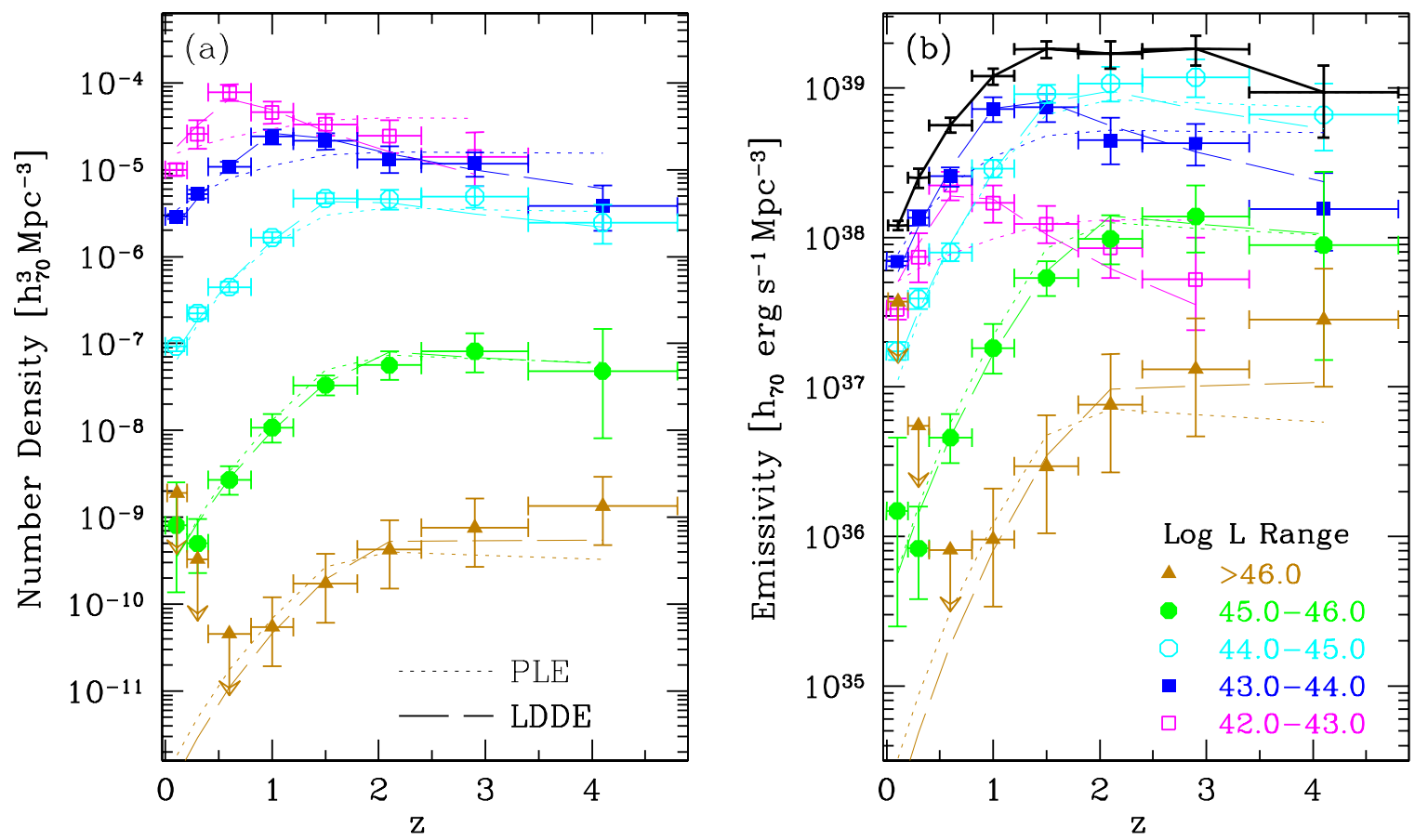

Figure 12: The soft X-ray view of AGN downsizing: (a) The space density of AGNs as a function of redshift in different luminosity classes and the sum over all luminosities with $\log L_{\mathrm{x}} \gtrsim 42$. Densities from the PLE and LDDE models are over-plotted with solid lines. (b) The same as (a), except that the soft X-ray emissivities are plotted instead of number densities. The uppermost curve (black) shows the sum of emissivities in all luminosity classes in the plot. From Hasinger et al. (2005). 
requires more extensive observational efforts in the hard X-ray band, as obscured AGN are more difficult to identify (and to obtain redshifts for) in the optical band. Nonetheless, the general "downsizing" trend illustrated by the soft-X-ray selected AGN of Fig. 12 has so far been confirmed by almost all recent studies of (2-10 keV) X-ray selected AGN (see e.g. Ueda et al., 2003).

\subsubsection{Optical and Infrared studies of QSOs}

Bright AGN emit a large fraction of their bolometric luminosity in the optical/UV part of the spectrum (see Chapter "Active Galactic Nuclei" by E. Perlman in this volume). For Eddington ratios $\left(\lambda \equiv L_{\mathrm{bol}} / L_{\mathrm{Edd}}\right.$, where $L_{\mathrm{Edd}}=4 \pi G M_{\mathrm{BH}} m_{\mathrm{p}} c / \sigma_{\mathrm{T}} \simeq 1.3 \times 10^{38}\left(M_{\mathrm{BH}} / M_{\odot}\right) \mathrm{ergs} \mathrm{s}^{-1}$ is the Eddington luminosity) larger than a few per-cent, the AGN light out-shines the emission from the host galaxy, resulting in point-like emission with peculiar blue colors.

Finding efficient ways to select QSO in large optical surveys, trying to minimize contamination from stars, white dwarfs and brown dwarfs has been a primary goal of optical astronomers since the realization that QSO were extragalactic objects often lying at cosmological distances (Schmidt \& Green, 1983; Richards et al., 2006).

Optical surveys remain an extremely powerful tool to uncover the evolution of unobscured QSOs up to the highest redshift $(z \sim 6)$. In terms of sheer numbers, the known population of SMBH is dominated by such optically selected AGN (e.g. more that $10^{5}$ QSOs have been identified in the Sloan Digital Sky Survey), essentially due to the yet unsurpassed capability of ground-based optical telescopes to perform wide-field, deep surveys of the extra-galactic sky.

Figure 13 shows a compilation of (g-band, $\approx 4700 \AA$ ) quasar number counts from the largest recent optical surveys (2SLAQ, 2QZ, and SDSS), for objects in the redshift range $0.3<z<2.2$. The overall shape is similar to that of the radio AGN counts, with a steep increase at bright fluxes, followed by a turnover at around $g \simeq 19$. A comparison with Figures 7 and 11 reveals, however, that such large area QSO surveys reach depths corresponding to a number density of sources in the sky more than one order of magnitudes smaller that those probed by the deepest $\mathrm{X}$-ray and radio surveys.

Thus, the dominant AGN population eludes systematic detection in optical surveys. In general terms, the difficulty of optical QSO surveys to probe deep into the AGN population is due to two major effects: the first one is the already mentioned issue of nuclear obscuration, dramatically affecting the UV/optical appearance of AGN; the second is galaxy dilution of the AGN light (and of the broad emission line signature often used to select quasars). More specifically, let us consider an AGN with B-band luminosity given by $L_{\mathrm{AGN}, \mathrm{B}}=\lambda L_{\mathrm{Edd}} f_{\mathrm{B}}$, with bolometric correction $f_{\mathrm{B}} \approx 0.1$ (Richards et al., 2006). Assuming a bulge-to-black hole mass ratio of 0.001 and a bulge-to-total galactic stellar mass ratio of $(B / T)$, the contrast between nuclear AGN continuum and host galaxy blue light is given by:

$$
\frac{L_{\mathrm{AGN}, \mathrm{B}}}{L_{\mathrm{host}, \mathrm{B}}}=\frac{\lambda}{0.1} \frac{\left(M_{*} / L_{\mathrm{B}}\right)_{\mathrm{host}}}{3\left(M_{\odot} / L_{\odot}\right)}(B / T)
$$

Thus, for typical mass-to-light ratios, the AGN will become increasingly diluted by the host stellar light at Eddington ratios $\lambda$ smaller than a few per cent.

High-spatial resolution observations of the numerically dominant population of Low-Luminosity AGN (LLAGN; see the comprehensive review of $\mathrm{Ho}$ 2008, and references therein) have so far 


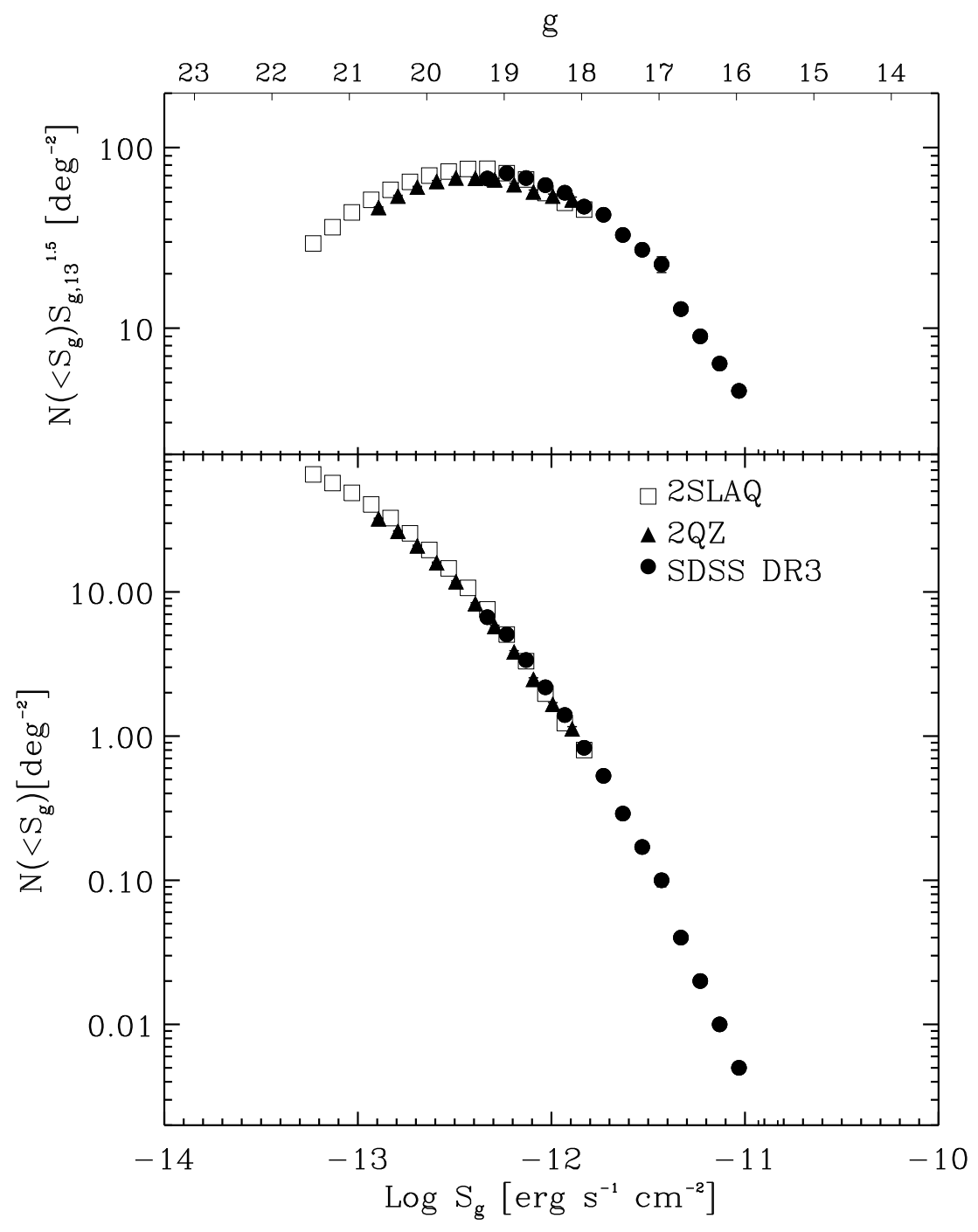

Figure 13: Compilation of (g-band, $\approx 4700 \AA$ ) quasar number counts from the largest recent optical surveys at $0.3<z<2.3$ (2SLAQ, 2QZ and SDSS, courtesy of G. Richards and S. Croom). g-band magnitudes have been converted into CGS flux units $\left(S_{g}\right)$ with $\log S_{g}=-0.4(g-20)-$ 12.64 . 
only been possible in the very local universe. At higher redshift, the deepest multi-wavelength AGN/galaxy surveys to date are starting to probe AGN luminosities such that the contribution of the host galaxy to the overall SED cannot be neglected. This compromises the efficiency and "cleanness" of AGN selection at optical/IR wavelength, but opens up the possibility of studying the connection between nuclear black hole activity and host galaxy properties. We will come back more extensively in section 3.2 to the issue of the overall decomposition of the AGN-galaxy spectral energy distribution in large multiwavelength surveys.

As for the general evolution of the optically selected QSO luminosity function, it has been known for a long time that luminous QSOs were much more common at high redshift $(z \sim 2)$. Nevertheless, it is only with the aid of the aforementioned large and deep surveys covering a wide enough area of the distance-luminosity plane that it was possible to put sensible constraints on the character of the observed evolution. The most recent attempts (Croom et al., 2009) have shown unambiguously that optically selected AGN do not evolve according to a simple PLE, but instead more luminous objects peaked in their number densities at redshifts higher than lower luminosity objects, as shown in the left panel of Figure 14.

We close this section with a brief discussion of the current status of IR AGN LF studies.

According to the AGN unification paradigm, obscuration comes from optically thick dust blocking the central engine along some lines of sight. The temperature in this structure, which can range up to $1000 \mathrm{~K}$ (the typical dust sublimation temperature), and the roughly isotropic emission toward longer wavelengths should make both obscured and unobscured AGNs very bright in the mid- to far-infrared bands. This spectral shift of absorbed light to the IR has allowed sensitive mid-infrared observatories (IRAS, ISO, Spitzer) to deliver large numbers of AGN (see, e.g. Treister et al., 2006).

Traditionally, the problem with IR studies of AGN evolution, however, lies neither in the efficiency with which growing supermassive black holes can be found, nor with the completeness of the AGN selection, which is clearly high and (almost) independent of nuclear obscuration, but rather in the level of contamination. IR counts are, in fact, dominated by star forming galaxies at all fluxes. This, and the lack of clear spectral signatures in the nuclear, AGN-powered emission in this band, implies that secure identification of AGN in any IR-selected catalog often necessitates additional information from other wavelengths, usually radio, X-rays, or optical spectroscopy.

Indeed, unlike the case of the CXRB, AGN contribute only a small fraction (up to 2-10\%) of the cosmic IR background radiation (Treister et al., 2006), and similar fractions are estimated for the contribution of AGN at the "knee" of the total IR luminosity function at all redshifts.

Nonetheless, tremendous progress has been achieved in recent years, thanks to more refined mid-IR color-color selection criteria (Stern et al., 2005) which are little affected by contamination, and provide reliable AGN samples, albeit with some well understood completeness biases against AGNs that are faint with respect to their hosts, and z 4.5 Type 1 AGNs.

Thus, deep surveys with extensive multi-wavelength coverage can also be used to track the evolution of active galaxies in the mid-infrared (see, e.g. Assef et al., 2011). Strengthening similar conclusions discussed above from other wavelengths, IR-selected AGN do not appear to evolve following either the PLE or PDE parametrizations, but require significant differences in the evolution of bright and faint sources, with the number density of the former declining more steeply with decreasing redshift than that of the latter (see the right panel in Figure 14). 

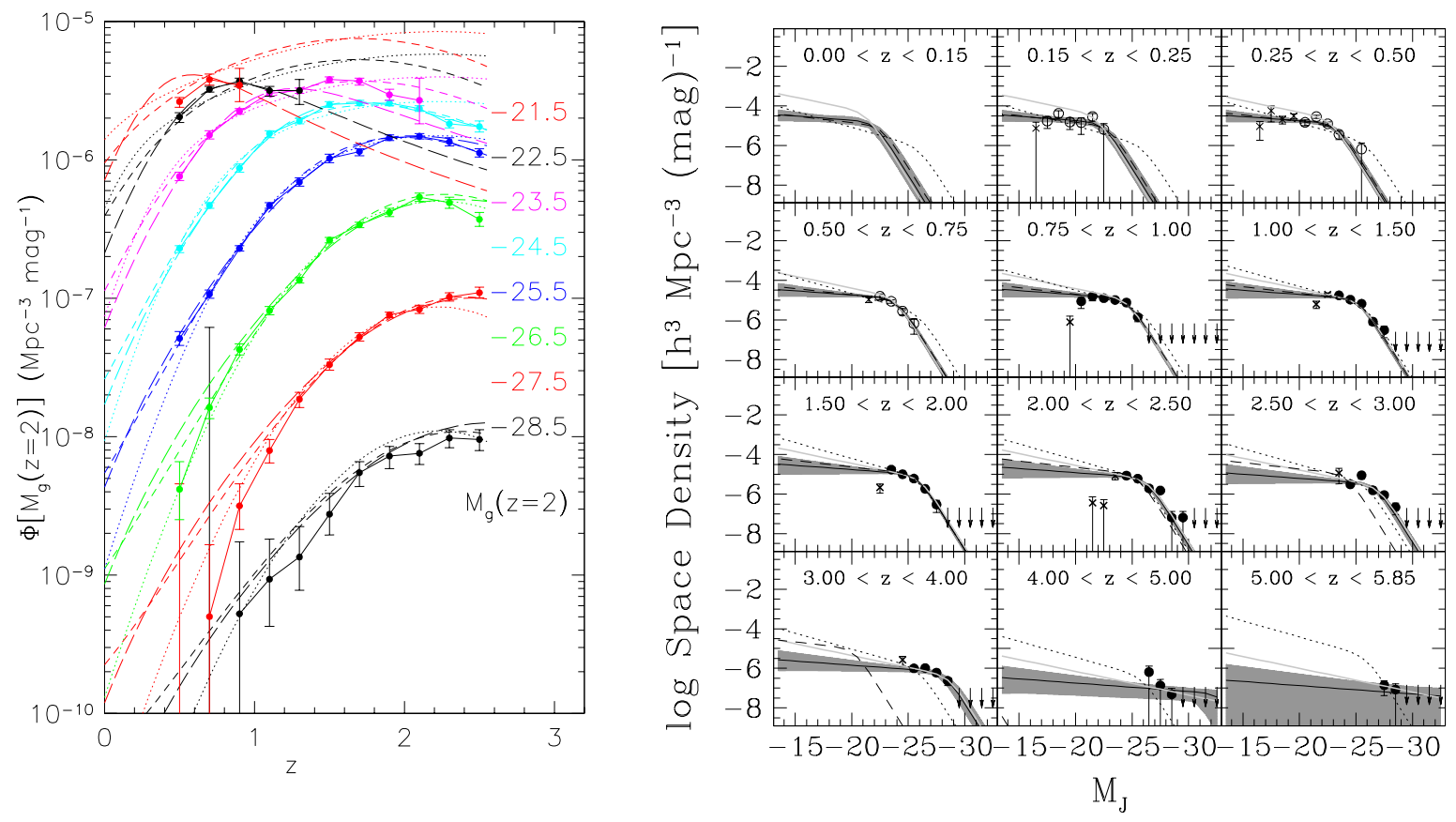

Figure 14: Left: The combined 2SLAQ and SDSS optical QSO luminosity function plotted as a function of redshift for different absolute $g$ band magnitude intervals (the brightest at the bottom of the plot and the faintest at the top). The measured LF is compared to the best fit PLE model (dotted lines), smooth LDDE model (long dashed lines) and LADE (Luminosity And Density Evolution) model (short dashed lines). Adopted from (Croom et al., 2009); Right: J-band luminosity function of mid-IR-selected AGN for several redshift bins. The crosses show points that were not used in the fits. The best-fit LADE, PLE, and pure PDE models are shown by the solid, dashed, and dotted line, respectively, although only the LADE model is an acceptable fit to the data. The shaded area shows the $2 \sigma$ confidence region for the LADE fit. For reference, the solid light gray line shows the best-fit LADE model to a sample from a combined IR/X-ray selection. From Assef et al. (2011). 


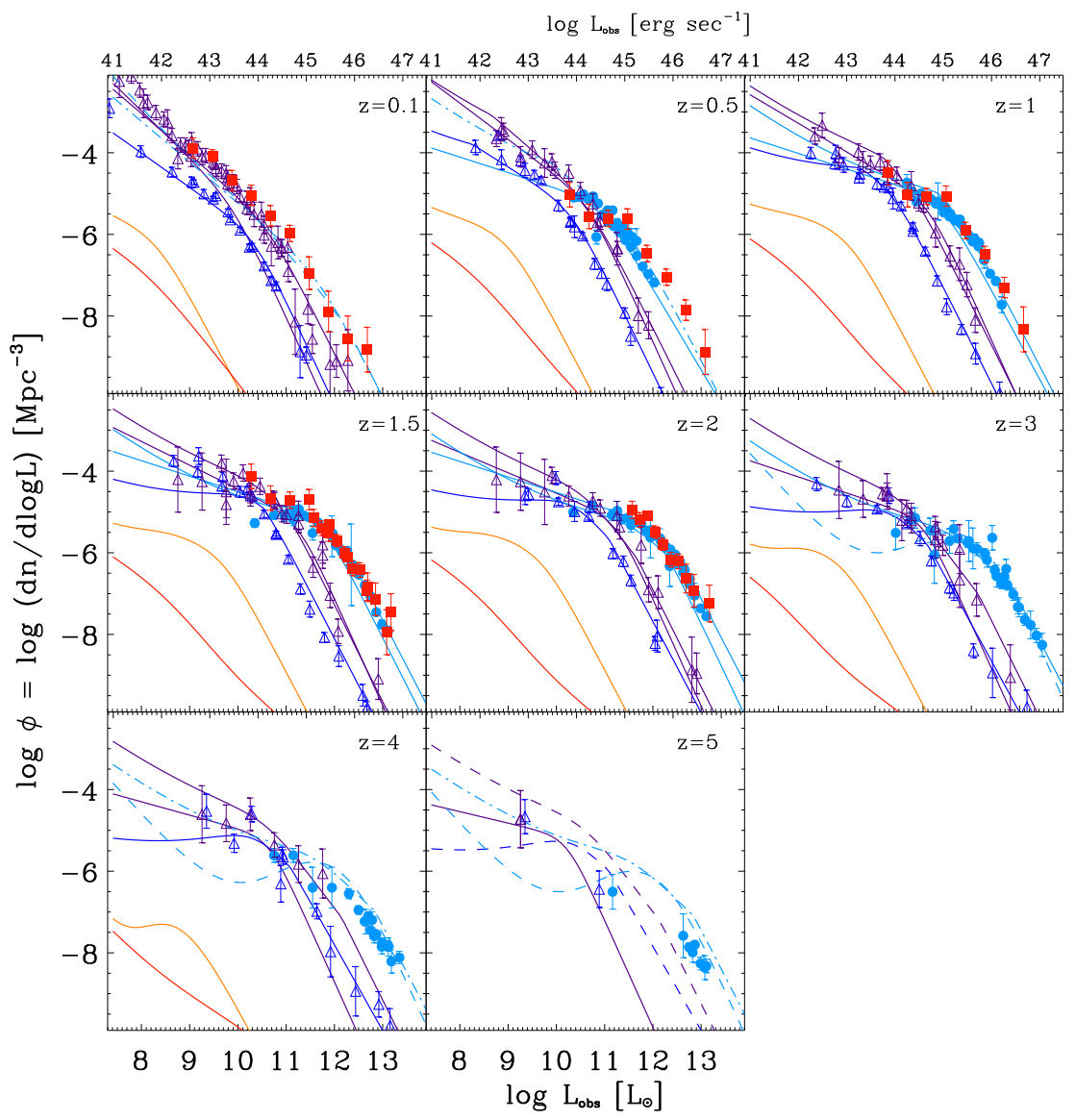

Figure 15: A compilation of luminosity functions observed in various energy bands. The logarithm of the number of AGN per unit comoving volume and unit logarithm of luminosity is plotted as a function of the observed luminosity (in solar units). Observational points for IR ( $15 \mu \mathrm{m}$; filled red squares), B-band (filled blue circles), soft- (0.5-2 keV; empty blue triangles) and hard-X-rays (2-10 keV; empty purple triangles) are shown alongside published analytic fits for each band (solid lines in corresponding colors). The best fit radio luminosity functions of steep and flat spectrum sources from Massardi et al. (2010) are also shown for comparison with orange and red thick lines, respectively. The observed mismatch among the various luminosity functions in Fig. 15 is due to a combination of different bolometric corrections and incompleteness due to obscuration. Courtesy of P. Hopkins 

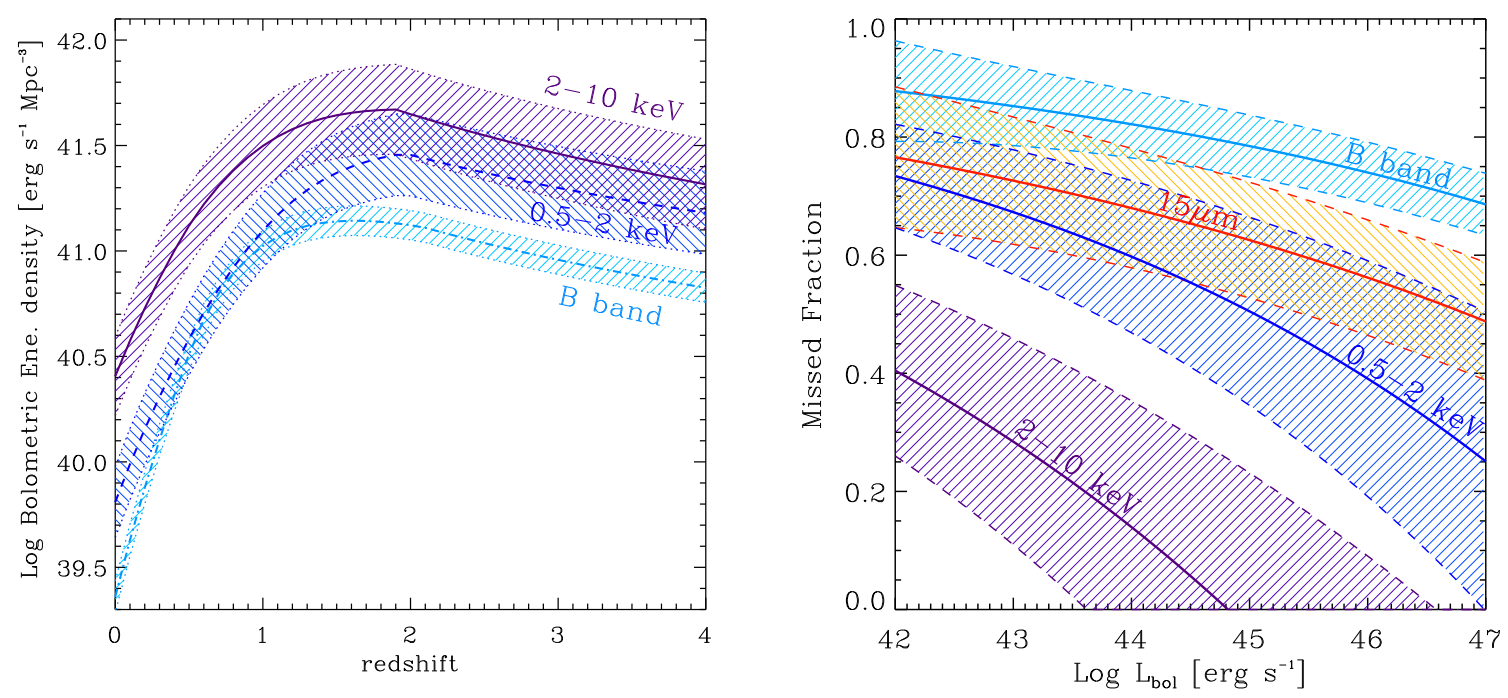

Figure 16: Left: The redshift evolution of the bolometric energy density for AGN selected in different bands. Bolometric corrections from Hopkins et al. (2007) have been used, and the shaded areas represent the uncertainty coming from the bolometric corrections only. Right: The fraction of AGN missed by observations in any specific band as a function of the intrinsic bolometric luminosity of the AGN. Red, light blue, dark blue and purple shaded areas correspond to rest-frame mid-IR $(15 \mu \mathrm{m})$, UV (B-band), soft X-rays $(0.5-2 \mathrm{keV})$ and hard X-rays (2-10 $\mathrm{keV}$ ), respectively. The uncertainty on the missed fractions depend on the uncertainties of the bolometric corrections and on the shape of the observed luminosity functions only.

\subsubsection{Bolometric Luminosity functions}

We have seen in the previous sections how a qualitatively consistent picture of the main features of AGN evolution is emerging from the largest surveys of the sky in various energy bands. Strong (positive) redshift evolution of the overall number density, as well as marked differential evolution (with more luminous sources being more dominant at higher redshift) characterize the evolution of AGN.

Fundamental constraints on the physical evolution of the accretion-powered emission over cosmological times, like the ones we will discuss later in 93 , require, ideally, a good knowledge of the bolometric luminosity function of AGN. This, in turn, demands a detailed assessment of selection biases and a robust estimation of the AGN Spectral Energy Distribution (SED).

A thorough and detailed understanding of the AGN SED as a function of luminosity (and, possibly, of redshift, but see section 1.1 above) could in principle allow us to compare and crosscorrelate the information on the AGN evolution gathered in different bands. As for the accuracy of our knowledge of the bolometric correction, we refer the reader to the studies of Marconi et al. (2004); Richards et al. (2006); Hopkins et al. (2007). All of them consistently demonstrate that a luminosity dependent bolometric correction is required in order to match type I (unabsorbed) AGN luminosity functions obtained by selecting objects in different bands.

Summarizing the discussion of the previous sections, Figure [15] shows a compilation of lu- 
minosity functions observed at various wavelengths. The observed mismatch among the various LF observed at all redshift is due to a combination of different bolometric corrections and incompleteness due to obscuration. In fact, adopting a general form of luminosity-dependent bolometric correction, and with a relatively simple parametrization of the effect of the obscuration bias on the observed LF, Hopkins et al. (2007) were able to project the different observed luminosity functions in various bands into a single bolometric one, $\phi\left(L_{\mathrm{bol}}\right)$ (Figure 17). As a corollary from such an exercise, we can then provide a simple figure of merit for AGN selection in various bands by measuring the bolometric energy density associated with AGN selected in that particular band as a function of redshift. We show this in the left panel of Figure 16 for four specific bands (hard X-rays, soft X-rays, UV, and mid-IR). From this, it is obvious that the reduced incidence of absorption in the $2-10 \mathrm{keV}$ band makes the hard X-ray surveys recover a higher fraction of the accretion power generated in the universe than any other method.

While optical QSO surveys miss more than three quarters of all AGN of any given $L_{\mathrm{bol}}$, hard X-ray selection only fails to account for about one third (up to 50\%) of all AGN, the most heavily obscured (Compton Thick) ones, as shown in the right panel of Figure 16. A common feature apparent from such a figure is that the effects of obscuration appear to be more severe at lower intrinsic luminosities, an observational fact that has been discussed previously in the context of X-ray surveys of AGN (see section 1.1 above). It is important to note that the high missed fraction for mid-IR selected AGN is a direct consequence of the need for (usually optical) AGN identification of the IR sources, so that optically obscured active nuclei are by and large missing in the IR AGN luminosity functions considered here.

Figure 18 shows the evolution of the parameters of the analytic fit to the bolometric LF data. They encompass our global knowledge of the evolution of accretion power onto nuclear black holes throughout the history of the universe. The three bottom panels reveal the overall increase in AGN activity with redshift, up to $z \approx 2$, and the mirroring high-redshift decline. At the center, the total integrated luminosity density evolution mark the epochs of rapid build-up of the SMBH mass density. On the lower left, the evolution in the break luminosity $L_{\mathrm{bol}, *}$ indicates that the "typical" accreting black holes was significantly more luminous at $z \approx 2$ than now, a different way of looking at AGN "downsizing". This is accompanied by a progressive steepening of the faint end slope of the LF (upper left panel): low-luminosity AGN become more and more dominant in the overall number density of AGN as time progresses.

Such a detailed view of the evolution of active galactic nuclei, with its distinctive signatures of "downsizing", has lent additional support to the notion that the lives of growing black holes must be intimately linked to those of their host galaxies. Indeed, both galaxies and black holes show signs of a similar differential evolution. The very term "downsizing" was first used by Cowie et al. (1996) to describe the finding that actively star-forming galaxies at low redshift have smaller masses than actively star-forming galaxies at $z \sim 1$. It has come to identify, in the current cosmology jargon, a variety of possibly distinct phenomena, not just related to the epoch of star formation, but also to that of star formation quenching, or galaxy assembly (see Chapter "Galaxies in the Cosmological Context" by G. De Lucia in this same volume). Our current understanding of AGN evolution, encapsulated in the observable evolution of their bolometric luminosity function, emphatically suggests that growing nuclear black holes take part in this global process of structure formation. 


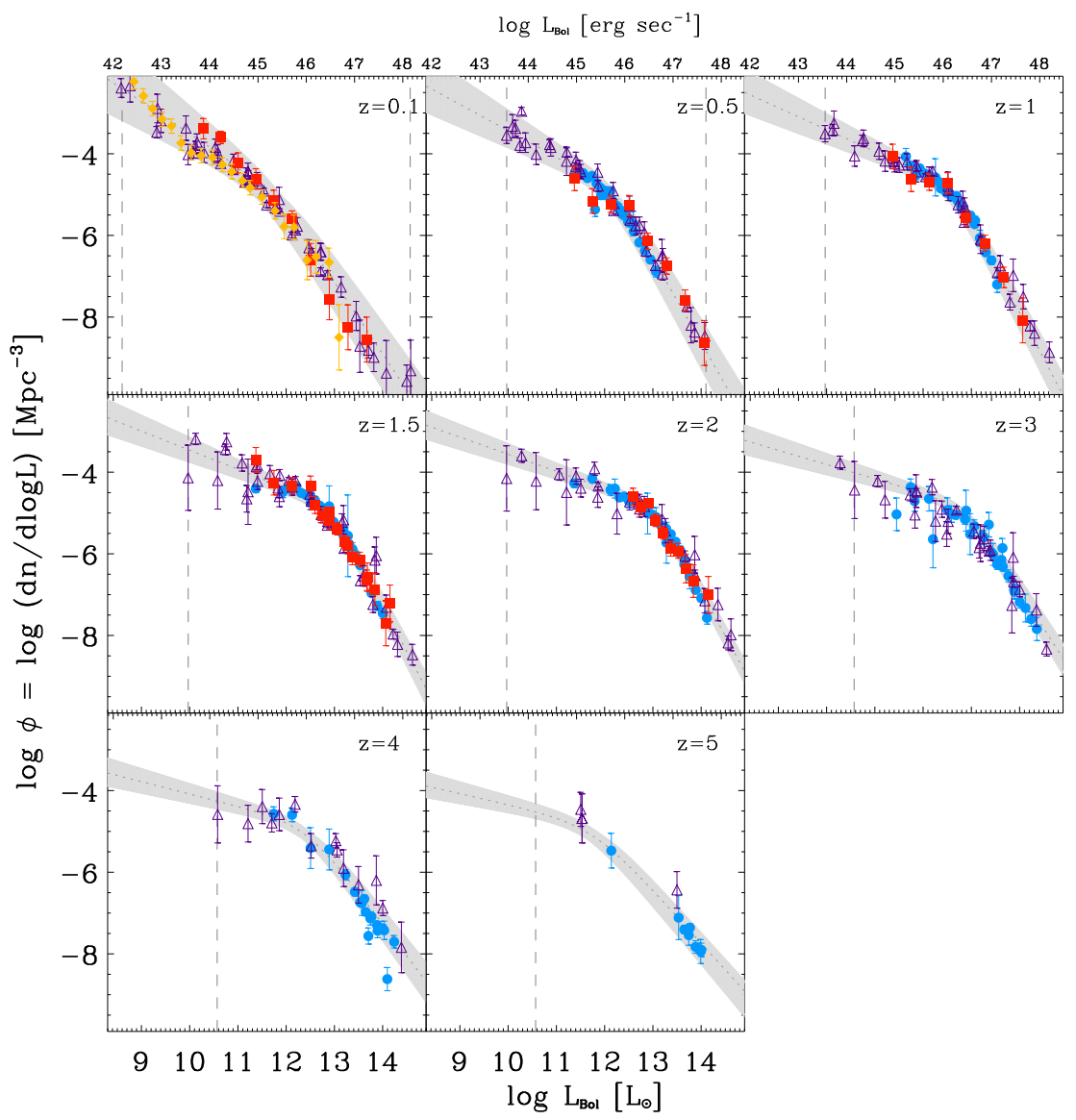

Figure 17: Bolometric AGN luminosity function (gray band) as a function of redshift, as calculated by Hopkins et al. (2007). The different symbols and colors refer to different bands from which data have been extracted: solid blue circles are optical data, filled red squares IR (at 15 $\mu \mathrm{m}$ ), blue and purple triangle are soft and hard X-ray data, respectively, and the filled orange diamonds are luminosities from emission lines. The vertical dashed lines bracket the observational limits. We refer the reader to Hopkins et al. (2007) for a more detailed description of the data and methodology used to extract the bolometric luminosity function. Data points courtesy of P. Hopkins 

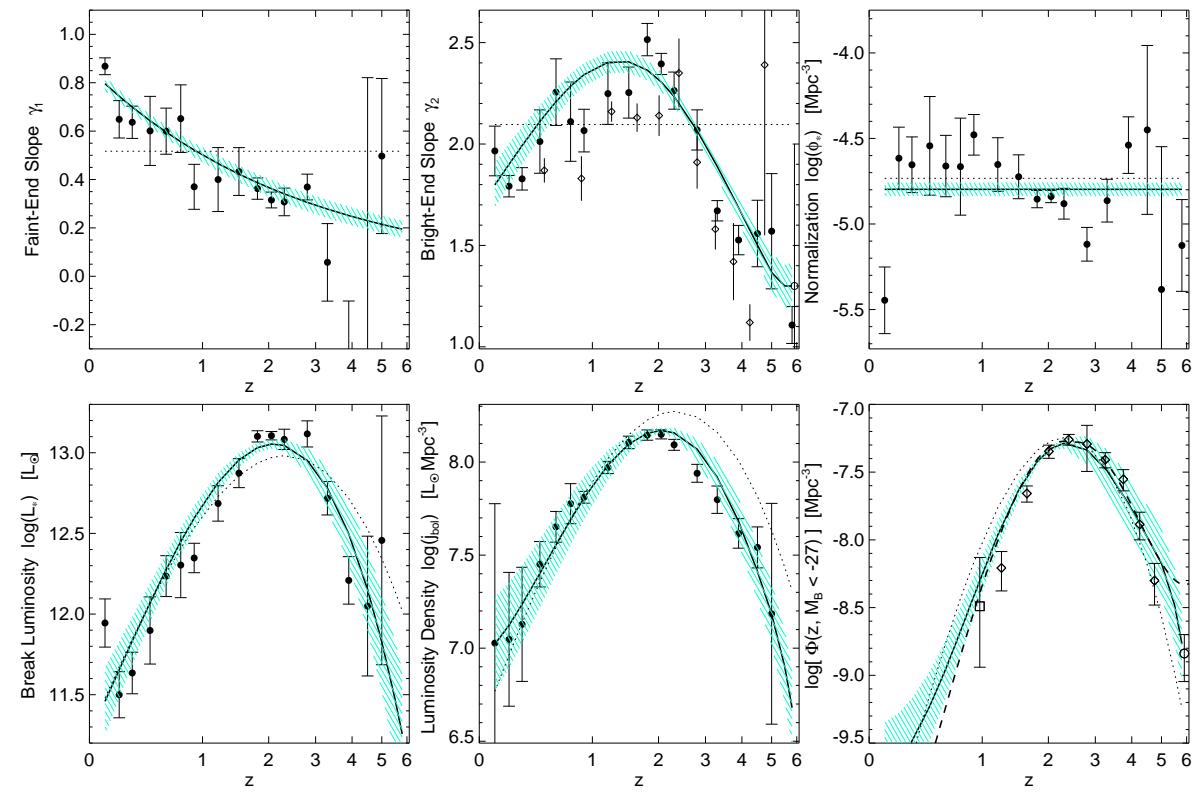

Figure 18: Best-fit AGN bolometric LF double power-law parameters as a function of redshift. Symbols show the best-fit values to data at each redshift, dotted lines the best-fit PLE model, and solid lines the best-fit full model (a luminosity and density evolution one). Although PLE is appropriate for a lowest order fit, both the bright- and faint-end slopes evolve with redshift to high significance. The bottom right panel shows the predicted number density of bright optical quasars from the full fit (solid line), compared to that observed ones. From Hopkins et al. (2007). 


\subsection{AGN Clustering and the Large scale structure of the universe}

The bolometric luminosity function of AGN provides the basic tool to describe the differential evolution of growing black holes. Some key properties of AGN, however, remain impossible to determine on the sole basis of the observed LF. As it is increasingly difficult to measure black hole masses at high redshift, and currently only possible for bright, un-obscured broad line QSOs, we do not have robust, direct, observational constraints on the distribution of AGN Eddington ratios beyond the local universe. The Eddington ratio distribution, in turn, depends on the details of the average AGN lightcurves which could reveal important details of the physical processes driving the accreting gas towards the black hole.

The spatial distribution of QSOs (clustering) in the sky could provide such an alternative method to estimate lifetimes (Martini \& Weinberg 2001; see also Chapter "Large scale structure of the universe" by A. Coil in this volume). In the current $\Lambda$ CDM paradigm for structure formation, more clustered objects are rarer and live in more massive dark matter structures (or "halos"). Thus, if AGN are strongly clustered, their hosts must be rare objects, too, and the effective AGN lifetime must be long, in order for such a rare "parent" population to account for the total AGN luminosity density observed. If, on the other hand, their clustering is comparable to the clustering of smaller, less massive, dark matter halos, their host are more common and their luminous phases must therefore have short duration.

A commonly used technique for measuring the spatial clustering of a class of objects is the two-point correlation function $\xi(r)$, which measures the excess probability $d P$ above a random distribution of finding an object in a volume element $d V$ at a distance $r$ from another randomly chosen object:

$$
d P=n[1+\xi(r)] d V
$$

where $n$ is the mean number density of objects. In the scale range between a few tens of kpc and a few tens of Mpc, for most classes of astronomical objects $\xi(r)$ can be described by a single power-law:

$$
\xi(r)=\left(\frac{r}{r_{0}}\right)^{-\gamma}
$$

where $r_{0}$ is the correlation, or clustering, length, defined as the scale at which the two-point correlation function is equal to unity.

Unfortunately, a direct comparison of the measured clustering length of AGN with that expected for dark matter halos of different masses is hampered by the fact that, according to current theories of structure formation, galaxies (and their nuclear black holes) do not follow the distribution of the underlying matter, but form in the high-density peaks of the dark matter field. The bias of any astrophysical population $X$ is defined as the (square root of the) ratio between the two-point autocorrelation functions of population $X$ and of the dark matter (DM) halos: $b_{\mathrm{X}, \mathrm{DM}}(r) \equiv \sqrt{\xi_{\mathrm{X}}(r) / \xi_{\mathrm{DM}}(r)}$.

Many groups have now been able to measure the clustering of AGN at different luminosities, bands, scales and redshifts (see, e.g., the recent review of Shankar 2009 for a complete list of references). Overall, the clustering length of quasars appears to be an increasing function of redshift, but does not depend strongly on luminosity.

As shown in Fig. 19, the bias of optically selected (broad line) AGN increases with redshift following an evolution at approximately constant dark matter halo mass (since halos of a fixed 
mass are progressively more clustered towards higher redshift), in the range $\log M_{\mathrm{DM}} \simeq 12.5 \div$ $13 \mathrm{~h}^{-1} M_{\odot}$ at redshifts $z<3.5$.

Instead, X-ray selected objects (Allevato et al., 2011), both obscured and unobscured, reside in more massive DM structures at all redshifts $z<2.25$, with a typical mass of the hosting halos constant over time in the range $\log M_{\mathrm{DM}} \simeq 13 \div 13.5 \mathrm{~h}^{-1} M_{\odot}$.

By combining the number density of AGN with that of the hosting dark matter halos, one can estimate an AGN duty cycle, and a corresponding average lifetime. The observed biases of the rare, luminous broad lined quasars imply timescales of the order of $10^{7} \div 10^{8}$ years, increasing with redshift, as the massive halos typically hosting AGN become increasingly rare.

For X-ray selected AGN, a larger duty cycle is inferred, which translates into an AGN lifetime of $\sim 0.1 \div 1 \mathrm{Gyr}$, about one order of magnitude longer than that estimated for optically bright QSOs at the same redshift. This is mainly due to the higher number density and higher bias of AGN found in X-ray selected samples.

Numerical simulations of merger-induced AGN activity in a cosmological context have shown (Bonoli et al., 2009) that the clustering of optically selected quasars is well explained by a model in which these objects are triggered by major merger events. The difference between optically selected and the (lower-luminosity) X-ray selected AGN of Figure 19 might suggest that X-ray selected AGN are triggered by different (secular) processes which may be capable of fueling luminous AGN in the gas-rich environment of star-forming galaxies at high redshift. The same models also predict an increase in AGN duty cycle for the brightest quasars at halo masses larger than $10^{12} M_{\odot}$, but fail to reproduce the large biases for less luminous X-ray selected AGN at $z \sim 1$, possibly pointing (again) towards the need for a larger variety of AGN triggering mechanisms for this class of objects.

\section{Cosmology I: The growth of supermassive black holes in galax- ies}

As we have discussed in the previous section, the strong cosmological evolution of the quasar population was recognized early on by observers in essentially all bands of the electromagnetic spectrum. In the early 1990s, deep optical surveys of star-forming galaxies began to probe the cosmological evolution of the rate at which stars are formed within galaxies, thus providing robust constraints for models of galaxy formation and evolution (the so-called Lilly-Madau plot; Madau et al. 1996). It was soon clear that QSOs luminosity density and Star Formation Rate (SFR) density evolved in similar fashion, being much higher in the past, with a possible (very broad) peak at $z \approx 2$ (Boyle \& Terlevich, 1998).

In the previous section we have traced the history of the study of AGN luminosity functions in various spectral bands, closing with an assessment of our current understanding of the bolometric luminosity function evolution. A reliable census of the bolometric energy output of growing supermassive black holes (see, e.g., the central bottom panel of Figure 18) allows a more direct estimate of the global rate of mass assembly in AGN, and an interesting comparison with that of stars in galaxies. Together with the tighter constraints on the "relic" SMBH mass density in the local universe, $\rho_{\mathrm{BH}, 0}$, provided by careful application of the scaling relations between black hole masses and host spheroids, this enables meaningful tests of the classical 'Soltan argument' (Soltan, 1982), according to which the local mass budget of black holes in galactic nuclei should 


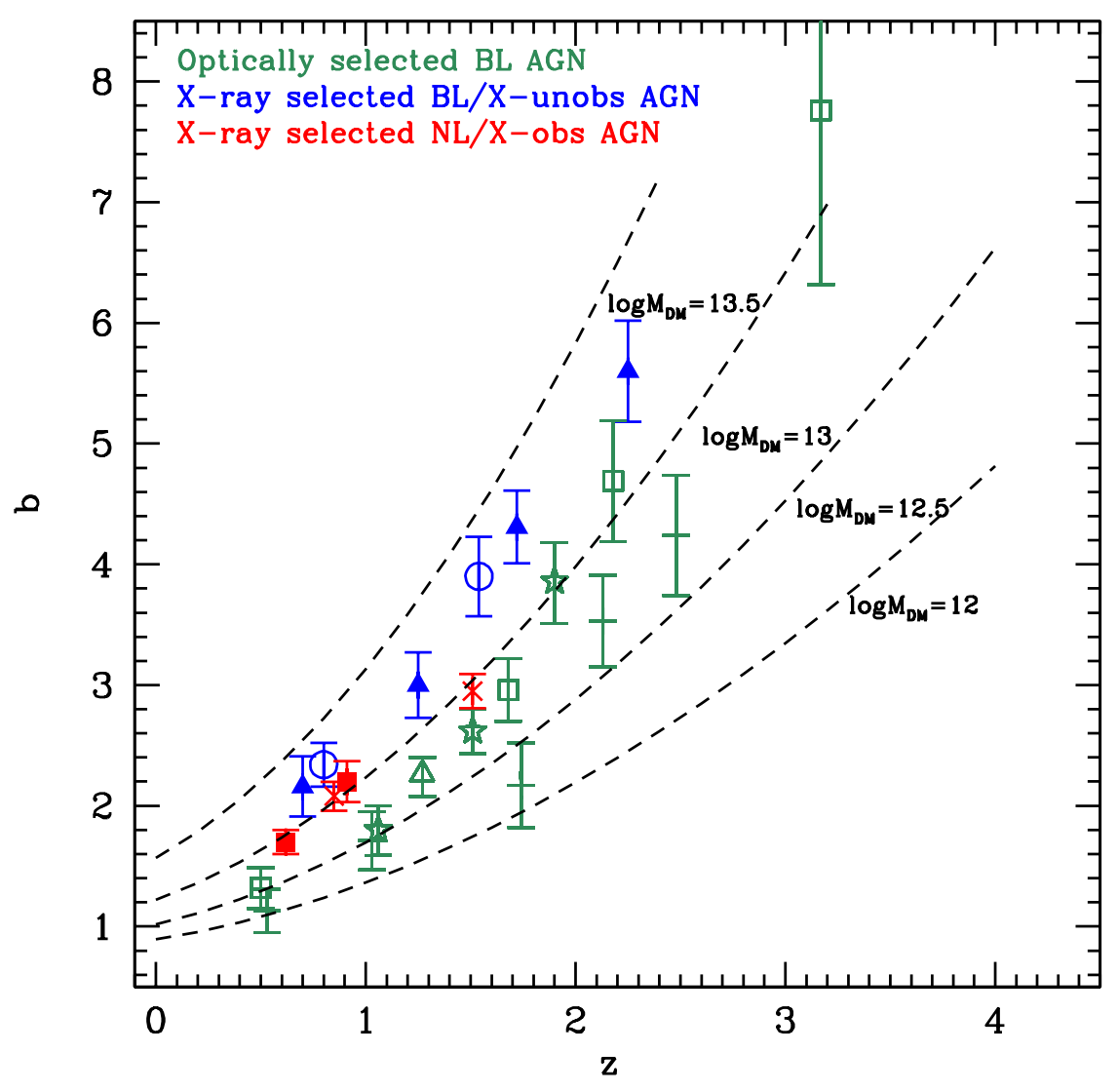

Figure 19: Bias parameter as a function of redshift for various AGN surveys. The range of the bolometric luminosity probed is given in parenthesis: (i) optically selected BL AGN: greencrosses $\left(2 \mathrm{dF} ; 45.3<\log L_{\text {bol }}<46.7\right)$, green-stars $\left(2 \mathrm{dF} ; 45.5<\log L_{\text {bol }}<47.4\right)$, green-open squares (SDSS; $45.6<\log L_{\mathrm{bol}}<46.9$ ) and green-open triangles (SDSS; $\log L_{\mathrm{bol}} \sim 46.5$ ); (ii) X-ray selected unobscured AGN: blue triangles and blue open-circles (XMM-COSMOS; $45<$ $\log L_{\text {bol }}<45.7$ ) and (iii) X-ray selected obscured AGN: red squares and red crosses (XMMCOSMOS; $44.1<\log L_{\mathrm{bol}}<44.6$ ). See Allevato et al. (2011) for a similar figure and the full list of references for the observed data points. The dashed lines show the expected bias evolution of typical DM halo masses. 
be accounted for by integrating the overall energy density released by AGN, with an appropriate mass-to-energy conversion efficiency.

Many authors have carried out such a calculation, either using the CXRB as a "bolometer" to derive the total energy density released by the accretion process (Fabian \& Iwasawa, 1999), or by considering evolving AGN luminosity functions (Yu \& Tremaine, 2002; Marconi et al., 2004; Merloni \& Heinz, 2008). Despite some tension among the published results that can be traced back to the particular choice of AGN LF and/or scaling relation assumed to derive the local mass density, it is fair to say that this approach represents a major success of the standard paradigm of accreting black holes as AGN power-sources, as the radiative efficiencies needed to explain the relic population are within the range $\approx 0.06 \div 0.20$, predicted by standard relativistic accretion disc theory (Novikov \& Thorne, 1973).

In this section, we begin with a schematic account of the current constraints on the black hole mass density growth, and discuss some recent attempts to compare it on a quantitative level with the observed growth of the galaxy population. This will be followed by the (related) discussion of the possible evolution of the scaling relations.

\subsection{A global view of the accretion history of the universe}

Under the standard assumption that black holes grow mainly by accretion, their cosmic evolution can be calculated from the bolometric luminosity function of AGN $\phi\left(L_{\mathrm{bol}}, z\right)$, where $L_{\mathrm{bol}}=$ $\epsilon_{\mathrm{rad}} \dot{M} c^{2}$ is the bolometric luminosity produced by a SMBH accreting at a rate of $\dot{M}$ with a radiative efficiency $\epsilon_{\mathrm{rad}}$. The non-negligible fraction of the AGN population which is unaccounted for in current surveys, the so-called Compton thick AGN (see $\$ 2.1 .2$ above), is usually included in the bolometric luminosity function by assuming a redshift-invariant column density distribution as measured in the very local universe and an overall number density of heavily obscured AGN that fits the CXRB.

The total, integrated mass density in supermassive black holes can then be computed as a function of redshift:

$$
\frac{\rho_{\mathrm{BH}}(z)}{\rho_{\mathrm{BH}, 0}}=1-\int_{0}^{z} \frac{\Psi_{\mathrm{BH}}\left(z^{\prime}\right)}{\rho_{\mathrm{BH}, 0}} \frac{d t}{d z^{\prime}} d z^{\prime}
$$

where the black hole accretion rate (BHAR) density is given by:

$$
\Psi_{\mathrm{BH}}(z)=\int_{0}^{\infty} \frac{\left(1-\epsilon_{\mathrm{rad}}\right) L_{\mathrm{bol}}}{\epsilon_{\mathrm{rad}} c^{2}} \phi\left(L_{\mathrm{bol}}, z\right) d L_{\mathrm{bol}}
$$

and

$$
\frac{d t}{d z}=-\left[(1+z) H_{0} \sqrt{(1+z)^{3} \Omega_{m}+\Omega_{\Lambda}}\right]^{-1}
$$

The exact shape of $\rho_{\mathrm{BH}}(z)$ and $\Psi_{\mathrm{BH}}(z)$ then depends only on the local black hole mass density $\rho_{\mathrm{BH}, 0}$ and the (average) radiative efficiency $\epsilon_{\mathrm{rad}}$.

We can then link the growth of SMBH from eq. (6) to the growth of stellar mass in galaxies. To do so, we will use the Hopkins et al. (2007) bolometric LF of AGN (see section 2.1.4 above) 4 .

\footnotetext{
${ }^{4}$ As discussed in Marconi et al. (2004), in order to correctly estimate the total bolometric output of an AGN, care should be taken in avoiding double counting of the IR reprocessed emission. This appears not have been done in Hopkins et al. (2007), so we correct the bolometric luminosities by $30 \%$ to account for this.
} 

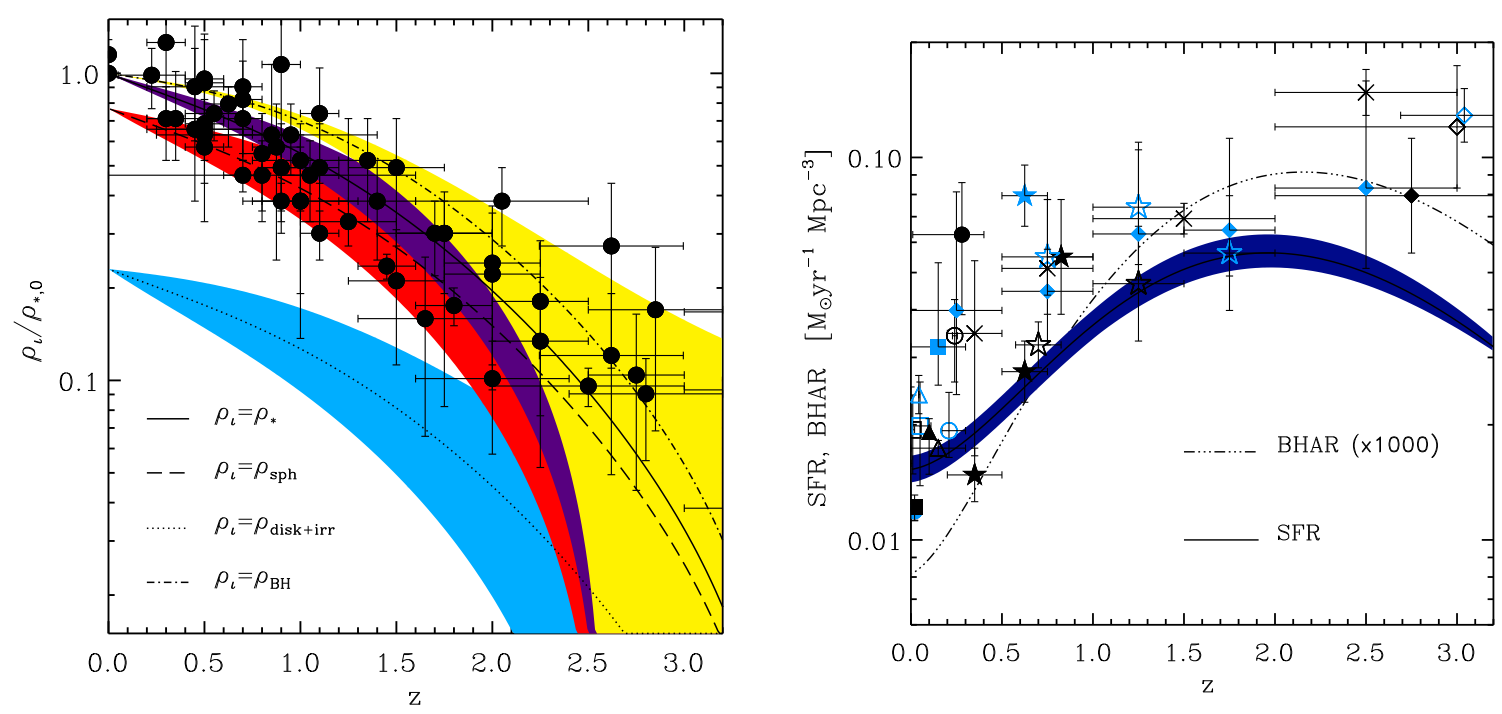

Figure 20: Left: Evolution of the stellar mass density as a function of redshift (black points, observations), where the density is given as a ratio to the local value, $\rho_{*, 0}=5.6 \times 10^{8} M_{\odot} \mathrm{Mpc}^{-3}$ (Cole et al., 2001). Shaded areas represent 1-sigma confidence intervals of the model fits. Solid black line with purple shaded area shows the best joint fit from eq. (9) and eq. (10) to both stellar mass and SFR density points (left and right panels), while the dot-dashed line with yellow shaded area marks the normalized evolution of the SMBH mass density only. The slight offset between the two is compensated by a change in the normalization of the average black hole to spheroid mass ratio with redshift (see text for details). The dashed (dotted) line with red (blue) shaded area shows the relative growth of the mass density in spheroids (discs). Values of $\lambda_{0}=0.3$ (Fukugita \& Peebles, 2004) and $\rho_{\mathrm{BH}, 0}=4.2 \times 10^{5} \mathrm{M}_{\odot} \mathrm{Mpc}^{-3}$ (Shankar, 2009) are adopted here. Right: The corresponding best-fit relation for the SFR density evolution, from eq. (10) is shown with a solid line and dark blue shaded area. The dash-triple-dotted line is (1000 times) the black hole accretion rate density $\Psi_{\mathrm{BH}}(z)$ (BHAR). It appears that the BHAR declines slightly faster than the SFR, another way to emphasize the need of an evolution in the average $M_{\mathrm{BH}} / M_{\mathrm{sph}}$ ratio. 
Because local SMBH are observed to correlate with spheroids only, we introduce the parameter $\lambda(z)$, the ratio of the mass in disks and irregulars to that in spheroids at any redshift, so that the total stellar mass density can be expressed as: $\rho_{*}(z)=\rho_{\mathrm{sph}}(z)+\rho_{\text {disk }+\operatorname{irr}}(z)=\rho_{\mathrm{sph}}(z)[1+\lambda(z)]$.

We can now assume that $\lambda(z)$ evolves according to $\lambda(z)=\lambda_{0}(1+z)^{-\beta}$, where $\lambda_{0}$ is the value of the disk to spheroid mass density ratio in the local universe. Also, we assume that the mass density of spheroids and supermassive black holes evolve in parallel, modulo a factor $(1+z)^{-\alpha}$, obtaining a prediction for the observable stellar mass density evolution as traced by SMBH growth:

$$
\rho_{*}(z)=\mathcal{A}_{0} \rho_{\mathrm{BH}}\left(\epsilon_{\mathrm{rad}}, z\right)(1+z)^{-\alpha}\left[1+\lambda_{0}(1+z)^{-\beta}\right]
$$

where $\mathcal{A}_{0}$ is the constant of proportionality in the SMBH mass-spheroid mass relation. By taking the derivative of eq. (9), accounting for stellar mass loss, an expression is also found for the corresponding star formation rate (SFR) density evolution:

$$
d \rho_{*}(z) / d t=\Psi_{*}(z)-\int_{z_{i}}^{z} \Psi_{*}\left(z^{\prime}\right) \frac{d \chi\left[\Delta t\left(z^{\prime}-z\right)\right]}{d t} \frac{d t}{d z^{\prime}} d z^{\prime},
$$

where $\chi\left[\Delta t\left(z^{\prime}-z\right)\right]$ is the fractional mass loss that a simple stellar population experiences after a time $\Delta t$ (corresponding to the redshift interval $\left(z^{\prime}-z\right) \sqrt{5}$ and $z_{i}$ is the redshift of (instantaneous) formation of the first stellar populations; in practical terms, fixing any $z_{i}>4$ will not substantially alter the results of such kind of computations.

With these expressions we fit observational data points of both $\rho_{*}(z)$ and $\operatorname{SFR}(z)$. For each choice of $\rho_{\mathrm{BH}, 0}, \lambda_{0}$, and of the critical accretion rate $\dot{m}_{\mathrm{cr}}$, the fitting functions depend only on three parameters: $\alpha, \beta$ and the radiative efficiency $\epsilon_{\mathrm{rad}}$. One example of such fits is shown in Fig. 20 for the specific case $\rho_{\mathrm{BH}, 0}=4.2 \times 10^{5} M_{\odot} \mathrm{Mpc}^{-3}$ (Shankar, 2009), and $\lambda_{0}=0.3$ (Fukugita \& Peebles, 2004).

Because the drop in the AGN integrated luminosity density at low $z$ is apparently faster than that in the SFR density, the average black hole to spheroid mass ratio must evolve (slightly) with lookback time $(\alpha>0)$. This result is independent of the local black hole mass density, and independent of $\lambda_{0}$. For the particular example shown here, the average radiative efficiency turns out to be $\epsilon_{\mathrm{rad}}=0.08_{-0.02}^{+0.01}$, while we obtain $\alpha=0.35_{-0.3}^{+0.22}$ (both shown with $3-\sigma$ confidence bounds). At face value, this would imply a very mild evolution of the average $M_{\mathrm{BH}} / M_{\mathrm{sph}}$ mass ratio. We will discuss in section 3.2 .1 how these constraints compare with recent efforts to directly measure the ratio of black hole to host galaxy mass at high redshift.

This simple exercise should make clear that the available constraints on SMBH growth from the observed bolometric LF are robust enough to provide interesting non trivial insight into the cosmological co-evolution of AGN and galaxies.

\subsubsection{The Evolution of the SMBH mass function}

Despite the relative successes of 'Soltan argument'-like calculations of the integral evolution of the SMBH mass density, it is obvious that a much greater amount of information is contained in the differential distributions (mass and luminosity functions). We will now discuss attempts to use this information to constrain the evolution of the mass function of SMBH.

\footnotetext{
${ }^{5}$ An analogous term for $\rho_{\mathrm{BH}}$, due to the ejection of SMBHs from galaxy halos after a merger event, is much more difficult to estimate and is neglected here.
} 
As opposed to the case of galaxies, where the direct relationship between the evolving mass functions of the various morphological types and the distribution of star forming galaxies is not straightforward due to the never-ending morphological and photometric transformation of the different populations, the situation in the case of SMBH is much simpler. For the latter case, we can assume their evolution is governed by a continuity equation (Merloni \& Heinz, 2008, and references therein), where the mass function of $\mathrm{SMBH}$ at any given time can be used to predict the mass function at any other time, provided the distribution of accretion rates as a function of black hole mass is known. Such an equation can be written as:

$$
\frac{\partial \psi(\mu, t)}{\partial t}+\frac{\partial}{\partial \mu}(\psi(\mu, t)\langle\dot{M}(\mu, t)\rangle)=0
$$

where $\mu=\log M$ is the black hole mass in solar units, and $\psi(\mu, t)$ is the SMBH mass function at time $t .\langle\dot{M}(\mu, t)\rangle$ is the average accretion rate of SMBH of mass $M$ at time $t$ and can be defined through a "fueling" function, $F(\dot{\mu}, \mu, t)$, which describes the distribution of accretion rates for objects of mass $M$ at time $t$

$$
\langle\dot{M}(\mu, t)\rangle=\int \dot{M} F(\dot{\mu}, \mu, t) \mathrm{d} \dot{\mu}
$$

Such a fueling function is not a priori known, and observational determinations thereof have been possible in any robust sense only for the extremes of the overall population. However, the AGN fueling function can be derived by inverting the integral equation that relates the luminosity function of the population in question with its mass function. And so, we can write:

$$
\phi(\ell, t)=\int F(\ell-\zeta, \mu, t) \psi(\mu, t) \mathrm{d} \mu
$$

with the definitions $\ell \equiv \log L_{\mathrm{bol}}$ and $\zeta \equiv \log \left(\epsilon_{\mathrm{rad}} c^{2}\right)$, with $\epsilon_{\mathrm{rad}}$ the radiative efficiency, here assumed to be constant.

Using this approach, eq. (11) can be integrated backwards from $z=0$, where we have simultaneous knowledge of both the mass function, $\psi(\mu)$, and the luminosity function, $\phi(\ell)$, thus evolving the SMBH mass function backwards in time, up to where (i) reliable estimates of the AGN luminosity functions are available, and (ii) the accumulated error in the mass function becomes of the order of the mass function itself.

The first thing to notice from such an approach is that the different shapes of the observed SMBH mass function $\psi(\mu)$ (that decays exponentially at high masses) and AGN LF $\phi(\ell)$ (well described by a double power-law) necessitate a broad distribution of accretion rate (Merloni \& Heinz, 2008): AGN, as a population, cannot be simply characterized by an on-off switch at fixed Eddington ratio. Instead, integration of eq. (11) gives insight on the relative importance of massive black hole growth at different accretion rates.

Figure 21] shows the number density evolution as a function of redshift for black holes of constant mass (in the range $8<\log \left(M_{\mathrm{BH}} / M_{\odot}\right)<8.5$ ) at different Eddington ratios. Numerically, the AGN population is always dominated by slowly accreting objects, but the observed flattening of the bolometric LF shape (see Fig. 18) implies that the relative number of rapidly accreting black holes increases significantly with redshift. In terms of grown mass, however, high-Eddington-ratio AGN strongly dominate the budget, as shown in the top panel of Fig. 21 . most of the mass of a typical $\approx 10^{8} M_{\odot}$ black hole has been accumulated in (short-lived) episodes of rapid accretion, between a few and a few tens of per cent of the Eddington luminosity. 


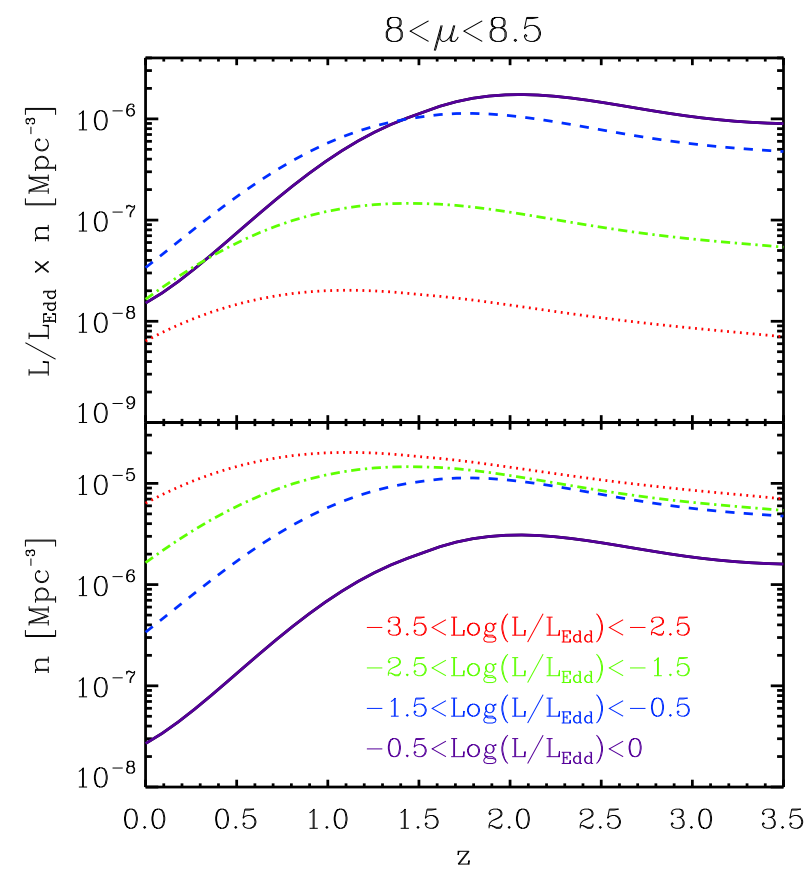

Figure 21: Bottom: Evolution of the number density (objects per comoving Mpc) as a function of redshift for black holes of constant mass (in the range $8<\log \left(M_{\mathrm{BH}} / M_{\odot}\right)<8.5$ ) at different Eddington ratios. Solid (purple), dashed (blue), dot-dashed (green) and dotted (red) lines correspond to intervals of Eddington ratio ranging from 1 to $10^{-3.5}$. Top: The product of average Eddington ratio times number density vs. redshift. Despite being numerically subdominant, rapidly accreting black holes (i.e., those with $L / L_{\mathrm{Edd}}>3 \%$ ) clearly dominate the mass assembly of SMBH in this range of masses. 

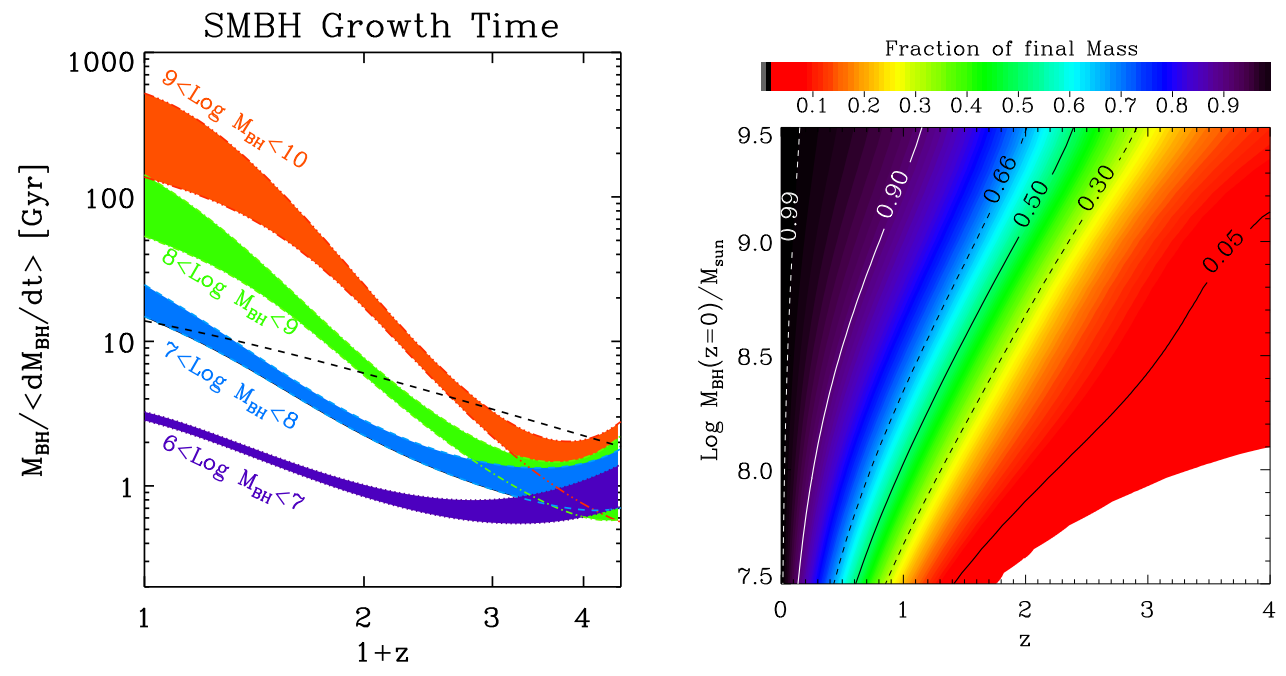

Figure 22: Left: Average Growth time of Supermassive Black Holes (in years) as a function of redshift for different black hole mass ranges. The dashed line marks the age of the universe; only black holes with instantaneous growth time smaller than the age of the universe at any particular redshift can be said to be effectively growing. Right: the fraction of the final black hole mass accumulated as a function of redshift and final (i.e. at $z=0$ ) mass is plotted as contours.

The specific instantaneous ratio of black hole mass to accretion rate as a function of SMBH mass defines a timescale, the so-called growth time, or mass doubling time. The redshift evolution of the growth time distribution can be used to identify the epochs when black holes of different sizes grew the largest fraction of their mass: black holes with growth times longer than the age of the universe are not experiencing a major growth phase, which must have necessarily happened at earlier times.

Figure 22 (left) shows that, according to this simple estimate, while at $z<1$ only black holes with masses smaller than $10^{7} M_{\odot}$ are experiencing significant growth, as we approach the peak of the black hole accretion rate density $(z \sim 1.5-2)$, we witness the rapid growth of the entire SMBH population.

Solutions of the continuity equation also allow one to trace the growth of black holes of a given final (i.e., at $z=0$ ) mass. The right hand side panel of Fig. 22 shows that, for the most massive black holes $\left(>10^{9} M_{\odot}\right)$, half of the mass was already in place at $z \sim 2$, while those with $M(z=0)<10^{8} M_{\odot}$ had to wait until $z \sim 1$ to accumulate the same fraction of their final mass.

\subsection{The AGN-galaxy connection}

The very existence of scaling relations between black holes and their host galaxies, and the broad accretion rate distributions of AGN derived from the continuity equation approach imply that, as observed throughout the electromagnetic spectrum, growing black holes will display a large range of "contrast" with the host galaxy light. 
The most luminous QSO, accreting at the highest Eddington ratios, will be able to outshine the stellar light from the galaxy, while less luminous, Seyfert-like AGN will have a global SED with a non-negligible contribution from the host (see also eq. 3 above). At high redshift, when it becomes increasingly difficult to spatially separate the nuclear emission, unbiased AGN samples will have optical-NIR colors spanning a large range of intermediate possibilities.

Figure 23 nicely illustrates this point. It is taken from the analysis of an X-ray selected sample of AGN in the COSMOS field (Brusa et al., 2010), the largest fully identified and redshift complete AGN sample to date. It displays the slope of the rest-frame SED in the optical $\left(\alpha_{\mathrm{OPT}}\right.$, between 0.3 and $1 \mu \mathrm{m}$ ) and NIR ( $\alpha_{\mathrm{NIR}}$ between 1 and $\left.3 \mu \mathrm{m}\right)$. Pure QSOs, i.e., objects in which the overall SED is dominated by the nuclear (AGN) emission have a typical dip in the NIR region, and would lie close to the empty blue star in the lower right corner (positive optical slope and negative NIR slope). The location of the X-ray selected AGN in Figure 23 shows instead that, in order to describe the bulk of the population, one needs to consider both the effects of obscuration (moving each pure QSO in the direction of the orange arrow) and an increasing contribution from galactic stellar light (moving the objects towards the black stars in the upper part of the diagram).

This demonstrates that current multi-wavelength extragalactic surveys are sensitive enough to disentangle the complex interplay between nuclear and galaxy light in the SED of more typical AGN. It is no coincidence that such surveys are beginning to probe the details of the co-evolution of black holes and host galaxies on an object-by-object basis. In the following section we will briefly discuss how one can use such information to observationally trace the evolution of the scaling relations between nuclear SMBH and their host galaxies.

\subsubsection{Redshift evolution of the scaling relations}

Local scaling relations between black hole mass and structural properties of their (spheroidal) hosts have been unable to unambiguously determine the physical nature of the SMBH-galaxy coupling. A large number of theoretical models for the AGN-galaxy interaction responsible for establishing, for example, the $M-\sigma_{*}$ relation, have been proposed, all tuned to reproduce the $z=0$ observations. One obvious way out of this impasse is the study of their evolution.

In recent years, a number of groups have employed different techniques to detect signs of evolution in any of the locally observed scaling relations. Only type 1 AGN, with un-obscured broad line region allow a simple direct estimate of $\mathrm{BH}$ masses, via the so-called "virial" or empirically calibrated "photo-ionization" method (Peterson et al., 2004). Based on existing samples of broad line QSOs, most efforts have been devoted to the study of the $M_{\mathrm{BH}}-\sigma_{*}$ relation. For example, Salviander et al. (2007) have used narrow nebular emission lines ([OIII], [OII]) excited by the AGN emission in the nuclear region of galaxies as proxies for the central velocity dispersion, and compared these to the black hole mass estimated from the broad line width of QSOs from $z \sim 0$ to $z \sim 1$. In this case, a large scatter has been found in the relation between $M_{\mathrm{BH}}$ and $\sigma_{*}$.

An alternative path is to study carefully selected samples of moderately bright AGN in narrow redshift ranges, where the host's stellar velocity dispersion can be measured directly from the absorption lines in high signal-to-noise spectra. These studies also found evidence of (strong) positive evolution of the $M_{\mathrm{BH}}$ to $\sigma_{*}$ ratio compared to the local value (see Bennert et al. 2011, and references therein). This method, although promising and reliable, is quite inefficient and 


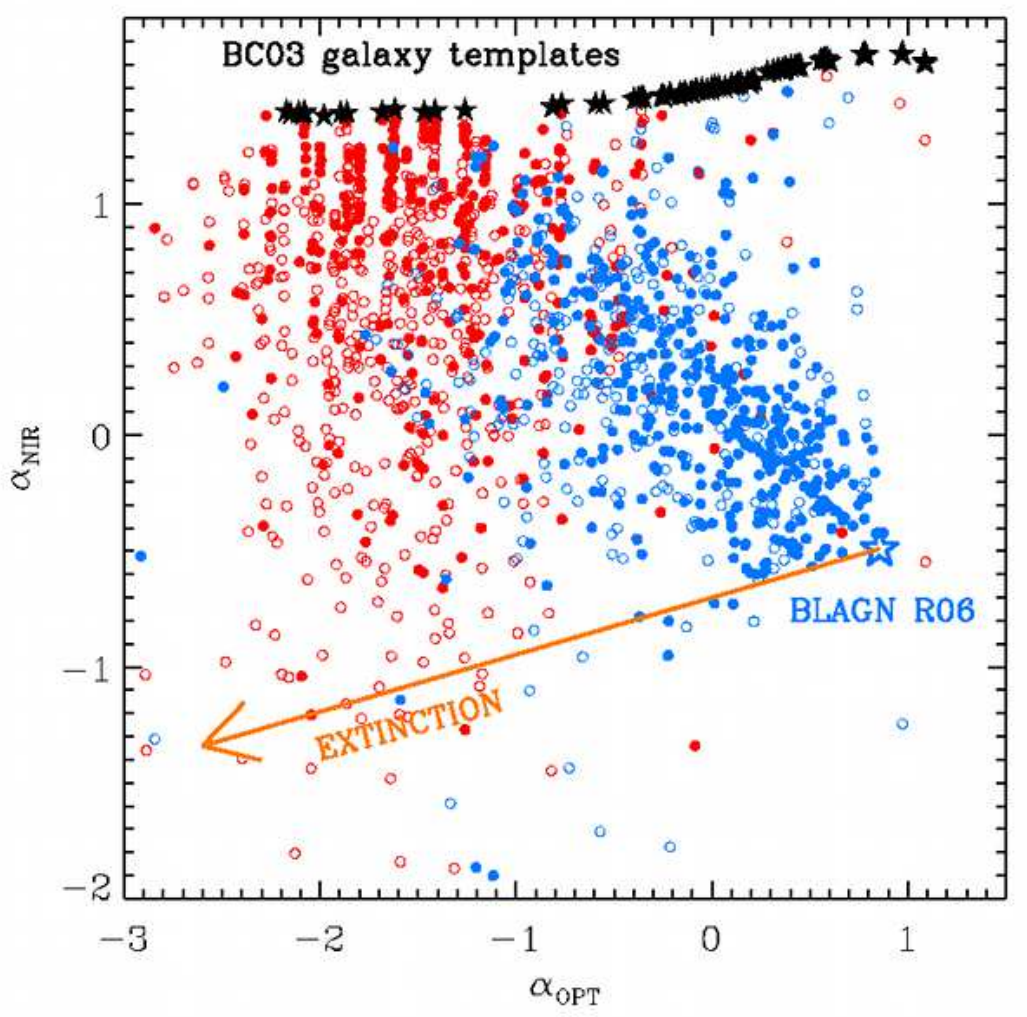

Figure 23: Observed rest-frame SED slopes in the optical ( $\alpha_{\mathrm{OPT}}$, between 0.3 and $\left.1 \mu \mathrm{m}\right)$ and NIR ( $\alpha_{\text {NIR }}$ between 1 and $3 \mu \mathrm{m}$ ) for all ( 1650) X-ray selected AGN in the COSMOS survey. Blue filled circles denote spectroscopically confirmed type 1 (Broad Lined) AGN, blue empty circles denote candidate type 1 AGN from the photo-z sample. Red filled circles are spectroscopically confirmed type 2 (Narrow lined) AGN, empty red circles are candidate type 2 AGN from the photo-z sample. The empty blue star marks the colors of a pure intrinsic type 1 quasar SED (from Richards et al. 2006), while black stars are the loci of synthetic spectral templates of galaxies, with increasing levels of star formation form the left to the right. Nuclear obscuration, parametrized with a Calzetti extinction law, moves every pure type 1 AGN along the direction of the orange arrow. 

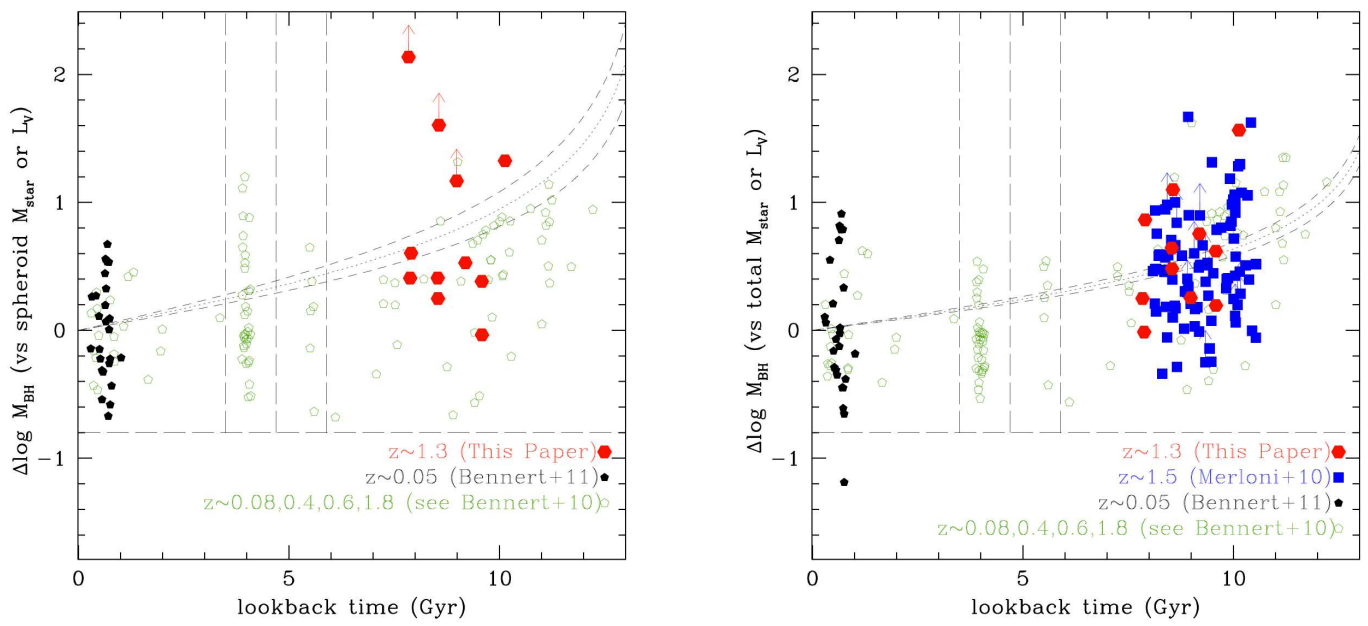

Figure 24: Left: Offset in $\log M_{\mathrm{BH}}$ as a function of constant spheroid host galaxy mass (red filled pentagons) with respect to the fiducial local relation of AGNs (black filled circles). The offset as a function of constant stellar spheroid luminosity is over-plotted (green open symbols), corresponding to AGNs at different redshifts. The best linear fit derived here is over-plotted as dotted line $M_{\mathrm{BH}} / M_{\mathrm{sph}} \propto(1+z)^{2.1 \pm 0.3}$; dashed lines: $1 \sigma$ range. Right: The same as in the left panel as an offset in $\log M_{\mathrm{BH}}$ as a function of constant total host galaxy mass. The lines correspond to $M_{\mathrm{BH}} / M_{\text {host }} \propto(1+z)^{1.41 \pm 0.12}$. From Bennert et al. (2011), where a comprehensive list of references for the observational data points can also be found.

telescope-time consuming: secure detection of spectral absorption features in massive ellipticals at $1<z<2$ require hundreds of hours of integration time on an 8-meter class telescope.

When a good sampling of the AGN SED is instead available, rather than high-resolution, high signal-to-noise spectra, it is possible to try to decompose the overall spectral energy distribution into a nuclear and a galaxy component, and derive in this way the physical properties of the host galaxies of un-obscured AGN whose SMBH masses can be estimated from their broad lines (Merloni et al., 2010).

Other groups have chosen to try to derive information on the host mass of broad line AGN using multi-color image decomposition techniques. Due to the severe surface brightness dimming effects, employing these techniques for high-redshift QSOs becomes increasingly challenging, unless gravitationally lensed QSOs are selected. In all cases, very deep, high resolution optical images $(H S T)$ are necessary to reliably disentangle the nuclear from the host galaxy emission.

The main result of these various investigations is that our estimates of the type- 1 AGN host physical parameters are inconsistent with the hypothesis that they lie on the $z=0$ scaling relation (see Figure 24). At high redshift, bigger black holes are hosted in galaxies of a given mass as compared to what we observe locally. The best linear fit to the ensemble of observations shown in Figure 24 is $M_{\mathrm{BH}} / M_{\mathrm{sph}} \propto(1+z)^{2.1 \pm 0.3}$ for the black-hole-to-spheroid mass ratio and $M_{\mathrm{BH}} / M_{\text {host }} \propto(1+z)^{1.41 \pm 0.12}$ for the black-hole-to-total host stellar mass ratio.

However, the objects for which this study can be made are selected essentially on the basis of the nuclear (AGN) luminosity, and on the detectability of broad emission lines, clearly leading 
to a bias towards more massive black holes, similar to Malmquist bias for luminosity selected samples of standard candles. Can such a bias be responsible for the observed trends?

Let us consider in detail the effects on the observed systems of a given intrinsic scatter, $\sigma_{\mu}$, in the $M_{\mathrm{BH}}-M_{*}$ scaling relation. Any non-zero $\sigma_{\mu}$ implies that there is a range of possible masses $\log M_{*} \pm \sigma_{\mu}$ for each object of a given black hole mass $M_{\mathrm{BH}}$, where we have assumed, for simplicity, a symmetric scatter in the relation. If the number density of galaxies is falling off rapidly in the interval $\log M_{*} \pm \sigma_{\mu}$, it will then be more likely to find one of the more numerous small mass galaxies associated with the given black hole, and therefore a larger ratio $M_{\mathrm{BH}} / M_{*}$. Thus, given a distribution of galaxy masses, and provided that the scatter $\sigma_{\mu}$ is not too large, the logarithmic offset of each point from the correlation, assumed to be held fixed to the local determination, is given by:

$$
\Delta \log \left(M_{\mathrm{BH}} / M_{*}\right)=0.67 \times \Delta \log M_{\mathrm{BH}} \approx \sigma_{\mu}^{2}\left(\frac{d \log \phi}{d \log M_{*}}\right)_{\log M_{*}=(\mu-A) / B}
$$

where $\mu \equiv \log M_{\mathrm{BH}}$ and $(A, B)=(1.12,-4.12)$ are slope and intercept of the local scaling relation between $\mathrm{BH}$ and host galaxy masses (Häring \& Rix, 2004).

One can estimate observationally the logarithmic derivative of the galaxy mass function $\frac{d \log \phi}{d \log M_{*}}$. At $z \approx 2$, the offset expected from such a bias is of the order of $0.25 \operatorname{dex}$, if $\sigma_{\mu}=0.5$ and increases to about 0.5 dex for $\sigma_{\mu}=0.7$. The average offset shown in Fig. 24 is clearly in excess of what is expected in the most extreme case of large intrinsic scatter in the local relation, estimated to be less than 0.5 dex (Gültekin et al., 2009). The data point towards an evolution of the scaling relation, either in normalization or in scatter (or a combination of both).

What are the implications of these findings for our understanding of the cosmological coevolution of black holes and galaxies?

In the next section, we will discuss in more detail a number of physical processes by which AGN can regulate the growth of their host galaxies, thereby affecting any observable evolution of the scaling relations. We will see how, from the physical point of view, a clear distinction has to be made between two modes of AGN feedback.

The first one is associated to the numerous, long-lived, LLAGN, with emitted power dominated by the kinetic energy of their jets and outflows. It becomes increasingly important for very massive holes at low redshift (see section 3.1.1 above). Many models of galaxy formation invoke such a feedback mechanism in order not to over-produce very massive galaxies in the largest virialized DM halos at low redshift (Croton et al., 2006).

It is not clear, however, how such a feedback mode can effectively couple the SMBH mass with the structural properties of their galactic hosts and give rise to the observed scaling relations. For such a task, modelers have instead turned to feedback modes associated to the phases of fast SMBH growth in bright QSO.

In all feedback models in which the black hole energy injection is very fast (explosive), if strong QSO feedback is responsible for rapidly terminating star formation throughout the entire bulge (Di Matteo et al., 2005), QSOs and, in general, type-1 AGN are associated with the final stage of bulge formation. Then, very little evolution, as well as very little scatter, is expected for the scaling relations, and it is very hard to produce any positive offset like the one observed.

The physics of such ("quasar") mode of AGN feedback remain elusive, as it remains the issue of whether the energy release by the associated process of rapid black hole growth is indeed 
responsible for halting the conversion of gas into stars on galactic (kpc) scales, or whether it is only responsible for a milder form of "self-regulation" by cutting off its own gas supply on nuclear (pc) scales (Hopkins et al., 2009).

\section{Cosmology II: AGN feedback}

The phenomenological investigation presented in $\S$ above leaves open the fundamental question about the physical origin of such a clear, parallel differential growth of both the black holes and the galaxy population.

From the discussion of AGN activity in Chapter "Active Galactic Nuclei" by E. Perlman in this same volume, it should be immediately apparent that black hole growth is often, if not always, accompanied by the release of enormous amounts of energy, in the form of radiation, outflows, and gravitational waves.

Black holes accreting at high rates in the so-called radiative (or quasar) mode will release of order $10 \%$ of the accreted rest mass energy as radiation. They can also drive broad (uncollimated) outflows, again described in more detail in chapter "Active Galactic Nuclei" by E. Perlman in this book, and in about $10 \%$ of bright AGN, radio emitting, relativistic jets are observed (the quasar 3C273 seems to be such an object that is accreting efficiently and making powerful jets at the same time).

But even black holes in the so-called inefficient accretion regime, where cooling is dominated by advective processes rather than radiation, can drive powerful, collimated outflows in the form of relativistic jets. Perhaps the best example of such a powerful "low efficiency" black hole is the radio galaxy Virgo A, which is the product of inefficient accretion onto the supermassive black hole at the center of M87, which itself is located in the center of the Virgo cluster. See $\S$ "Active Galactic Nuclei" by E. Perlman in this book for a detailed discussion of the properties of the M87 jet.

This energy will be released directly into the environment from which the black hole grows: the cooling, possibly star-forming gas in the central galaxy. Any transfer of energy to the gas should thus reduce the rate at which gas cools and forms stars. While the direct link between star formation through cooling in the centers of galaxies and black hole growth through accretion is not fully established, it is easy to imagine how such an energy deposition can reduce the rate of accretion onto the central black hole as well.

This process of cooling-induced black hole activity can therefore be considered as a negative feedback loop, in that increased accretion activity acts to decrease the large scale gas supply to the black hole. The impact on star formation might be coincidental (if black hole growth is unrelated to the actual star formation rate) or fundamental (if black hole growth is mediated or directly fueled by star formation, for example, through direct accretion of stars).

Furthermore, while the direct link between black hole growth and star formation suggested by the $M-\sigma_{*}$ relation is hidden, and evidence for the suggested underlying feedback process on stars is largely circumstantial (as will be discussed below), some important clues can be derived by tracing the evolution of the feedback energy released by growing black holes as a function of black hole mass and redshift.

In this section, we describe in some detail how such an inventory can be made and how feedback itself operates. We will focus primarily on the information about the properties of 
black holes that can be extracted from observations of feedback, with other chapters discussing the role of black hole growth on the formation of structure in more detail (see $\S$ "Clusters of Galaxies" by R. Bower in this book).

\subsection{Evidence and arguments for feedback}

The process of accretion is a multi-scale phenomenon: The range from the place of capture, where the gas first enters the sphere of influence of the black hole, to the event horizon of the black hole spans roughly seven orders of magnitude in scale - too much to simulate in one big simulation for even just one dynamical time on the outer scale.

Yet, as extreme as this range in scales may be, the process of feedback can cover another 5 orders of magnitude more in scale: From the scales of the horizon (about AU-size for a typical central black hole in a typical, $L_{*}$, galaxy) to scales of entire galaxy clusters (several hundred kiloparsec): a dynamic range of twelve orders of magnitude.

Given that our understanding of accretion is still developing, and that our understanding of jet formation is, at best, elementary, it should not come as much of a surprise that our understanding of AGN feedback is mostly limited to fairly crude statements about energy input and global heating efficiencies from a theoretical perspective.

The best observational evidence for feedback is not on galaxy scales at all, but on the largest spatial scales on which we can expect black holes to have any meaningful influence: In the centers of galaxy clusters. The reason for this is twofold:

- First, the angular scales on which feedback in galaxy clusters unfolds are readily resolvable by telescopes in all bands of the electromagnetic spectrum.

- Second, the signatures of AGN feedback in galaxy clusters are easy to identify from X-ray and radio imaging, as we will discuss momentarily.

Consequently, we have developed a fairly mature picture of how AGN feedback works on the very largest scales and have even successfully simulated the feedback processes in computers.

On smaller scales, the evidence for feedback becomes increasingly circumstantial. Thus, while the link between black hole and galaxy properties may be the most fundamental expression of direct coupling of their growth processes, it is also the most elusive in terms of direct evidence for this coupling.

A number of reasons conspire to limit our observational insight into galactic scale feedback:

- The angular scales of this process are inherently small, given that the feedback must be happening in the centers of galaxies.

- While cluster evolution is happening in the current epoch, at low redshift, galaxy growth happens at higher redshift - during the star formation epoch. This is especially true for the galaxies that seem to require AGN feedback the most.

- Both star formation and rapid black hole growth tend to cloak themselves in dust extinction and photo-electric absorption. It may be that the smoking guns of feedback are mostly hidden behind Compton-thick X-ray absorbers and many magnitudes of dust extinction. 
Finally, the tight connection between black hole growth and star formation suggested by the $M-\sigma_{*}$ relation and by the similarity in the redshift evolution of both populations seems to imply that stars and black holes grow roughly simultaneously. For feedback to have a strong impact on star formation and at the same time couple the mass of growing black holes to the mass of stars in a galaxy, one would expect rapid black hole growth to be concurrent with episodes of star formation.

This would imply that feedback on star formation would have occurred during the quasar phases, and since most quasars are radio quiet, this suggests that at least part of the feedback on star formation operates through a different channel than the readily observable "radio galaxy" feedback on cluster scales at low redshift. Given that this feedback must have occurred during the quasar epoch, it is commonly referred to as "quasar mode" feedback.

In fact, the most convincing "evidence" for such a mode comes not from actual observations of black holes but from semi-analytic models of galaxy formation: In order to explain the galaxy luminosity function and galaxy color distribution, modelers have to assume two types of feedback: One that disperses and heats the star-forming gas at the end of a star-formation cycle (generally triggered by mergers), effectively halting star formation — this is the "quasar mode" — and one that maintains the gas in typical elliptical galaxies in its tenuous, hot state - this is the "radio" or "maintenance mode" (Springel et al., 2005; Croton et al., 2006).

However, these models say nothing about the actual physical mechanism of feedback: they assume quasi-spherical heating of the gas in both "quasar" and "radio" mode, and the only thing that distinguishes them is the prescription of how the black hole accretes (whether from cold or hot gas). The more appropriate naming convention is thus "cold" and "hot" mode accretion.

Thus, the circumstantial evidence for "cold" mode feedback does not answer the question of whether the heating/dispersal occurs as a result of winds, jets, or radiation released by the accreting black hole. Given that slowly growing black holes are radiatively inefficient and universally seem to be radio-active ( $\mathrm{Ho}, 2008)$, it has generally been assumed that any feedback from black holes in the "hot" mode must be in the form of jets.

\subsection{Feedback in galaxy clusters}

It is instructive to begin by discussing the obvious examples of AGN feedback. This will inform our discussion of the possible influence of AGN on the process of star formation on galactic scales. In particular, from a discussion of radio galaxy feedback on cluster scales it is possible to draw quantitative conclusions about "radio-mode" or "hot-mode" feedback by jets from slowly growing black holes. For a more detailed discussion of feedback in galaxy clusters, see McNamara \& Nulsen (2007).

On a basic level, the importance of feedback was already apparent with the discovery of powerful radio galaxies in the 1960s and onward (though the relevance of the black hole in this context took longer to establish): Radio galaxies, like the example of Cygnus A shown in Fig. 25, exhibit diffuse "lobes" of synchrotron emission (see chapter "Active Galactic Nuclei" by E. Perlman in this same volume), on scales of tens and even hundreds of Kiloparsec. In other words: The action of a jet from the central black hole deposits magnetized relativistic plasma into the surrounding medium.

Simply summing up the entire synchrotron radiation and making reasonable assumptions about the shape and volume filling fraction of the emitting regions, it is straight forward to 

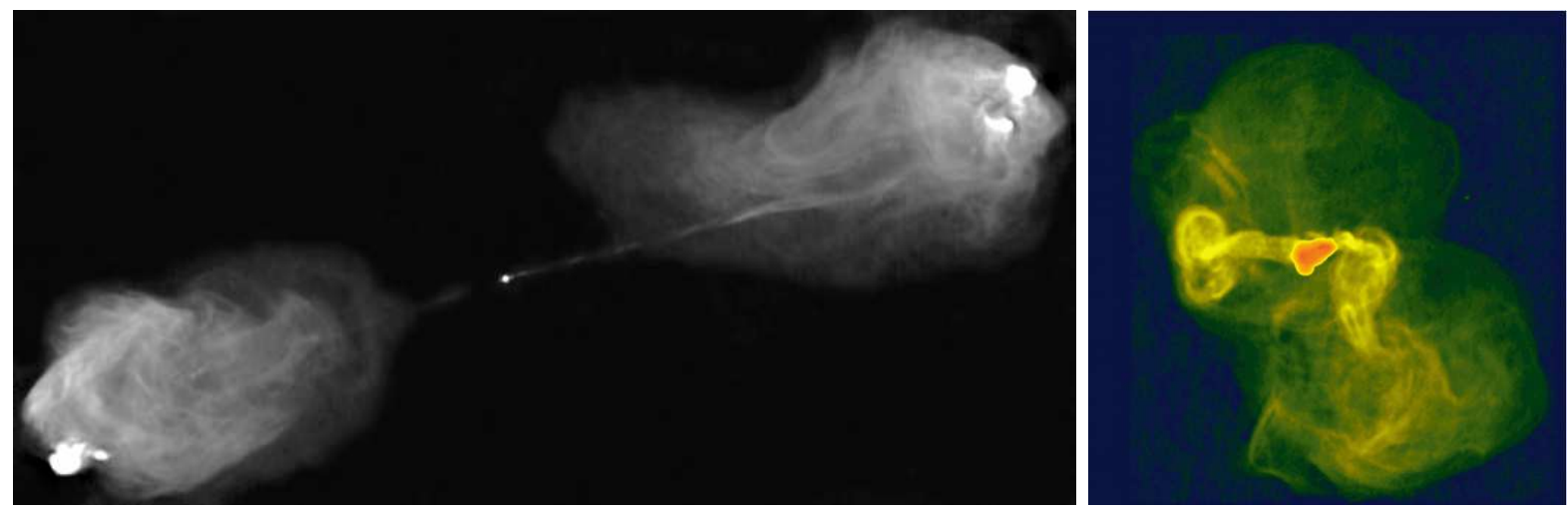

Figure 25: Left: The FRII radio galaxy Cygnus A, observed by the VLA at 6cm; image scale: $150 \mathrm{kpc} \times 85 \mathrm{kpc}$; right: The large scale structure of the FR I radio galaxy Virgo A, observed by the VLA at $90 \mathrm{~cm}$. The relativistic inner jet of M87 is contained in the over-exposed central radio lobes; image scale: $80 \mathrm{kpc} \times 80 \mathrm{kpc}$.

derive lower limits on the total energy needed to explain the radio emission. In some cases, the minimum energy derived could be enormous: Perley et al. (1984) found that Cygnus A required at least $E_{\min } \gtrsim 10^{60} \mathrm{ergs}$ pumped into the lobes by the central black hole. For perspective: This is of the same order as the gravitational binding energy of the Milky way.

If jets could release this much energy in relativistic gas into the environments of black holes, it would be hard to imagine how the environment could not be strongly affected.

The first direct evidence for feedback on the gas surrounding the black hole came with the arrival of high-resolution X-ray imaging: using ROSAT data, Boehringer et al. (1993) discovered that the radio galaxy Perseus A (powered by the supermassive black hole in the central cluster galaxy NGC 1275) excavates large cavities in the hot, X-ray emitting thermal gas that fills the Perseus cluster. The gas is pushed aside into dense shells and the excavated X-ray cavities are filled with radio emission by the lobes of the radio galaxy (see Fig. 26)

Similarly, the privileged view we enjoy of the nearby radio galaxy Virgo A (also known as M87) allowed a uniquely detailed study of its multi-scale emission well before the idea of feedback had taken hold. The inner (roughly kpc) jet of M87 is discussed in some detail in chapter "Active Galactic Nuclei" by E. Perlman in the same volume. However, lower frequency observations revealed a much richer picture on scales just outside of the visible galaxy, still in the very center of the Virgo cluster (Owen et al., 2000): Curling and twisting strands of radio emission, connecting the nucleus to a set of radio lobes about $20 \mathrm{kpc}$ in radius, and misaligned with respect to the central jets by about 90 degrees on the sky 6 .

Both Perseus and Virgo are cool core clusters, and in particular Perseus had long been considered a prototypical example of a cooling flow. That is, the radiative cooling time in the center of the cluster is shorter than the age of the cluster. In a quasi-hydrostatic model of a cluster (inward gravity, mostly provided by the dark matter contribution, balanced by an

\footnotetext{
${ }^{6}$ Part of this misalignment could be due to projection, of course, given that at least the inner jet is directed fairly close to the line-of-sight.
} 
outward thermal pressure gradient), this would imply that the cluster must be contracting on a Kelvin-Helmholtz timescale, with gas at the center cooling rapidly to star-forming temperatures. Cooling gas from further out in the cluster would replace the gas in a slow, sub-sonic inflow (for a review, see Fabian, 1994, and references therein).

Even before the era of Chandra and XMM it was already apparent that this simple picture of ongoing inflow of cooling gas did not accurately describe cool core clusters: The implied rates for star formation were an order of magnitude higher than the observed rates.

Radio surveys of cluster centers revealed that essentially all traditional "cooling flow" clusters had active radio galaxies in their centers (Burns, 1990). Generally, these radio sources are Fanaroff-Riley type I sources (henceforth abbreviated as FR I; see chapter "Active galactic nuclei" by E. Perlman in this book for a discussion of radio source morphology), though it is not entirely clear whether this is due to lower average source power compared to field FR II galaxies or due to the increased gas density in clusters (frustrating source evolution and possibly leading to increased entrainment).

Guided by the detailed examples of feedback in the Virgo and Perseus clusters and the observed mismatch between the X-ray cooling rate and the star formation rates in clusters, the first models of black hole feedback in the context of galaxy clusters were presented in Tabor \& Binney (1993).

\subsubsection{The Chandra and XMM-Newton view}

The role of AGN in regulating the cooling of gas in cool core clusters was brought into clear focus with the launches of Chandra and XMM-Newton in two ways:

Chandra observations revealed the presence of cavities just like those found in the center of Perseus in virtually every cool core cluster, providing the observational confirmation that the radio galaxies present in these clusters actively perturb the gas. Generically, the cavities appear to be surrounded by relatively cool ga:77. Deep Chandra observations sometimes reveal multiple cavities on different scales, which has been interpreted as evidence for variability in the AGN powers.

At the same time, XMM-Newton's high-resolution X-ray spectra of the cluster centers revealed that radiative cooling must be impeded below a threshold temperature of order 10 million degrees (about $1 \mathrm{keV}$ ), at a temperature where cooling should be efficient and rapid due to the flattening of the cooling curve for thermal gas as atomic line cooling becomes dominant (Peterson et al., 2003).

This result is consistent with the observed lack of star formation in central cluster galaxies, compared to the hundreds of solar masses of star formation per year that would have been expected based on simple cooling flow models. It moves the discrepancy of cool gas missing at molecular temperatures to cool gas missing at X-ray temperatures below about a keV. In either case, a heating agent is needed, but in the "revised" cooling flow problem, the gas that must be preferentially targeted is at about a $\mathrm{keV}$ and will thus occupy a much larger volume fraction, which should make it easier to interact with for any feedback mechanism.

\footnotetext{
${ }^{7}$ This was surprising because one might naively expect the gas most strongly affected by feedback to be hot.

${ }^{8}$ But see Morsony et al. (2010) for arguments why the presence of cavities is not a sufficient argument for AGN duty cycles.
} 

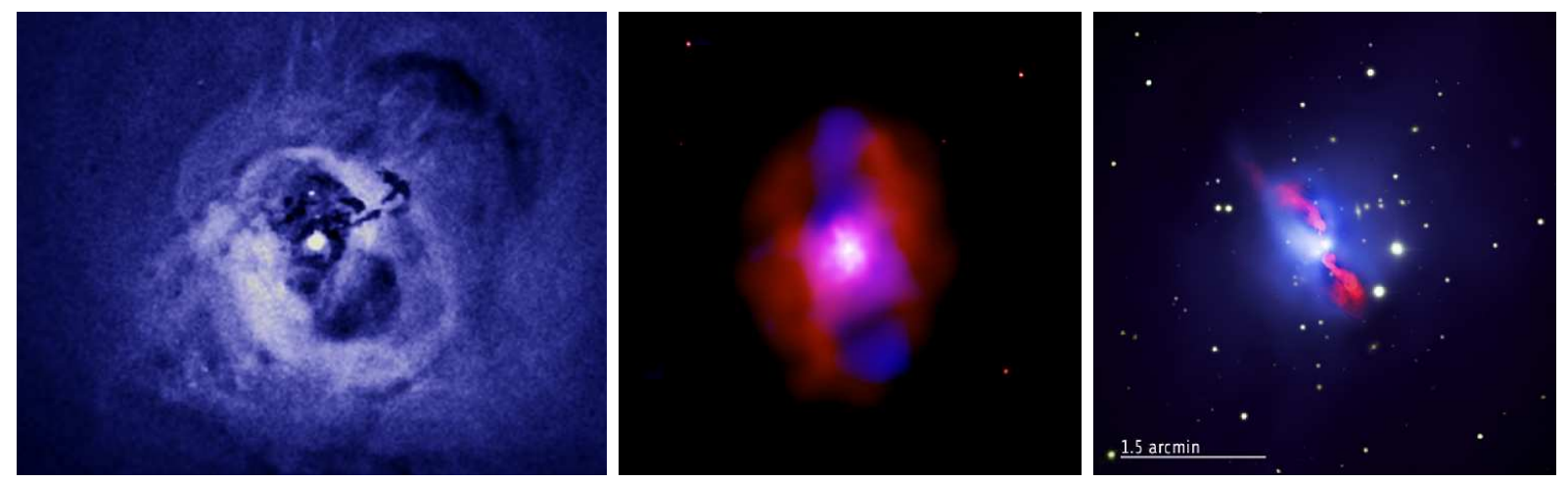

Figure 26: Left: Deep Chandra observation of the Perseus cluster (Fabian et al., 2006); middle: Chandra image of MS0735 (red) and VLA (blue), adopted from McNamara et al. (2005); right: Chandra image of Hydra A (blue) and $6 \mathrm{~cm}$ VLA radio image (red), adopted from NASA (Kirkpatrick et al., 2009); image scale: $80 \mathrm{kpc} \times 80 \mathrm{kpc}$.

Thus, after about a decade of study, a standard paradigm has emerged from the high incidence of radio loud AGN in cool core clusters and from the theoretical requirement of a heating agent that maintains the temperature distributions in galaxy clusters: Radio galaxies provide the energy needed to counterbalance cooling in the centers of clusters.

From these observations, and from theoretical modeling (Begelman \& Cioffi, 1989; Reynolds et al., 2001), a simple understanding of radio source evolution has been developed that underpins the radio galaxy feedback paradigm. According to this picture, radio source evolution separates into three stages (e.g. Reynolds et al., 2001):

1. In the initial supersonic phase, jet plasma inflates cocoons that are strongly over-pressured relative to the environment. These cocoons must expand, and the rapid energy release implies that this expansion is supersonic in the frame of the environment.

The expansion is similar to that of a wind-blown bubble described by Castor et al. (1975): The cavity radius roughly follows the self-similar scaling 9

$$
R \propto\left(\frac{P_{\text {jet }} t^{3}}{\rho_{\mathrm{ICM}}}\right)^{1 / 5}
$$

which is functionally equivalent to the Sedov-Taylor solution if the blast energy is replaced by the injected energy over time, $P_{\text {jet }} t$. In this expression, $\rho_{\mathrm{ICM}}$ is the density of the environment.

Note that this argument neglects the actual jet propagation completely and assumes the jet energy is randomized as the jet encounters the environment (in more powerful FR II sources) or through entrainment (which has been suggested as the dissipation agent in FR I source; Laing \& Bridle 2002).

\footnotetext{
${ }^{9}$ This expression applies to bubbles smaller than a cluster pressure scale height. It is straight forward to extend it to stratified powerlaw atmospheres.
} 
2. As the source expands, the pressure inside the cocoon and in the shell eventually approaches the pressure of the environment and the expansion becomes sub-sonic. The initially generated shock wave of the supersonic expansion will continue to coast outward, leaving behind a sub-sonically expanding cavity. As the expansion velocity becomes subsonic, the confinement is dominated by the thermal pressure of the environment, so the solution changes to a pressure confined bubble,

$$
R \propto\left(\frac{P_{\text {jet }} t}{p_{\mathrm{e}}}\right)^{1 / 3}
$$

3. Finally, as the source expansion velocity drops below the buoyancy speed or once the pressure of the source drops below the dynamic pressure of motions in the environment, the cavities/cocoon will detach and float away from the black hole buoyantly or advectively.

Once cold gas has refilled the central region of the cluster, any jet activity will start the cycle anew.

It is clear from eq. (15) that jet power and density are the controlling variables in the initial evolution: Lower power jets in denser environments will be more easily frustrated (with more slowly expanding cocoons that become sub-sonic and unstable at smaller sizes).

\subsubsection{Estimating the kinetic power of a radio source}

Before the discovery of X-ray cavities, measurements of the kinetic power of radio galaxies (that is, the total power traveling down the jets) were limited to estimates based on the observed synchrotron emission (see discussion in 4.2 ). These were hampered by several factors:

- Without knowledge of the field strength, an observed synchrotron flux, along with an estimate of the emitting volume could only provide a lower limit of the total energy in the radio plasma (essentially assuming equipartition between the energy in electrons and magnetic field).

- The estimate of the volume depends strongly on the volume filling fraction, which is not measurable.

- Synchrotron aging can cause electrons to cool and develop a sharp cutoff in the synchrotron spectrum. Thus, significant amounts of energy in lower-energy electrons would be unaccounted for in the total power budget.

One of the most important results from the discovery of X-ray cavities in clusters is a robust, independent way to estimate the power of cluster radio sources. It is based on the fluid mechanics of inflated bubbles: In order to inflate a cavity in the intra-cluster gas, the jet must (a) displace the material in the environment into a shell surrounding the cavity, which is of the order of $E_{p V} \sim p V$ (and depends on the details of the inflation history of the bubble) and (b) replace it with relativistic, magnetized gas. At a minimum, the amount of energy needed to do this is the work done on the cluster gas and the internal energy of the radio plasma. If the expansion of the cavity is adiabatic, the total energy needed is

$$
E_{\text {cavity }}=\frac{\gamma p V}{(\gamma-1)} \sim 4 p V
$$


where $\gamma$ is the adiabatic index of the gas inside the cavity and is typically assumed to be $\gamma=4 / 3$, given the presence of relativistic electrons and tangled magnetic fields.

Estimating the age of the cavity, and thus the jet power needed to inflate it, is more difficult and introduces some uncertainty. Direct kinematic measurements of the expansion velocities of cavities are impossible with current X-ray telescopes. However, in many cases, the observations suggest that the temperature of the shells surrounding the cavities is low. In this case, it is safe to assume that the recent expansion of the cavity was sub-sonic. Thus, the sound crossing time of the bubble radius is a reasonable lower limit on the cavity age:

$$
t_{\text {cavity }} \geq \tau_{\text {sonic }}=\frac{R_{\text {cavity }}}{c_{\mathrm{s}}}
$$

where $R_{\text {cavity }}$ is the radius of the cavity and $c_{\mathrm{s}}$ the sound speed of the cluster gas.

Given that most cavities are found in cluster centers, a reasonable upper limit on the age of the cavity is the buoyant rise time $\tau_{\text {buoy }}$ of the bubble in the gravitational potential of the cluster (since an bubble older than $\tau_{\text {buoy }}$ would have risen out of the cluster center):

$$
t_{\text {cavity }} \leq \tau_{\text {buoy }} \sim \frac{2 R_{\text {cavity }}}{v_{\text {buoy }}} \sim \frac{2 R_{\text {cavity }}}{c_{\mathrm{s}} \sqrt{\frac{4}{3} \frac{d \ln (P)}{d \ln R} \frac{1}{C_{\mathrm{W}}}}}=2 \tau_{\text {sonic }} \sqrt{\frac{3 C_{\mathrm{W}}}{4} \frac{d \ln (R)}{d \ln (P)}} \sim 2 \tau_{\text {sonic }}
$$

where $C_{\mathrm{W}}$ is the drag coefficient of the rising cavity and typically assumed to be of order $C_{\mathrm{W}} \sim 0.5$ and the exact numerical value of the expression under the square root depends on the pressure scale height of the cluster in bubble radii, but should be of order unity for typical observed bubble radi10. For detached cavities, the appropriate age to use is the buoyancy time for the projected distance instead of the bubble diameter.

Given that buoyancy sets in after the source becomes sub-sonic, this loosely brackets the power inferred for the jets from the measurement of cluster cavities to

$$
\frac{E_{\text {cavity }}}{\tau_{\text {buoy }}+\tau_{\text {sonic }}} \sim \frac{E_{\text {cavity }}}{3 \tau_{\text {sonic }}} \lesssim P_{\text {jet }} \lesssim \frac{E_{\text {cavity }}}{\tau_{\text {sonic }}}
$$

Estimates of the central cluster density and temperature (and thus pressure and sound speed) are readily obtained from X-ray images and spectra. The most difficult part is the estimate of the cavity volume, since errors in the estimated cavity radius and viewing angle uncertainties can compound to errors of up to an order of magnitude in source power. Nonetheless, this method has afforded us with a large number of reliable estimates of jet powers to within a factor of a few for dozens of radio galaxies in nearby clusters11.

While early, shallower Chandra exposures of cluster centers only showed cool shells in the vicinity of the cavities, deep observations of a number of important clusters later also showed the presence of shocks surrounding at least some of the cavities (McNamara et al., 2005; ; Wise et al., 2007; Forman et al., 2007, e.g.). Given the generic picture of how radio sources evolve over time described in 4.2.1, the presence of weak shocks should be expected (and had been predicted in Reynolds et al. 2001).

\footnotetext{
${ }^{10}$ The buoyancy speed can never exceed the sound speed.

${ }^{11}$ It should be kept in mind that the inferred powers are averages over the cavity age, which can be between millions to hundreds of millions of years old.
} 
In fact, an important corollary from eq. (15) is that the initial strongly supersonic phase is short lived in typical cluster environments: The expansion velocity of the shell is

$$
v_{\text {shell }}=\frac{d R}{d t} \propto\left(\frac{P_{\text {jet }}}{\rho_{\mathrm{ICM}} R^{2}}\right)^{1 / 3} \propto R^{-2 / 3} \propto t^{-2 / 5}
$$

Given that cavities should remain in the cluster centers for about 2-3 sound crossing times before becoming buoyant, the fraction $f_{>M}$ of time a given radio galaxy spends expanding super-sonically at or above a given Mach number $M$, relative to the total dynamic lifetime before buoyant removal, should only be of order

$$
f_{>M} \sim \frac{\tau_{>M}}{3 \tau_{\text {sonic }}} \sim \frac{1}{3} M^{-5 / 2}
$$

Thus, the observational lack of evidence for sources expanding at large Mach numbers does not rule out that radio sources go through this strong shock phase. It is, however, short lived and only a small mass fraction of AGN's environment passes through a strong shock.

The detection of a shock (which requires not just the detection of a surface brightness jump, but also a temperature jump, which is most easily identified through a harder X-ray color) offers a significantly better diagnostic of jet power than the cavity method. Because the shock strength is an indication of the expansion velocity, a measured shock radius, brightness, and strength can be modeled using simple 1D spherical shock models to give a reliable source power. Typical Mach numbers for shocks detected in clusters are between one and two, consistent with this argument.

This rather simple parametric description of radio source evolution is complemented by a growing body of numerical simulations of jet-driven feedback. Initial 2-dimensional simulations generally supported the simple picture (Reynolds et al., 2001). However, a thorough understanding requires full 3-dimensional simulations, and work on understanding the details of feedback $a b$ initio is still in the early stages.

A key problem posed by $2 \mathrm{D}$ and early $3 \mathrm{D}$ simulations is the apparent contradiction of highly bipolar release of energy in the jet and the need for mostly isotropic heating (Vernaleo \& Revnolds, 2006). While outgoing shocks assume a spheroidal shape relatively quickly, heating by shocks is insufficient to solve the cooling flow problem (see eq. 22). In addition, jets evacuate cocoons around them. Ongoing, unidirectional jet activity was found to propagate inside this cocoon, dynamically and energetically isolated from the inner cluster gas and thus unable to counteract cooling in the center.

The solution might lie in the interaction with the cluster and in the internal dynamics of jets: Heinz et al. (2006) found that the jets can continue to efficiently couple to the inner cluster if their axes are subjected to a moderate wobble and, crucially, the cluster itself is dynamically evolved in the context of a cosmological simulation. Simulations generally show that the complex X-ray appearance of clusters, and even the appearance of multiple successive cavities, can be generated by a single, ongoing episode of jet activity and a dynamic cluster atmosphere (Morsony et al., 2010). This suggests that the interaction between jets and clusters goes both ways: cluster dynamics affects jet dynamics and vice versa. 

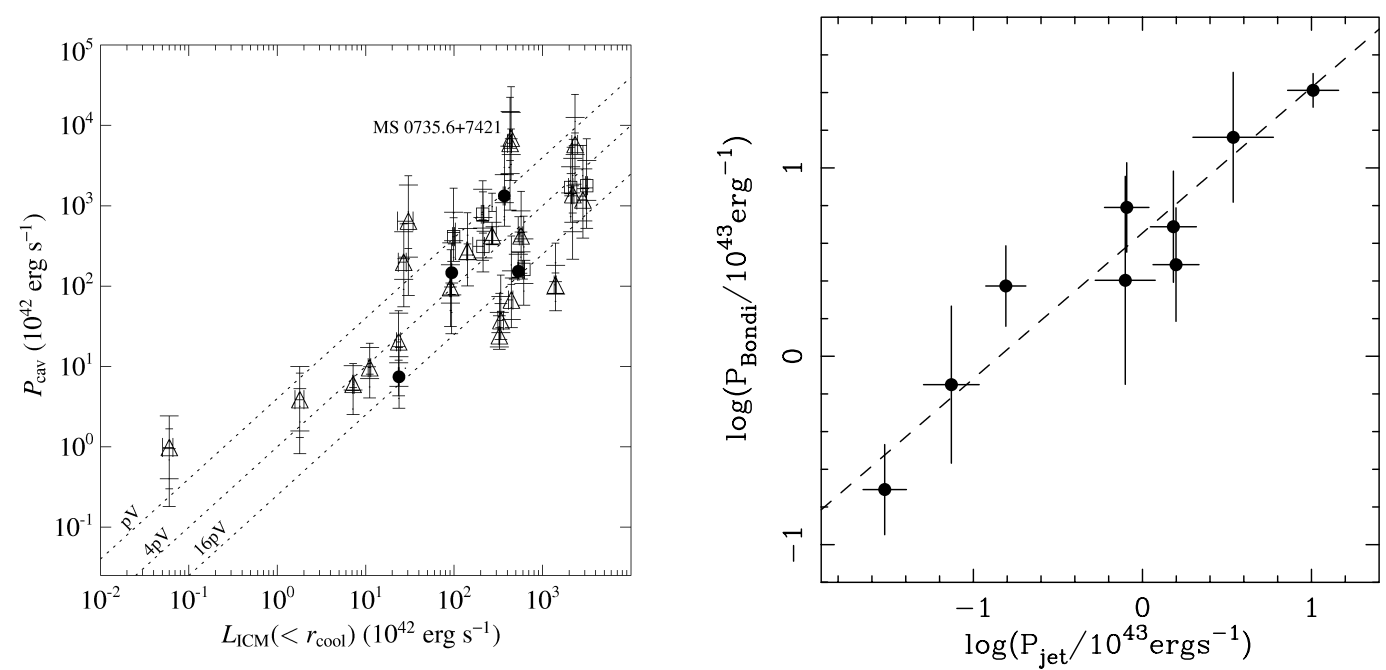

Figure 27: Left: jet power inferred from cluster cavities plotted against X-ray cooling luminosity from within the cluster cooling radius, adopted from Rafferty et al. (2006); the diagonal lines indicate the efficiency required for the jet power to offset cooling, with the line labeled as $4 p V$ equivalent to $100 \%$ efficiency, adopted from Rafferty et al. (2006); right: jet power plotted against Bondi accretion rate, adopted from Allen et al. (2006).

\subsubsection{Cluster radio sources as a population}

A census of nearby clusters with cavities reveals a number of important insights about the statistical and global properties of central cluster radio sources:

- When plotting jet power vs. X-ray luminosity, radio sources straddle the heating=cooling line: About half of the radio sources sampled have kinetic powers that are higher than the cooling luminosity of the cluster (see Fig. 27 adapted from Rafferty et al. 2006).

- More importantly, the jet power appears to be correlated with the cooling luminosity of the cluster: More powerful radio sources are found in clusters with higher cooling rates. This is exactly the signature one would expect in an AGN feedback picture (Rafferty et al., 2006).

- The jet power measured from cavities appears to be related to the Bondi accretion rate of the cluster. That is, in clusters with higher central densities and/or lower core temperatures, the jet power is higher (Allen et al., 2006). While this correlation does not imply that black holes actually accrete at the Bondi rate in clusters, the relatively close match suggests that black holes accrete directly from the cluster gas as it flows into the central galaxy (see Fig. 27).

Together, these results suggest that the balance between heating and cooling in clusters is relatively tight. In fact, the high efficiencies suggested by these findings, in a global sense, as well as from studies of individual, powerful black holes with relatively low masses, have led to 
the suggestion that the black holes powering the jets in cluster centers might even require the extraction of black hole spin (McNamara et al., 2011).

However, given the significant uncertainties in the jet power estimates, and given that clusters are, in all likelihood, not steady state systems (thus, cooling rates can temporarily exceed heating rates, as long as they are balanced on average), a sufficient region of parameter space is still allowed in which AGN can balance cooling without requiring extreme efficiencies, accretion rates, or black hole masses.

All of the detailed studies of radio galaxy feedback in clusters have been limited to clusters at relatively low redshifts, because of the need for high-fidelity X-ray images of cavities and shocks. In addition, high-redshift cluster samples are sparse. Consequently, constraints on cluster feedback at higher redshift are much harder to obtain. Radio surveys of high-redshift clusters do indicate an increase in the cluster radio luminosity function (Branchesi et al., 2006) hinting at an increase in feedback activity.

Finally, it is worth mentioning that, while the central massive elliptical galaxies harbor by far the most massive black holes in clusters, other cluster galaxies can be radio loud. This situation typically leads to the formation of a bent radio source. The exploration of the statistics of non-central cluster radio sources is ongoing, and it has been suggested that this population of sources could contribute to cluster feedback (Hart et al., 2009). However, it is difficult to envision a scenario whereby a population of such sources will dominate the heating rate in the average cluster. It is also unclear how they could respond to central gas cooling as would be required for thermal regulation of the cluster gas.

\subsection{Feedback in groups}

Due to the lower temperatures and densities in the intra-group medium, detecting evidence for group-wide feedback from X-ray observations is significantly more difficult than in clusters.

Statistical X-ray studies of cluster and group samples show that, generically, lower mass halos have excess central entropy when compared to self-similar models of halo formation, indicating that an additional source of non-gravitational heating must have injected entropy preferentially

into the low mass systems (e.g. Ponman et al., 2003). AGN have been suggested as a possible heating mechanism (e.g. Short \& Thomas, 2009). Figure 28 shows how the inclusion of feedback in detailed models of cluster and group atmospheres affects primarily low mass systems and raises their central entropies.

And, in fact, surveys of groups suggest that such a scenario might work: Dong et al. (2010) find that a fraction of at least $25 \%$ of the groups in their survey contain clearly detectable cavities, with a clear preference for cavities to be found in groups with cooler cores (as is the case in clusters). In their sample, the presence of cavities does not appear to be correlated with the $1.4 \mathrm{GHz}$ radio flux.

On the other hand, a survey of radio properties of groups shows that the central temperatures of groups with central radio sources are elevated compared to a radio quiet sample of groups, which was suggested as a possible indication of ongoing radio galaxy feedback (Croston et al., 2005). 

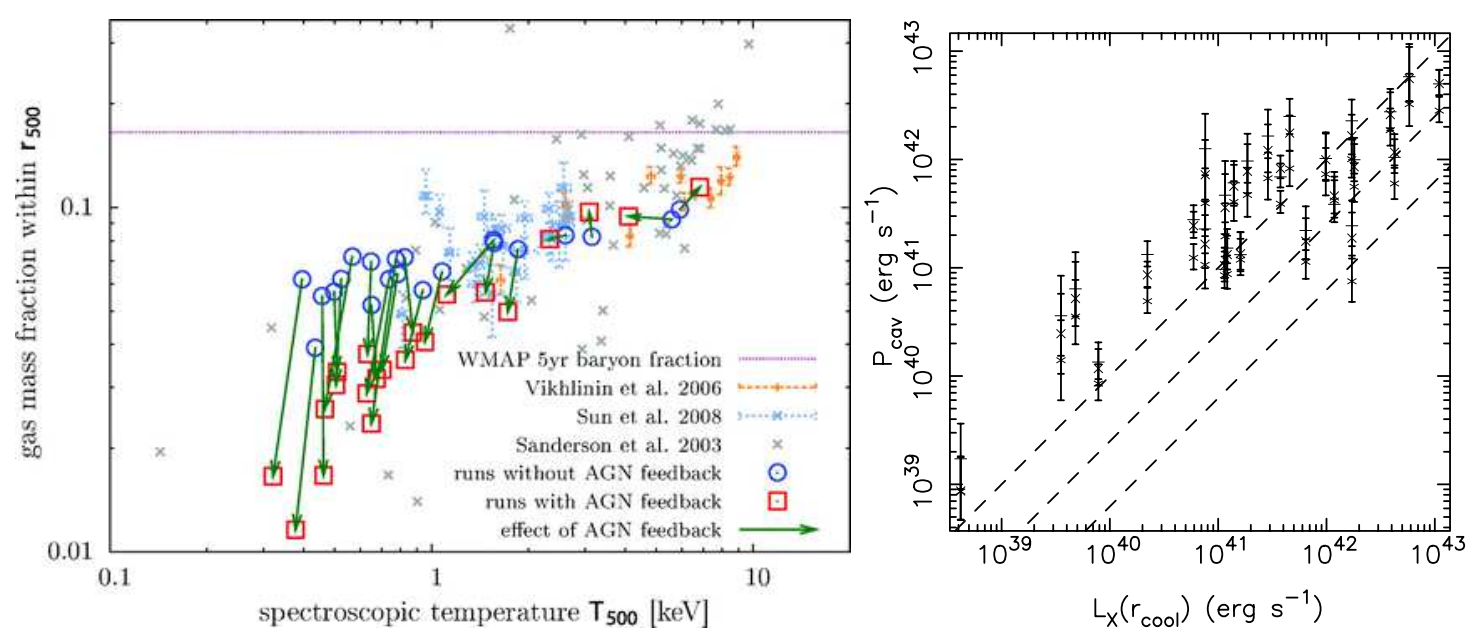

Figure 28: Left: Simulations show the increasingly important effect of AGN feedback on lower mass clusters and groups; plotted is the central gas mass fraction as a function of virial temperature (mass); adopted from Puchwein et al. (2008) ; right: same plot as in the left panel of Fig. 27, but for atmospheres of elliptical galaxies instead of clusters; adopted from Nulsen et al. (2007).

\subsection{Radio mode feedback in galaxies}

The best direct evidence for black holes affecting the surrounding gas within galaxies comes again from combined radio and Chandra observations of nearby objects: Fig. 29 shows images of three nearby galaxies where jets clearly excavate cavities and affect gas on sub-galactic scales: M87, M84, and NGC5128 (Centaurus A). In the latter case, the entrainment of gas into the jet, and the formation of a strong shock driven by the expanding south-western radio lobe is directly visible in X-rays.

These are clear local examples of relatively powerful, evolved radio galaxies. Numerous other examples have been studied individually. Yet, perhaps the most convincing argument for the importance and prevalence of jets in massive galaxies come from statistical studies.

In a study of X-ray cavities in and around nearby ellipticals, Nulsen et al. (2007) found that AGN power more than matched the cooling luminosity of the gas in the hot galactic X-ray halo; compared to the same study in clusters (shown in the left hand panel of Fig. 27), the galaxies fall consistently above the heating=cooling line (McNamara \& Nulsen, 2007).

The implication that radio-mode feedback is important in massive galaxies (those that have detectable X-ray halos) is complemented by statistical studies of the radio source incidence in galaxies of different type and mass: The left panel of Fig. 30 shows a steady increase of the fraction of radio loud galaxies (that is, galaxies above a fixed radio luminosity per stellar mass) with stellar mass of the host galaxy (Best et al., 2005). The most massive galaxies exhibit the highest radio fluxes per stellar mass, indicating that radio mode feedback is most active in today's most massive galaxies, with $10 \%$ or more of the most massive galaxies hosting radio loud AGN.

Given that more massive galaxies harbor more massive black holes, one might naively expect 

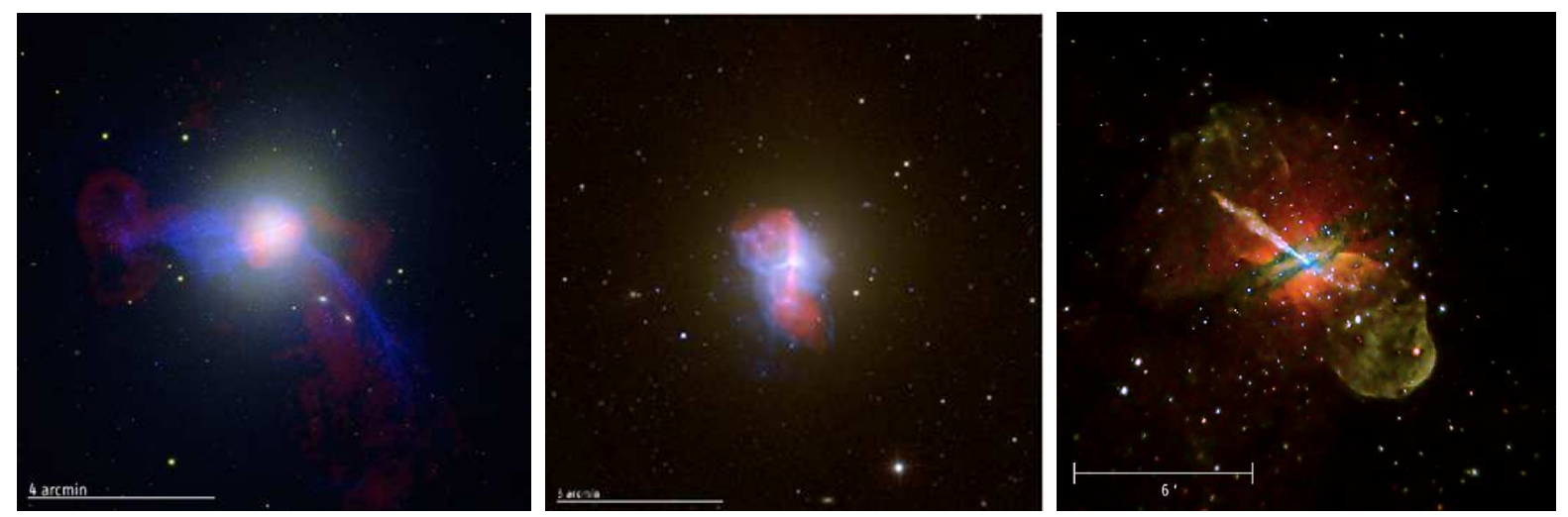

Figure 29: Left: multi-wavelength image of the central region of the Virgo cluster, showing the influence of the black hole on sub-galactic scales; middle: multi-wavelength image of the galaxy M84 in the Virgo cluster (blue: radio, red: X-ray, yellow: visible); right: multi-wavelength image of Centaurus A.

this result to suggest that radio loudness is a fraction of black hole mass. Early studies seemed to suggest this (e.g. Franceschini et al., 1998). However, deep radio surveys of a wider class of black holes show that the radio loudness, defined relative to the bolometric flux, actually increases for decreasing Eddington ratios (Ho, 2002): The right panel of Fig. 30 shows that radio emission increases in relative brightness (compared to the bolometric luminosity) for lower luminosity AGN.

This suggests that black holes become relatively more efficient at liberating energy in the form of jets as their luminosity (and presumably accretion rate) drops, such that all black holes at sufficiently low accretion rate appear to be driving some form of radio-loud outflow.

The idea that low luminosity black holes are universally radio loud arose roughly in parallel also in the study of X-ray binary black holes (Gallo et al., 2003), where it is possible to track individual black holes across outburst and decline into quiescence. These observations showed that a jet was always present at low luminosities, with increasing relative radio flux at decreasing X-ray luminosities.

While a thorough theoretical understanding is still missing, it has been shown that lowefficiency accretion leads to the formation of geometrically thick flows, as opposed to the geometrically thin accretion disks found in, for example, Seyfert galaxies and quasars. In such a geometrically thick (quasi-spherical) flow, it might be much easier to build up significant magnetic flux, even just from stochastic turbulent dynamo processes in the disk, which could in turn drive the jet (see chapter "Active Galactic Nuclei" by E. Perlman in this book).

Anecdotally, the case of the M87 jet makes the perfect illustration of this point: The total radiative output from the black hole is unimpressive 12 at $L_{\mathrm{M} 87} \sim 10^{41} \mathrm{ergs} \mathrm{s}^{-1}$, about two orders of magnitude below the luminosity corresponding to efficient accretion at the Bondi rate of that particular black hole. Meanwhile, the estimated jet power from this object is about two orders of magnitude larger (Forman et al., 2007), consistent with the accretion power one would derive if the black hole were accreting at the Bondi accretion rate, thus making M87 a jet-dominated

\footnotetext{
${ }^{12}$ Much of this radiation may actually be in the form of X-rays from the unresolved base of the jets itself
} 

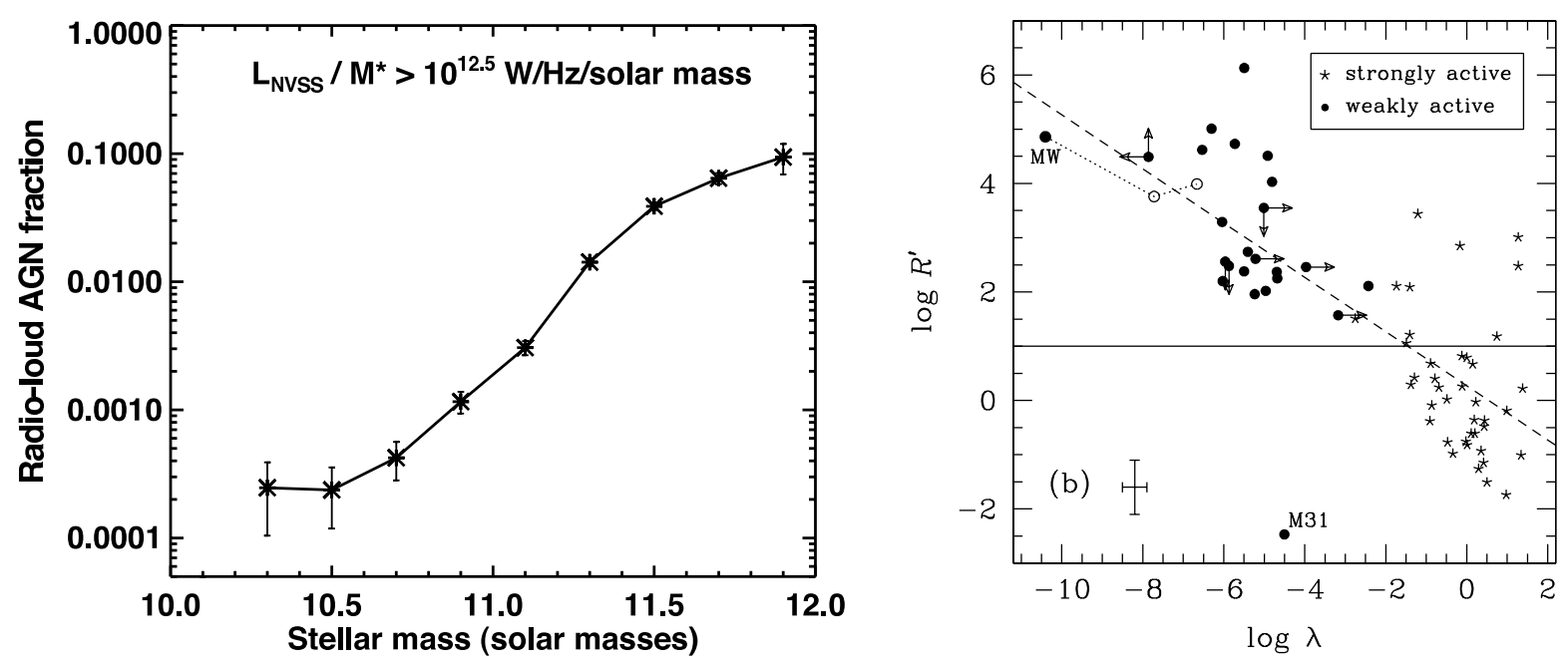

Figure 30: Left: Fraction of radio loud objects (defined as objects with a radio luminosity larger than $10^{12.5} \mathrm{~W} \mathrm{~Hz}^{-1}$ per solar mass of stars of its host galaxy) plotted against stellar mass of the host, adopted from Best et al. (2005); right: Radio loudness $R^{\prime}$ (here defined as $5 \mathrm{GHz}$ radio flux to $2500 \AA$ flux) as a function of Eddington ratio $\lambda$ (defined as the ratio of bolometric AGN flux to Eddington flux), adopted from $\mathrm{Ho}$ (2002).

low-luminosity black hole.

While a direct translation of radio loudness into feedback efficiency is challenging, this result suggests that black hole feedback might be more prevalent than just in the most powerful AGN in the most massive galaxies. In fact, the ubiquity of low-luminosity AGN, and their increased dominance at low redshift (see Fig. 8) supports both the idea of a radio maintenance mode of ongoing feedback in early type galaxies (Croton et al., 2006), as well as the picture of gentle feedback (through buoyancy rather than shocks) not just in clusters but also in galaxies.

Additionally, ongoing low-level jet activity seems much better suited at targeting gas within the galaxy than explosive outbursts in the form of powerful radio galaxies that would transport most of the energy far beyond galactic scales, and the prominent examples of radio mode feedback shown in Fig. 29] should be considered extreme outliers from more typical radio-mode feedback. This statement can, in fact, be quantified based on the global AGN evolutionary models outlined in chapter 3.1.1, which we shall discuss next.

\subsection{Quantifying the efficiency of the radio mode}

The observed omni-presence of radio core 13 in low luminosity AGN and the observed increase in radio loudness of X-ray binaries at low luminosities can be placed on a solid theoretical footing. Jets launch in the innermost regions of accretion flows around black holes, and at low luminosities, these flows likely become mechanically (i.e., advectively) cooled.

\footnotetext{
${ }^{13}$ The "core" of a jet is the brightest innermost region of the jet, where the jet just becomes optically thin to synchrotron self absorption, i.e., the synchrotron photosphere of the jet.
} 
Such flows can, to lowest order, be assumed to be scale invariant: a low luminosity accretion flow around a 10 solar mass black hole, accreting at a fixed, small fraction of the Eddington accretion rate, will be a simple, scaled down version of the same flow around a billion solar mass black hole (with the spatial and temporal scales shrunk by the mass ratio). It follows, then, that jet formation in such a flow should be similarly scale invariant.

This assumption is sufficient to derive a very generic relation between the radio luminosity emitted by such a scale invariant jet and the total (kinetic and electromagnetic) power carried down the jet, independent of the unknown details of how jets are launched and collimated (Heinz \& Sunyaev, 2003): The synchrotron radio luminosity $L_{\nu}$ of a self-absorbed jet core depends on the jet power $P_{\text {jet }}$ through

$$
L_{\text {radio }} \propto P_{\text {jet }}^{\frac{17+8 \alpha}{12}} M^{-\alpha} \sim P^{\frac{17}{12}}
$$

where $M$ is the mass of the black hole and $\alpha \sim 0$ is the observable, typically flat radio spectral index of the synchrotron power-law emitted by the core of the jet. This relation is a result of the fact that the synchrotron photosphere (the location where the jet core radiates most of its energy) moves further out as the size scale and the pressure and field strength inside the jet increase (corresponding to an increase in jet power). As the size of the photosphere increases, so does the emission. The details of the power-law relationship are an expression of the properties of synchrotron emission.

For a given black hole, the jet power should depend on the accretion rate as $P_{\text {jet }} \propto \dot{M}$ (this assumption is implicit in the assumed scale invariance). On the other hand, the emission from optically thin low luminosity accretion flows itself depends non-linearly on the accretion rate, roughly as $L_{\text {acc }} \propto \dot{M}^{2}$, since two body processes like bremsstrahlung and inverse Compton scattering dominate, which depend on the square of the density. Thus, at low accretion rates, $L_{\text {radio }} \sim L_{\text {bol }}^{\frac{17}{24}}$, which implies that black holes should become more radio loud at lower luminosities (Heinz \& Sunvaev, 2003; Merloni et al., 2003; Falcke et al., 2004). It also implies that more massive black holes should be relatively more radio loud than less massive ones, at the same relative accretion rate $\dot{M} / M$.

Equation 23 is a relation between the observable core radio flux and the underlying jet power. Once calibrated using a sample of radio sources with known jet powers, it can be used to estimate the jet power of other sources based on their radio properties alone (with appropriate provisions to account, statistically, for differences in Doppler boosting between different sources).

The cluster radio sources shown in Fig. 31 provide such a sample. Plotting the core (unresolved) radio power against the jet power inferred from cavity and shock analysis shows a clear non-linear relation between the two variables (Merloni \& Heinz, 2007). Fitting this relation provides the required constant of proportionality and is consistent (within the uncertainties) with the power-law slope of $17 / 12$ predicted by eq. (23)

$$
P_{\text {jet }}=P_{0}\left(\frac{L_{\text {radio }}}{L_{0}}\right)^{\zeta} \sim 1.6 \times 10^{36} \operatorname{ergs~s}^{-1}\left(\frac{L_{\text {radio }}}{10^{30} \mathrm{ergs} \mathrm{s}^{-1}}\right)^{0.81}
$$

with an uncertainty in the slope $\zeta$ of 0.11 , where $L_{\text {radio }}=\nu L_{\nu}$ is measured at $\nu=5 G H z$.

Because this relation was derived for the cores of jets, which display the characteristic flat self-absorbed synchrotron spectrum, care has to be taken when applying it to a sample of objects: 
only the core emission should be taken into account, while extended emission should be excluded. As discussed in 2.1.1 radio luminosity functions are separated spectrally into flat and steep sources, and we can use both samples to limit the contribution of flat spectrum sources from both ends.

Given a radio luminosity function $\Phi_{\text {rad }}$ and an appropriate correction for relativistic boosting, eq. 24 can be used to derive the kinetic luminosity function of jets (Heinz et al., 2007; Merloni \& Heinz, 2008):

$$
\Phi_{\text {kin }}\left(P_{\text {jet }}\right)=\Phi_{\text {rad }}\left[L_{0}\left(\frac{P_{\text {jet }}}{P_{0}}\right)^{\frac{1}{\zeta}}\right] \frac{1}{\zeta} \frac{L_{0}}{P_{0}}\left(\frac{P_{\text {jet }}}{P_{0}}\right)^{\frac{1-\zeta}{\zeta}}
$$

The resulting kinetic luminosity functions for the flat spectrum radio luminosity function 14 from Dunlop \& Peacock (1990) and de Zotti et al. (2005) are plotted in the right panel of Fig. 31 .

Since the figure plots $P \cdot \Phi_{\mathrm{P}}$, the curves show directly the total contribution of AGN at a given jet power to the total feedback power at a given redshift. At the low luminosity end, these curves are roughly flat, implying that low luminosity source contributed a significant fraction of the total power. These are the low-luminosity AGN presumably responsible for radio mode feedback, and they dominate the total jet power output at low redshift.

Integrating the luminosity function over $P_{\text {jet }}$ gives the local jet power density $\rho_{\text {Pjet }}$, which, at redshift zero, is of the order of $\left\langle\rho_{\text {Pjet }}\right\rangle \sim 6 \times 10^{39} \mathrm{ergs} \mathrm{s}^{-1} \mathrm{Mpc}^{-3}$, which is comparable to the local power density from supernovae, but will be significantly above the supernova power in early type galaxies (which harbor massive black holes prone to accrete in the radio mode but no young stars and thus no type 2 supernovae).

Finally, integrating $\Phi_{\text {kin }}$ over redshift gives the total kinetic energy density $u_{\text {Pjet }}$ released by jets over the history of the universe, $u_{\text {Pjet }} \sim 3 \times 10^{57} \mathrm{ergs} \mathrm{Mpc}^{-3}$. By comparing this to the local black hole mass density $\rho_{\mathrm{BH}}$ we can derive the average conversion efficiency $\eta_{\text {jet }}$ of accreted black hole mass to jet power:

$$
\eta \equiv \frac{u_{\text {Pjet }}}{\rho_{\mathrm{BH}} c^{2}} \approx 0.2 \%-0.5 \%
$$

In other words, about half a percent of the accreted black hole rest mass energy gets converted to jets, averaged over the growth history of the black hole.

Since most black hole mass was accreted during the quasar epoch, when black holes were mostly radio quiet, about $90 \%$ of the mass of a given black hole was accreted at zero efficiency (assuming that only $10 \%$ of quasars are radio loud). Thus, the average jet production efficiency during radio loud accretion must be at least a factor of 10 higher, about $2 \%-5 \%$, comparable to the radiative efficiency of quasars. These are exactly the kinds of efficiencies needed for radio mode feedback to work.

\subsection{Quasar mode feedback}

Arguments for black hole feedback on galactic scales stem primarily from three facts:

- The deviation of the bright end of the galaxy luminosity function from self-similar predictions (Springel et al., 2005), that is, a dearth of bright galaxies.

\footnotetext{
${ }^{14}$ Comparison to the steep spectrum luminosity function shows that the error in $\Phi_{P}$ from the sources missed under the steep spectrum luminosity function is at most a factor of two
} 

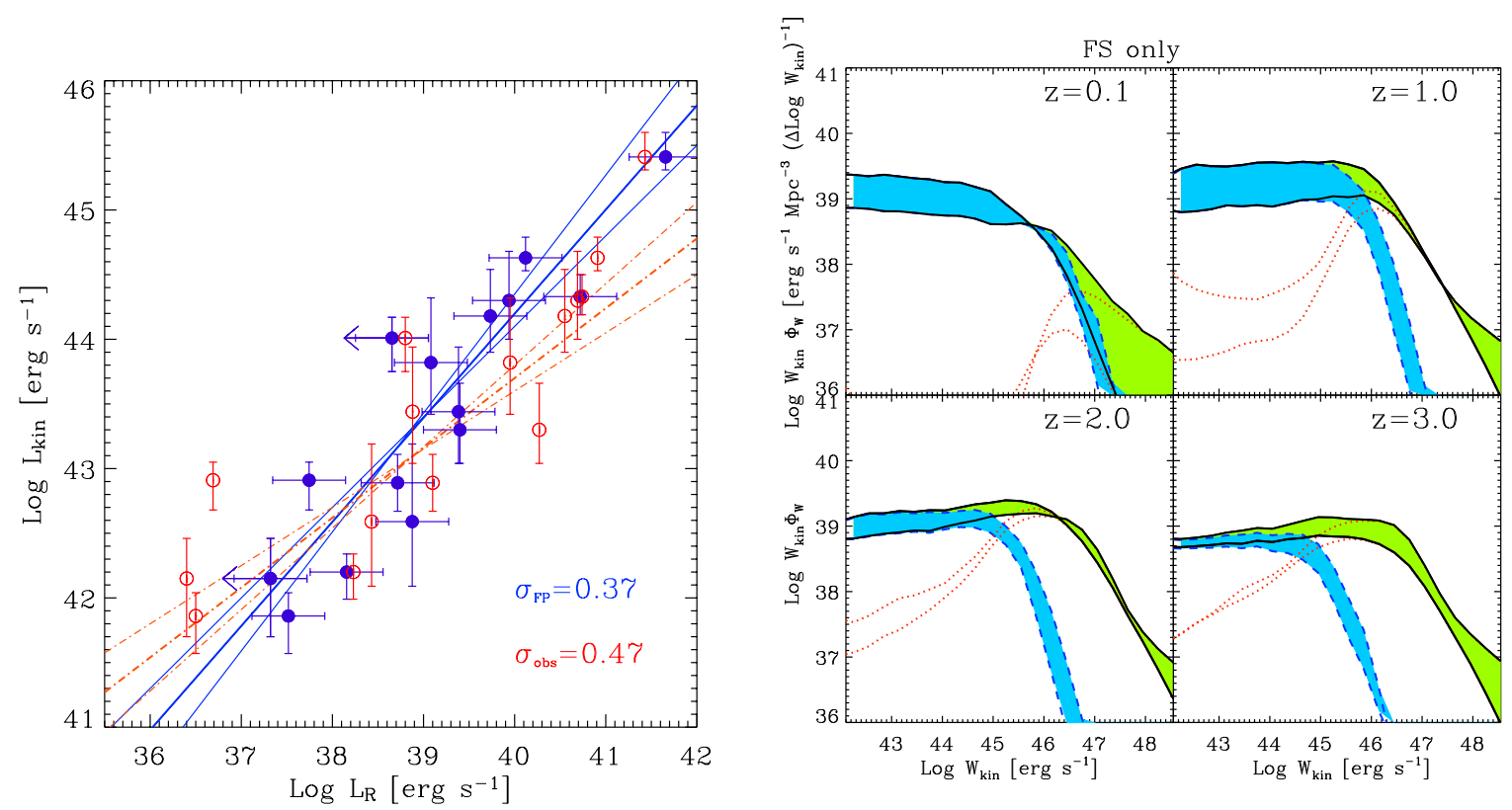

Figure 31: Left: Red open circles show jet power (measured from X-ray cavities) plotted against 5 $\mathrm{GHz}$ core radio luminosity (also shown in blue solid circles is a Doppler boosting corrected version of the same points) along the power-law fit given in eq. (24); adopted from Merloni \& Heinz (2007); right: Kinetic jet luminosity function derived from flat spectrum (FS) radio galaxy surveys, for different redshift bins (green curve shows all radio sources, blue shows radio sources in the radio mode, red shows radio sources in quasar mode); adopted from Merloni \& Heinz (2008).

- The bimodality of the galaxy distribution in color-magnitude space (Strateva et al., 2001), with early type galaxies forming the "red sequence" and late type galaxies forming the "blue cloud". Between these two populations lies, naturally, the so-called "green valley", which is relatively sparsely populated. This bimodality is shown in Fig. 32 .

- The tight relation between stellar bulge mass and black hole mass, which suggests a common formation scenario. Given that massive black holes grew mostly as quasars during the epoch of star formation, this suggests a relationship between both. In fact, the argument for quasar mode feedback was first made in part to motivate black hole - galaxy scaling relations (e.g. Silk \& Rees, 1998; Wyithe \& Loeb, 2003).

Rapidly growing black holes are attractive as agents of feedback on ongoing star formation because they have similar growth histories, they can be found in the centers of galactic bulges (the stellar populations their feedback is supposed to influence), they can release large amounts of energy isotropically, and they are likely to be fueled rapidly in response to galaxy mergers, which also trigger star formation.

Numerical simulations of black hole feedback in individual galaxy mergers have produced impressive visualizations of how rapid, isotropic energy injection by a growing black hole can heat 
and disperse the cool, star forming gas, in essence explosively terminating star formation and black hole growth (Di Matteo et al., 2005). In part as a result of these successes, quasar mode feedback is now routinely incorporated into cosmological simulations of structure formation and semi analytic models of galaxy formation (Croton et al., 2006; Bower et al., 2006).

In these simulations the prescription of how black holes accrete is simplified to variations of the Bondi accretion rate, necessitated by the unresolvably vast dynamic range of the problem. Energy is injected isotropically, which is an appropriate zero order choice given our lack of knowledge about the actual channel through which the energy is delivered. What the simulations tell us is that efficient black hole feedback can regulate star formation and black hole growth. But because black hole feedback and supernova feedback are operationally very similar, and because the presumed AGN feedback mechanism is generic, it is difficult to extract more detailed information about the AGNs themselves from the models.

In addition, the causality of the interaction of black holes with the star forming gas surrounding them is not yet fully established. It is also plausible that star formation itself provides the feedback through supernovae, and that competitive accretion starves both black holes and stars, leading to a passive link between black holes and stars.

Identifying currently ongoing episodes of feedback has proven to be difficult, in part because of the large degrees of visible- and soft X-ray extinction towards star forming regions and because of the small angular scales involved. Proving the causal connection between AGN activity and terminated star formation is even more difficult.

Generally, one would assume that galaxies caught in the act of feedback should just start their transition from the blue cloud to the red sequence, as the population of recently formed early stars fades without any replenishment. The relative under-density of galaxies in the green valley suggests that this transition is a relatively rapid process (one would expect it to occur roughly on A-star life times).

Stellar population modeling has successfully been used to identify such post-starburst galaxies. And indeed, sources have been found among this class that show clear evidence for very fast outflows in excess of $1000 \mathrm{~km} \mathrm{~s}^{-1}$ (Tremonti et al., 2007) that might be the smoking gun. Estimating the mass in the outflow has proven to be difficult, and we have to await deep imaging that can directly resolve the outflow to quantify the energetic impact of the AGN on the galactic gas.

In addition, surveys of (hard) X-ray selected AGN find these sources to preferentially lie in the green valley (from the all-sky Swift-BAT survey; Schawinski et al., 2009), as can be seen in Fig. 32. Since AGN accretion time scales can be expected to be shorter than the transition time across the green valley, this observation suggests that the AGN activity comes after star formation has been terminated. Since this is true also for hard X-ray selected AGN, this conclusion should not be affected by obscuration unless AGN in the act of feedback are so heavily obscured that even Swift cannot detect them.

Thus, the question of what is at the heart of the putative quasar mode feedback is left unanswered. Generally, AGN can release energy via three channels: Through radiation, through jets, and through un-collimated outflows (i.e., "winds").

The most obvious source of feedback energy in efficiently accreting black holes is, of course, the radiation itself. Since most bright AGN are obscured, we can infer that a high fraction of the initially emitted light is reabsorbed by the surrounding gas. If some of the energy is deposited on 


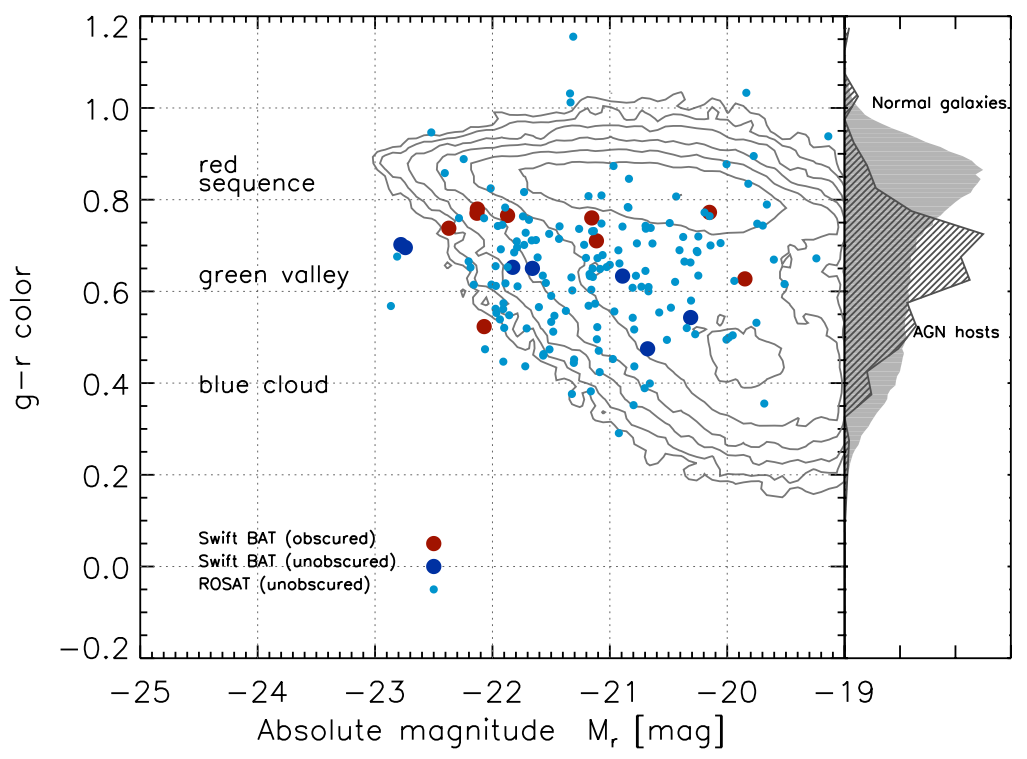

Figure 32: Contours of galaxy counts as a function of R-band magnitude and g-r color, showing the existence of two separate populations - the red sequence on top and the blue cloud on the bottom. Over-plotted are the locations of X-ray selected AGN (hard and soft), with a clear preference for a location in the "green valley" in between both populations; adopted from Schawinski et al. (2009).

sufficiently large scales (rather than into gas bound to the black hole), it can in principle supply the energy for feedback. Models of radiative feedback (Ciotti \& Ostriker, 2001; Sazonov et al., 2005) generally rely on Compton heating. The efficiency of radiative feedback requires about $10 \%$ of the radiation to be absorbed on scales outside of the Bondi radius but within the star forming region of the host. Whether radiative transfer will always conspire to provide such an arrangement is an open question.

Efficiently accreting AGN (quasars and Seyferts) are also known to generate massive winds: Optical absorption line studies show outflows at velocities of thousands of kilometers per second. The most dramatic demonstration comes from the class of broad absorption line quasars (BAL QSOs), which show high column densities of absorption in visible and X-rays (indicating high mass fluxes) and wind velocities up to $50,000 \mathrm{~km} \mathrm{~s}^{-1}$.

If column density measurements made in the X-ray band trace the same gas that produces the large outflow velocities in optical absorption lines, the power and mass contained in these winds would be of the same order as the total radiative power of these objects (Furlanetto \& Loeb, 2001). Driving such a wind would presumably require some form of mechanical input (for example, from magneto-centrifugal launching as described in chapter "Active Galactic Nuclei" by E. Perlman in this same volume) in addition to radiative driving.

The ubiquity of outflow signatures in efficiently accreting AGN, coupled with the large wind efficiencies inferred from the more extreme cases, have made AGN winds the primary mechanism invoked in feedback models (see e.g. King, 2005). Given the uncertainties in column density of 
the high-velocity gas, a direct imaging detection of outflow signatures (like the cavities in the case of AGN feedback in clusters) would provide more certainty that winds can affect the surrounding gas on the scales needed for feedback to operate.

The uncertainty about whether winds are powerful enough to drive feedback raises the interesting question whether episodes of powerful jet activity in quasars can lead to feedback on galactic scales, and whether they can be observed. In the simple framework of radio source dynamics laid out in 4.2.1 an episode of jet activity will inflate a supersonically expanding cocoon, the size scale of which depends on the jet power and the density of the environment.

In the dense environments of star forming regions, one might thus expect sources to go through a compressed evolution, with slowed or even stalled expansion as sources run into dense gas. In such a scenario, the initial expansion might produce strong shocks (given the cold gas they encounter) but at much reduced shock temperatures given the slower expansion. Is it possible that powerful radio sources in dense environments can heat the gas sufficiently to provide the quasar mode feedback postulated by semi-analytic models?

Given that about $10 \%$ of all powerful quasars are radio loud, and given that the required average feedback efficiency for the quasar mode can be an order of magnitude lower still, jet powered feedback may actually contribute significantly to the quasar mode as well. In fact, a class of sources that might represent these powerful radio quasars in the act of feedback exists in the so-called Compact-Steep-Spectrum (CSS) and the Gigahertz-Peaked-Spectrum (GPS) sources (O’Dea, 1998).

These are small scale radio sources that show clear signs of strong absorption to the radio spectrum (indicating a high local pressure and thus ISM density) and otherwise appear similar to classical radio sources but on smaller scales. The cause for their compactness has been debated since their discovery: They might be young sources, in the very early stages of supersonic expansion, or frustrated older sources, caught in very dense environments. In either case, this would be a population of sources directly heating the dense gas in the centers of galaxies, where quasar mode feedback is observed.

Recent evidence does suggest that these sources are indeed young, and that we are looking at infant powerful radio sources (e.g. Holt et al., 2008; Kunert-Bajraszewska et al., 2010). The high rate of incidence, compared to bona fide quasars, suggests that they are a short-lived phenomenon, which would make them effective short-cycle thermostats in a feedback scenario.

The detection of compact radio sources in high-redshift star forming environments seems to support the role of jets in quasar mode feedback: A number of high-power compact radio sources have been found in actively star forming regions with powerful outflows (Nesvadba et al., 2007, 2011) and in dense, high-z cooling flow environments (Siemiginowska et al., 2010). Because we know jet feedback works in the context of clusters and likely in the "maintenance" mode of feedback, and because we know that CSS and GPS sources are (a) frequent and (b) powerful, they present an attractive alternative to the wind-driven QSO mode of feedback.

Simulations of jets in dense, multi-phase environments, as might be expected in star forming galaxies, already show significant promise in solving the question of how bipolar, highly collimated jets in even very powerful radio sources could efficiently heat the gas in galaxies (Wagner \& Bicknell, 2011). 


\section{Cosmogony}

We have seen in section 3.1 that the total mass density estimated in relic supermassive black holes at $z \sim 0$ is consistent with the total mass accreted by growing black holes during (obscured and un-obscured) AGN phases for a radiative efficiency of the accretion process $\left(0.06<\epsilon_{\mathrm{rad}}<0.2\right.$, depending on the bolometric corrections and local mass density exact estimate), well in line with the prediction of classical relativistic accretion disc models (Novikov \& Thorne, 1973).

In fact, the validation of the Soltan (1982) argument implies that the last few e-folds of a SMBH's mass are mainly grown via (radiatively efficient) classical accretion discs, rather than through mergers or radiatively inefficient accretion. If this is true, however, the very process of cosmological black hole growth through accretion quickly erases the initial condition, namely, the primordial mass function of seed black holes, making it almost impossible to deduce the physical properties of early black hole formation from observations probing redshifts smaller than that corresponding to the most efficient growth $(z \approx 2-3)$. That is, unless a specific range of $\mathrm{BH}$ masses is identified which is less affected by the complex process of AGN activation during structure formation.

Indeed, some have argued that small mass black holes in isolated, small mass galaxies could have maintained a 'memory' of the seeding mechanism (in their location with respect to the scaling relations defined for more massive systems, for example), being less affected by the multiple generations of hierarchical mergers in the emerging cosmic web (see e.g. Volonteri 2010). Very few observational constraints are available for this class of objects, however.

On the other hand, the observation of luminous quasars at $z \simeq 6$ (e.g. Fan et al., 2001) has shown that it is possible to probe directly the earlier epochs of massive black hole assembly, and thus to try to directly constrain the various physical processes responsible for planting the seeds that grow into the giant monsters in the nuclei of galaxies.

\subsection{The first black holes: observational constraints and theoretical ideas}

The observed luminosity functions of AGN suggest a rapid decline of the total luminosity density above $z \approx 3$ (see Figure 18). At face value, the constraints on the very high redshift evolution of the population at $z>5$ come primarily from bright optical quasars detected in very large area surveys, but recent indications from large area and moderately deep radio (Wall et al., 2005) and X-ray (Civano et al., 2011) surveys do provide a consistent picture for the evolution of the most luminous AGN over all observational wavebands (Figure 33).

The observed rapid decline of QSO number density towards high redshift translates into a rapid decrease in the number of AGN-generated ionizing photons towards the end of the reionization epoch. Accurate determination of the QSO rest-frame UV luminosity function are thus crucial to assess the role of growing black hole might have played in re-ionizing the universe. Current estimates suggest that galaxies do dominate the comoving emissivity of ionizing photons escaping in the inter-galactic medium at $z>4$ (Haardt \& Madau, 2012, and references therein).

Despite their rarity, very high-redshift QSOs can provide interesting constraints on the early evolution (and even formation mechanisms) of nuclear supermassive black holes. The high metal enrichment observed in high-z AGNs (see section 1.1), even in those close to reionization $(z \sim 6)$, indicate that the host galaxies of these AGNs must have undergone a powerful and rapid burst of star formation. And indeed, vigorous star formation is observed in such high-z 

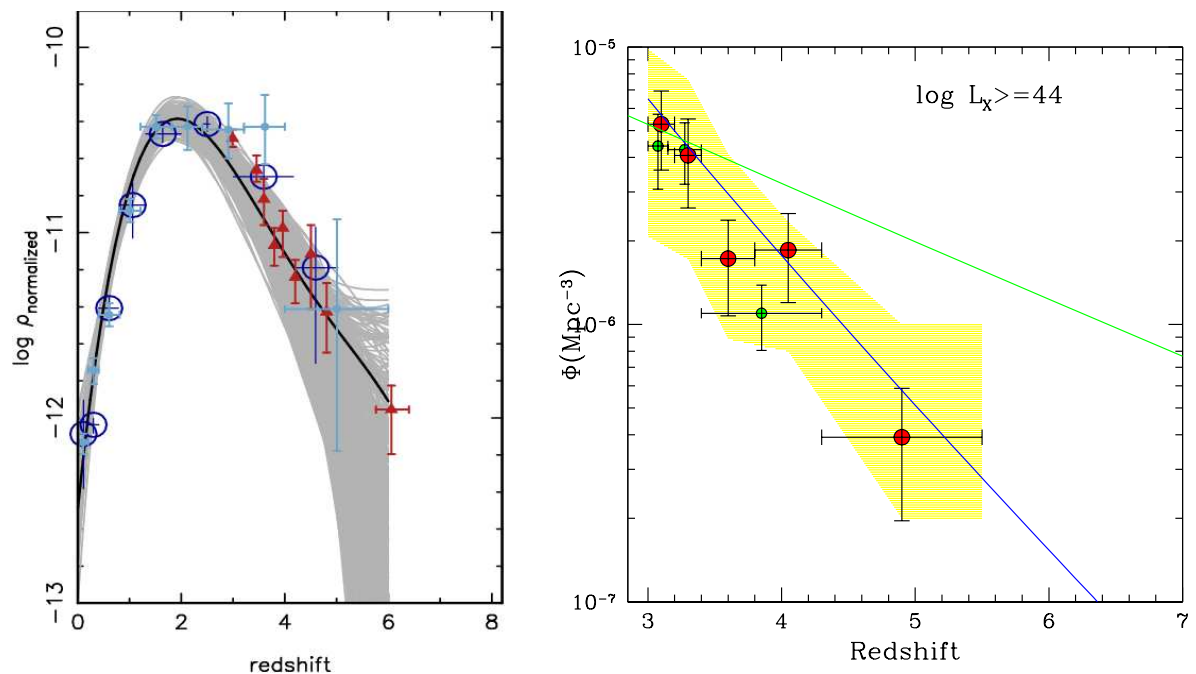

Figure 33: Observational constraints on the decline of QSO number densities at high redshift. Left: Relative space density of QSOs $(\rho)$ as a function of redshift. The shaded area and black line represent the current QSO space-density determination from the Parkes quarter-Jansky flat-spectrum sample of radio selected QSOs. Light blue filled and dark blue open circles show the soft X-ray data from ROSAT, Chandra and XMM-Newton surveys. Space density behavior of optically-selected QSOs is given by the set of dark red triangles. The X-ray and optical QSO data were scaled vertically to match the current determination of space density at redshifts 2 to 2.5. From Wall et al. (2005). Right: The comoving space density at bright $2-10 \mathrm{keV} \mathrm{X-}$ ray luminosity from the Chandra-COSMOS survey, computed taking into account the effect of obscuration. The blue curve corresponds to the Xray selected AGN space density computed for the same luminosity limit from models of the CXRB. The yellow shaded area represents the maximum and minimum space density. The green symbols correspond to the data of XMMCOSMOS. From Civano et al. (2011). 
quasars, as inferred from the detection of prominent PAH (Polycyclic Aromatic Hydrocarbons) features, strong far-IR emission and from the detection of the [CII]158m line (see Maiolino, 2009), redshifted to millimeter wavelengths. Most likely, we are witnessing the coeval, rapid formation of massive bulges along with their supermassive central black holes.

These luminous quasars detected at $z>6$, when the universe was less than 1 Gyr old have estimated BH masses (from the "virial method") in excess of $\approx 10^{9} M_{\odot}$, and it is by no means a trivial task to grow such massive holes in the relatively short time available. Assuming continuous growth at an Eddington ratio of $\lambda=L / L_{\mathrm{Edd}}$ :

$$
\frac{d M_{\mathrm{BH}}}{d t}=\left(1-\epsilon_{\mathrm{rad}}\right) \lambda \times L_{\mathrm{Edd}} /\left(\epsilon_{\mathrm{rad}} c^{2}\right)
$$

we have, for the final $\mathrm{BH}$ mass as a function of the initial mass

$$
M_{\mathrm{BH}, \mathrm{f}}(t)=M_{i} \exp \left(1-\epsilon_{\mathrm{rad}}\right) / \epsilon_{\mathrm{rad}} \times\left(t / \tau_{\mathrm{salp}}\right),
$$

where we have defined the typical e-folding time (the so-called "Salpeter time") as

$$
\tau_{\text {salp }}=\frac{\lambda c \sigma_{\mathrm{T}}}{4 \pi G m_{\mathrm{p}}}=0.45\left(\frac{1}{\lambda}\right) \mathrm{Gyr}
$$

Depending on the redshift of formation of the seed of mass $M_{i}$, and on the average radiative efficiency of the accretion process, only a limited range of final $\mathrm{BH}$ masses can be reached at $z=6$, as shown in Figure 34 (Shapiro, 2005). If the BH seed masses are in the range expected from Pop III remnants, of the order of a few hundred solar masses, then highly radiative efficient $\left(\epsilon_{\mathrm{rad}}>0.1\right)$ accretion is excluded, as it would not allow enough mass to be accumulated into the black hole rapidly enough.

Such a scenario has a number of major difficulties. First of all, if the accretion is indeed continuous and proceeds at high Eddington rates, the accretion flow should form a geometrically thin, optically thick disk. If this is the case, even a black hole with zero initial dimensionless angular momentum $a_{i}=0$ will be rapidly spun-up by the angular momentum captured with the accreting gas. Bardeen (1970) has shown that in this case the final spin obeys:

$$
a_{f}=\frac{r_{\mathrm{ms}, \mathrm{i}}^{1 / 2}}{3} \frac{M_{i}}{M_{f}}\left[4-\sqrt{3 r_{\mathrm{ms}, \mathrm{i}}\left(\frac{M_{i}}{M_{f}}\right)^{2}-2}\right]
$$

where $r_{\mathrm{ms}, \mathrm{i}}$ is the radius of the marginally stable orbit of the initial black hole (itself a monotonic function of spin, with $r_{\mathrm{ms}}(a=0)=6$ and $r_{\mathrm{ms}}(a=1)=1$ in gravitational units). Thus, an initially non-spinning $\mathrm{BH}$ is spun up by accretion of gas in a classical geometrically thin and optically thick accretion disc as soon a: $15 M_{f} / M_{i}=\sqrt{6}$. Since an accretion disc around a maximally rotating hole will radiate with an efficiency $\epsilon_{\mathrm{rad}}=0.42$, we are left with the impossibility of growing black holes larger than a few thousands of solar masses via prolonged coherent accretion onto a stellar mass seed (see Figure 34).

A few solutions have been proposed to the above problem.

\footnotetext{
${ }^{15}$ Note that the above calculation assumes that there is no torque at the inner boundary of the accretion disc (Novikov \& Thorne, 1973). Magnetic linkage between the disc, the plunging region and the event horizon can modify the above picture, reducing the maximal spin a BH can reach (Krolik et al., 2005). Nonetheless, most numerical models of geometrically thin magnetized discs are still consistent with a rapid spin of the $\mathrm{BH}$.
} 


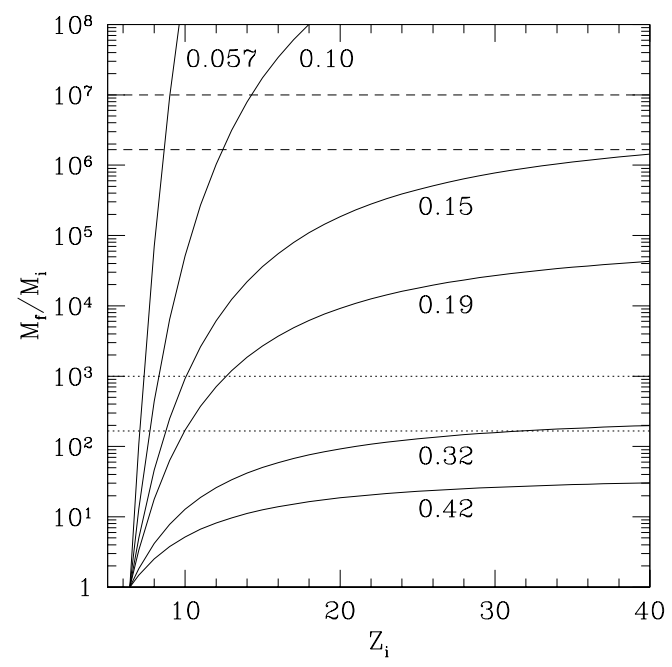

Figure 34: Ratio of final-to-initial black hole mass at $z=6$ calculated from eq. (28) assuming $\lambda=1$ (i.e. continuous Eddington-limited accretion) as a function of the formation redshift of the seed black hole. Each solid curve is labeled with the corresponding value of the radiative efficiency $\epsilon_{\mathrm{rad}}$. Dotted horizontal lines show the ratio implied by the observed $z \sim 6$ QSOs for massive seeds, while the dashed ones that for stellar mass (Pop III; see section 5.1 for details). From Shapiro (2005).

1. First, accretion might not be coherent, but rather stochastic, such that the accreting gas comes into the gravitational sphere of influence of the black hole in parcels with randomly oriented angular momenta. If the mass of each parcel is small enough (roughly speaking, if $\Delta M \ll \sqrt{6} M_{i}$ ), then the $\mathrm{BH}$ spin vector performs a random walk, but as it is easier to spin a black hole down than up, the net effect is a relatively low average spin (and correspondingly lower radiative efficiency) of the final hole (King \& Pringle, 2006).

2. In the primordial cosmological setup where proto-galaxies form, the first black holes grow in a very gas rich environment, and there is no reason to believe that the gas could not flow towards the central black holes at vastly super-Eddington rates. It is not clear, however, whether the hole can swallow matter that fast. On the one hand, quasi-spherical inflows can be established, where the radial velocity of the accreting gas is so high that the photons produced inside the disc by the viscous torques cannot escape (Frank et al., 2002). In those cases, although the emerging luminosity is at most logarithmically in excess of the Eddington limit, the accretion rate onto the hole can be orders of magnitude larger. On the other hand, the accretion flow can start blowing out matter at the (large) radius where the locally produced energy exceeds the local Eddington limit (Shakura \& Sunvaev, 1973). A powerful wind ensues, which may prevent the mass accretion rate onto the black hole from exceeding the Eddington limit by more than a factor of a few.

3. Black hole seeds in the early universe can be more massive than the remnants of Pop III stars. These would have formed by direct collapse of primordial massive stars (see 
e.g. Volonteri, 2010). For example, some theoretical models have argued that the gas chemistry in the most massive, hottest primordial DM halos can prevent fragmentation of the cooling gas and lead to the formation of very massive stars. Their cores will rapidly collapse, leaving a black hole at the center of a quasi-static, radiation pressure-supported supermassive star. The resulting object, called a quasistar, resembles a red giant with a luminosity comparable to a Seyfert nucleus. The black hole grows inside it until the cooling photosphere can no-longer sustain its own radiation pressure and the envelope disperses, leaving behind the naked seed black hole of typically $10^{4}-10^{5} M_{\odot}$ (Begelman, 2010).

4. Finally, a large number of major mergers could help relax the demands on the efficiency and stability of gas accretion on the first black holes by enhancing the final-to-initial mass ratio by a factor of the order of the number of equal-mass (or major) mergers along the main tree of the hierarchy.

Cosmological numerical simulations (Li et al., 2007) provide a possible route to the formation of a $10^{9} M_{\odot}$ at $z \sim 6$ by starting with Pop III stellar mass seeds at $z \sim 30$ which experience an early phase of continuous, Eddington-limited accretion (subject, however, to the limitations discussed above), before entering the merger tree at $z \sim 14$, where a large number of major merger events is able to accumulate the final mass, even in the presence of AGN feedback.

Analytic and semi-analytic models of the early assembly of massive black holes that include (with varying degrees of sophistication) the many competing processes (mergers, gravitational wave recoil and nuclear black hole ejection, dynamical friction on wandering BHs, pristine gas accretion, etc.) make clear predictions for the early seed mass distributions, the initial conditions for SMBH growth that we would like to probe. Figure 35 shows one such prediction, comparing the outcomes of three different formation scenarios: direct collapse, runaway stellar mergers in high-redshift stellar clusters, and Population III remnants. A more complete census of the AGN population at $z \sim 6$ or even a few detections of $z \sim 10$ AGN with the next generation of large astronomical facilities could provide direct means to distinguish among these simple formation scenarios, allowing us to glimpse into the obscured epoch when the first nuclear black holes formed.

\section{Acknowledgments}

We would like to thank warmely our Editor William C. Keele for the kind invitation to write this chapter in the new edition of the "Planets, Stars and Stellar System" book, and for the patience and endurance he demonstrated in putting up with our delays. Obviously, this work could not have been possible without the many contributions from a vast number of colleagues and collaborators. In particular, we whish to thank Viola Allevato, Roberto Assef, Silvia Bonoli, Niel Brandt, Marcella Brusa, Johannes Buchner, Nico Cappelluti, Scott Croom, Francesco De Gasperin, Andy Fabian, Guenther Hasinger, Phil Hopkins, Brian McNamara, Eric Perlman, Gordon Richards, Marta Volonteri. AM's work is partially supported by the DFG Cluster of Excellence "Origin and Structure of the Universe". 


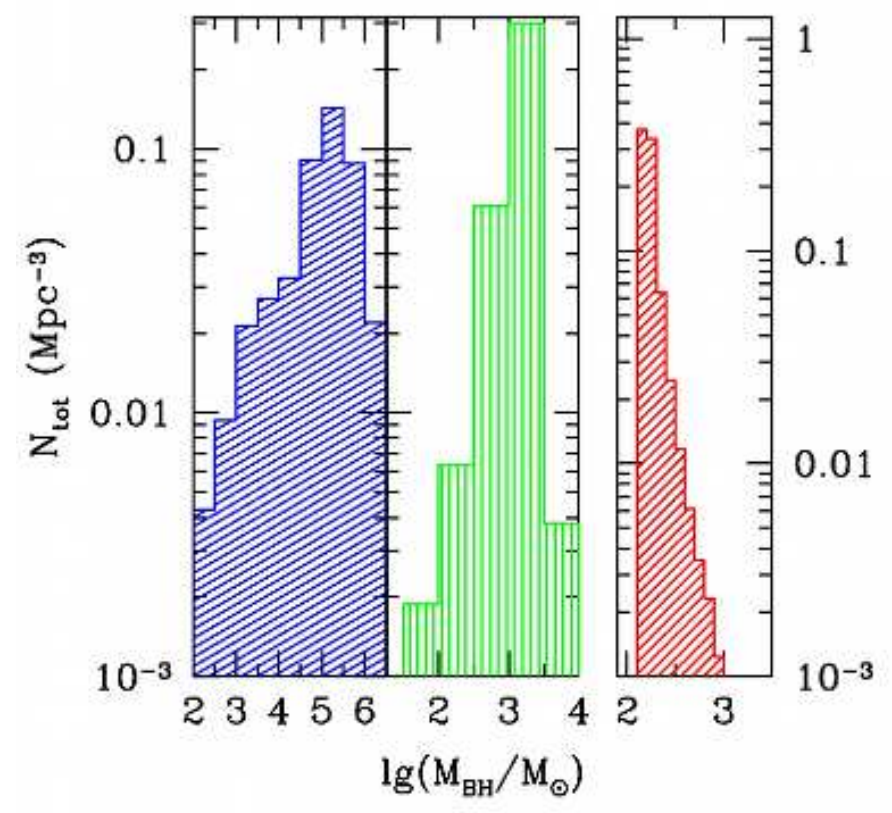

Figure 35: Mass function of seed massive Black Holes for three different formation scenarios: direct collapse (left), runaway stellar mergers in high-redshift stellar clusters (center) and Population III remnants (right). Note the different y-axis scale for the Pop III case. From (Volonteri, 2010). 


\section{Cross References: Chapters in the same book}

"Active Galactic Nuclei" by E. Perlman

"Clusters of Galaxies" by R. Bower

"Galaxies in the cosmological context" by G. De Lucia

"Large Scale Structure of the universe" by A. Coil

\section{References}

Allen, S. W., Dunn, R. J. H., Fabian, A. C., Taylor, G. B., \& Reynolds, C. S. 2006, Monthly Notices of the Royal Astronomical Society, 372, 21

Allevato, V., et al. 2011, Astrophysical Journal, 736, 99

Antonucci, R. 1993, Annual Reviews of adAstronomy and Astrophysics, 31, 473

Assef, R. J., et al. 2011, Astrophysical Journal, 728, 56

Bardeen, J. M. 1970, Astrophysical Journal, 161, 103

Begelman, M. C. 2010, Monthly Notices of the Royal Astronomical Society, 402, 673

Begelman, M. C., \& Cioffi, D. F. 1989, Astrophysical Journal Letters, 345, L21

Bennert, V. N., Auger, M. W., Treu, T., Woo, J.-H., \& Malkan, M. A. 2011, Astrophysical Journal, 742, 107

Best, P. N., Kauffmann, G., Heckman, T. M., Brinchmann, J., Charlot, S., Ivezić, Ž., \& White, S. D. M. 2005, Monthly Notices of the Royal Astronomical Society, 362, 25

Boehringer, H., Voges, W., Fabian, A. C., Edge, A. C., \& Neumann, D. M. 1993, Monthly Notices of the Royal Astronomical Society, 264, L25

Bonoli, S., Marulli, F., Springel, V., White, S. D. M., Branchini, E., \& Moscardini, L. 2009, Monthly Notices of the Royal Astronomical Society, 396, 423

Bower, R. G., Benson, A. J., Malbon, R., Helly, J. C., Frenk, C. S., Baugh, C. M., Cole, S., \& Lacey, C. G. 2006, Monthly Notices of the Royal Astronomical Society, 370, 645

Boyle, B. J., \& Terlevich, R. J. 1998, Monthly Notices of the Royal Astronomical Society, 293, L49

Branchesi, M., Gioia, I. M., Fanti, C., Fanti, R., \& Perley, R. 2006, Astronomy and Astrophysics, 446, 97

Brandt, W. N., \& Hasinger, G. 2005, Annual Reviews of adAstronomy and Astrophysics, 43, 827

Brusa, M., et al. 2010, Astrophysical Journal, 716, 348 
Burns, J. O. 1990, Astronomical Journal, 99, 14

Cappelluti, N., et al. 2009, Astronomy and Astrophysics, 497, 635

Castor, J., McCray, R., \& Weaver, R. 1975, Astrophysical Journal Letters, 200, L107

Chaudhary, P., Brusa, M., Hasinger, G., Merloni, A., \& Comastri, A. 2010, Astronomy and Astrophysics, 518, A58+

Ciotti, L., \& Ostriker, J. P. 2001, Astrophysical Journal, 551, 131

Civano, F., et al. 2011, Astrophysical Journal, 741, 91

Cole, S., et al. 2001, Monthly Notices of the Royal Astronomical Society, 326, 255

Cowie, L. L., Songaila, A., Hu, E. M., \& Cohen, J. G. 1996, Astronomical Journal, 112, 839

Croom, S. M., et al. 2009, Monthly Notices of the Royal Astronomical Society, 399, 1755

Croston, J. H., Hardcastle, M. J., \& Birkinshaw, M. 2005, Monthly Notices of the Royal Astronomical Society, 357, 279

Croton, D. J., et al. 2006, Monthly Notices of the Royal Astronomical Society, 365, 11

Danese, L., Franceschini, A., Toffolatti, L., \& de Zotti, G. 1987, Astrophysical Journal Letters, $318, \mathrm{~L} 15$

de Zotti, G., Massardi, M., Negrello, M., \& Wall, J. 2010, The Astronomy and Astrophysics Review, 18, 1

de Zotti, G., Ricci, R., Mesa, D., Silva, L., Mazzotta, P., Toffolatti, L., \& González-Nuevo, J. 2005, Astronomy and Astrophysics, 431, 893

Di Matteo, T., Springel, V., \& Hernquist, L. 2005, Nature, 433, 604

Dong, R., Rasmussen, J., \& Mulchaey, J. S. 2010, Astrophysical Journal, 712, 883

Dunlop, J. S., \& Peacock, J. A. 1990, Monthly Notices of the Royal Astronomical Society, 247, 19

Fabian, A. C. 1994, Annual Reviews of adAstronomy and Astrophysics, 32, 277

Fabian, A. C., \& Iwasawa, K. 1999, Monthly Notices of the Royal Astronomical Society, 303, L34

Fabian, A. C., Sanders, J. S., Taylor, G. B., Allen, S. W., Crawford, C. S., Johnstone, R. M., \& Iwasawa, K. 2006, Monthly Notices of the Royal Astronomical Society, 366, 417

Falcke, H., Körding, E., \& Markoff, S. 2004, Astronomy and Astrophysics, 414, 895

Fan, X., et al. 2001, Astronomical Journal, 121, 54 
Ferrarese, L., \& Merritt, D. 2000, Astrophysical Journal Letters, 539, L9

Forman, W., et al. 2007, Astrophysical Journal, 665, 1057

Franceschini, A., Vercellone, S., \& Fabian, A. C. 1998, Monthly Notices of the Royal Astronomical Society, 297, 817

Frank, J., King, A., \& Raine, D. 2002, Accretion power in astrophysics. 3rd ed. (Cambridge, UK: Cambridge University Press. ISBN 0-521-62957-8, 2002, XIV + 384 pp.)

Fukugita, M., \& Peebles, P. J. E. 2004, Astrophysical Journal, 616, 643

Furlanetto, S. R., \& Loeb, A. 2001, Astrophysical Journal, 556, 619

Gallo, E., Fender, R. P., \& Pooley, G. G. 2003, Monthly Notices of the Royal Astronomical Society, 344, 60

Gebhardt, K., et al. 2000, Astrophysical Journal Letters, 539, L13

Giacconi, R., Gursky, H., Paolini, F. R., \& Rossi, B. B. 1962, Physical Review Letters, 9, 439

Gilli, R., Comastri, A., \& Hasinger, G. 2007, Astronomy and Astrophysics, 463, 79

Gültekin, K., et al. 2009, Astrophysical Journal, 698, 198

Haardt, F., \& Madau, P. 2012, Astrophysical Journal, 746, 125

Hamann, F., \& Ferland, G. 1992, Astrophysical Journal Letters, 391, L53

Häring, N., \& Rix, H. 2004, Astrophysical Journal Letters, 604, L89

Hart, Q. N., Stocke, J. T., \& Hallman, E. J. 2009, Astrophysical Journal, 705, 854

Hasinger, G. 2008, Astronomy and Astrophysics, 490, 905

Hasinger, G., Miyaji, T., \& Schmidt, M. 2005, Astronomy and Astrophysics, 441, 417

Heinz, S., Brüggen, M., Young, A., \& Levesque, E. 2006, Monthly Notices of the Royal Astronomical Society, 373, L65

Heinz, S., Merloni, A., \& Schwab, J. 2007, Astrophysical Journal Letters, 658, L9

Heinz, S., \& Sunyaev, R. A. 2003, Monthly Notices of the Royal Astronomical Society, 343, L59

Ho, L. C. 2002, Astrophysical Journal, 564, 120

—. 2008, Annual Reviews of adAstronomy and Astrophysics, 46, 475

Holt, J., Tadhunter, C. N., \& Morganti, R. 2008, Monthly Notices of the Royal Astronomical Society, 387, 639

Hopkins, P. F., Murray, N., \& Thompson, T. A. 2009, Monthly Notices of the Royal Astronomical Society, 398, 303 
Hopkins, P. F., Richards, G. T., \& Hernquist, L. 2007, Astrophysical Journal, 654, 731

Iwasawa, K., \& Taniguchi, Y. 1993, Astrophysical Journal Letters, 413, L15

Juarez, Y., Maiolino, R., Mujica, R., Pedani, M., Marinoni, S., Nagao, T., Marconi, A., \& Oliva, E. 2009, Astronomy and Astrophysics, 494, L25

King, A. 2005, Astrophysical Journal Letters, 635, L121

King, A. R., \& Pringle, J. E. 2006, Monthly Notices of the Royal Astronomical Society, 373, L90

Kirkpatrick, C. C., Gitti, M., Cavagnolo, K. W., McNamara, B. R., David, L. P., Nulsen, P. E. J., \& Wise, M. W. 2009, Astrophysical Journal Letters, 707, L69

Krolik, J. H., Hawley, J. F., \& Hirose, S. 2005, Astrophysical Journal, 622, 1008

Kunert-Bajraszewska, M., Gawroński, M. P., Labiano, A., \& Siemiginowska, A. 2010, Monthly Notices of the Royal Astronomical Society, 408, 2261

Laing, R. A., \& Bridle, A. H. 2002, Monthly Notices of the Royal Astronomical Society, 336, 328

Li, Y., et al. 2007, Astrophysical Journal, 665, 187

Longair, M. S. 1966, Nature, 211, 949

—. 2008, Galaxy Formation, ed. Longair, M. S.

Madau, P., Ferguson, H. C., Dickinson, M. E., Giavalisco, M., Steidel, C. C., \& Fruchter, A. 1996, Monthly Notices of the Royal Astronomical Society, 283, 1388

Magorrian, J., et al. 1998, Astronomical Journal, 115, 2285

Maiolino, R. 2009, in Astronomical Society of the Pacific Conference Series, Vol. 408, The Starburst-AGN Connection, ed. W. Wang, Z. Yang, Z. Luo, \& Z. Chen, 235-+

Marconi, A., Risaliti, G., Gilli, R., Hunt, L. K., Maiolino, R., \& Salvati, M. 2004, Monthly Notices of the Royal Astronomical Society, 351, 169

Martini, P., \& Weinberg, D. H. 2001, Astrophysical Journal, 547, 12

Massardi, M., Bonaldi, A., Negrello, M., Ricciardi, S., Raccanelli, A., \& de Zotti, G. 2010, Monthly Notices of the Royal Astronomical Society, 404, 532

Mateos, S., et al. 2008, Astronomy and Astrophysics, 492, 51

McNamara, B. R., \& Nulsen, P. E. J. 2007, Annual Reviews of adAstronomy and Astrophysics, 45, 117

McNamara, B. R., Nulsen, P. E. J., Wise, M. W., Rafferty, D. A., Carilli, C., Sarazin, C. L., \& Blanton, E. L. 2005, Nature, 433, 45 
McNamara, B. R., Rohanizadegan, M., \& Nulsen, P. E. J. 2011, Astrophysical Journal, 727, 39

Merloni, A., \& Heinz, S. 2007, Monthly Notices of the Royal Astronomical Society, 381, 589

—. 2008, Monthly Notices of the Royal Astronomical Society, 388, 1011

Merloni, A., Heinz, S., \& di Matteo, T. 2003, Monthly Notices of the Royal Astronomical Society, 345,1057

Merloni, A., et al. 2010, Astrophysical Journal, 708, 137

Morsony, B. J., Heinz, S., Brüggen, M., \& Ruszkowski, M. 2010, Monthly Notices of the Royal Astronomical Society, 407, 1277

Nesvadba, N. P. H., Lehnert, M. D., De Breuck, C., Gilbert, A., \& van Breugel, W. 2007, Astronomy and Astrophysics, 475, 145

Nesvadba, N. P. H., Polletta, M., Lehnert, M. D., Bergeron, J., De Breuck, C., Lagache, G., \& Omont, A. 2011, Monthly Notices of the Royal Astronomical Society, 415, 2359

Novikov, I. D., \& Thorne, K. S. 1973, in Black Holes (Les Astres Occlus), ed. C. Dewitt \& B. S. Dewitt, 343-450

Nulsen, P. E. J., Jones, C., Forman, W. R., David, L. P., McNamara, B. R., Rafferty, D. A., Bîrzan, L., \& Wise, M. W. 2007, in Heating versus Cooling in Galaxies and Clusters of Galaxies, ed. H. Böhringer, G. W. Pratt, A. Finoguenov, \& P. Schuecker , 210-+

O'Dea, C. P. 1998, PASP, 110, 493

Owen, F. N., Eilek, J. A., \& Kassim, N. E. 2000, Astrophysical Journal, 543, 611

Padovani, P., Mainieri, V., Tozzi, P., Kellermann, K. I., Fomalont, E. B., Miller, N., Rosati, P., \& Shaver, P. 2009, Astrophysical Journal, 694, 235

Perley, R. A., Dreher, J. W., \& Cowan, J. J. 1984, Astrophysical Journal Letters, 285, L35

Peterson, B. M., et al. 2004, Astrophysical Journal, 613, 682

Peterson, J. R., Kahn, S. M., Paerels, F. B. S., Kaastra, J. S., Tamura, T., Bleeker, J. A. M., Ferrigno, C., \& Jernigan, J. G. 2003, Astrophysical Journal, 590, 207

Ponman, T. J., Sanderson, A. J. R., \& Finoguenov, A. 2003, Monthly Notices of the Royal Astronomical Society, 343, 331

Puchwein, E., Sijacki, D., \& Springel, V. 2008, Astrophysical Journal Letters, 687, L53

Rafferty, D. A., McNamara, B. R., Nulsen, P. E. J., \& Wise, M. W. 2006, Astrophysical Journal, 652,216

Reynolds, C. S., Heinz, S., \& Begelman, M. C. 2001, Astrophysical Journal Letters, 549, L179

Richards, G. T., et al. 2006, Astronomical Journal, 131, 2766 
Ryle, M., \& Clarke, R. W. 1961, Monthly Notices of the Royal Astronomical Society, 122, 349

Ryle, M., \& Scheuer, P. A. G. 1955, Royal Society of London Proceedings Series A, 230, 448

Salviander, S., Shields, G. A., Gebhardt, K., \& Bonning, E. W. 2007, Astrophysical Journal, 662,131

Sandage, A. 1965, Astrophysical Journal, 141, 1560

Sazonov, S. Y., Ostriker, J. P., Ciotti, L., \& Sunyaev, R. A. 2005, Monthly Notices of the Royal Astronomical Society, 358, 168

Schawinski, K., Virani, S., Simmons, B., Urry, C. M., Treister, E., Kaviraj, S., \& Kushkuley, B. 2009, Astrophysical Journal Letters, 692, L19

Schmidt, M. 1963, Nature, 197, 1040

Schmidt, M., \& Green, R. F. 1983, Astrophysical Journal, 269, 352

Shakura, N. I., \& Sunyaev, R. A. 1973, Astronomy and Astrophysics, 24, 337

Shankar, F. 2009, New Astron. Review, 53, 57

Shapiro, S. L. 2005, Astrophysical Journal, 620, 59

Short, C. J., \& Thomas, P. A. 2009, Astrophysical Journal, 704, 915

Siemiginowska, A., Burke, D. J., Aldcroft, T. L., Worrall, D. M., Allen, S., Bechtold, J., Clarke, T., \& Cheung, C. C. 2010, Astrophysical Journal, 722, 102

Silk, J., \& Rees, M. J. 1998, Astronomy and Astrophysics, 331, L1

Simpson, C. 2005, Monthly Notices of the Royal Astronomical Society, 360, 565

Smolčić, V., et al. 2009, Astrophysical Journal, 696, 24

Soltan, A. 1982, Monthly Notices of the Royal Astronomical Society, 200, 115

Springel, V., et al. 2005, Nature, 435, 629

Steffen, A. T., Barger, A. J., Cowie, L. L., Mushotzky, R. F., \& Yang, Y. 2003, Astrophysical Journal Letters, 596, L23

Steffen, A. T., Strateva, I., Brandt, W. N., Alexander, D. M., Koekemoer, A. M., Lehmer, B. D., Schneider, D. P., \& Vignali, C. 2006, Astronomical Journal, 131, 2826

Stern, D., et al. 2005, Astrophysical Journal, 631, 163

Strateva, I., et al. 2001, Astronomical Journal, 122, 1861

Tabor, G., \& Binney, J. 1993, Monthly Notices of the Royal Astronomical Society, 263, 323

Thorne, K. S. 1994, Black holes and time warps: Einstein's outrageous legacy, ed. Thorne, K. S. 
Treister, E., Urry, C. M., \& Virani, S. 2009, Astrophysical Journal, 696, 110

Treister, E., et al. 2006, Astrophysical Journal, 640, 603

Tremonti, C. A., Moustakas, J., \& Diamond-Stanic, A. M. 2007, Astrophysical Journal Letters, 663, L77

Ueda, Y., Akiyama, M., Ohta, K., \& Miyaji, T. 2003, Astrophysical Journal, 598, 886

Urry, C. M., \& Padovani, P. 1995, PASP, 107, 803

Vernaleo, J. C., \& Reynolds, C. S. 2006, Astrophysical Journal, 645, 83

Volonteri, M. 2010, The Astronomy and Astrophysics Review, 18, 279

Wagner, A. Y., \& Bicknell, G. V. 2011, Astrophysical Journal, 728, 29

Wall, J. V., Jackson, C. A., Shaver, P. A., Hook, I. M., \& Kellermann, K. I. 2005, Astronomy and Astrophysics, 434, 133

Willott, C. J., Rawlings, S., Blundell, K. M., Lacy, M., \& Eales, S. A. 2001, Monthly Notices of the Royal Astronomical Society, 322, 536

Wise, M. W., McNamara, B. R., Nulsen, P. E. J., Houck, J. C., \& David, L. P. 2007, Astrophysical Journal, 659, 1153

Worsley, M. A., et al. 2005, Monthly Notices of the Royal Astronomical Society, 357, 1281

Wyithe, J. S. B., \& Loeb, A. 2003, Astrophysical Journal, 595, 614

Xue, Y. Q., et al. 2011, Astrophysical Journal Supplements, 195, 10

Young, M., Elvis, M., \& Risaliti, G. 2009, Astrophysical Journal Supplements, 183, 17

Yu, Q., \& Tremaine, S. 2002, Monthly Notices of the Royal Astronomical Society, 335, 965 